To appear in the Astrophysical Journal

\title{
A Deep Chandra X-ray Observation of the Embedded Young Cluster in NGC 2024
}

\author{
Stephen Skinner \\ CASA, Univ. of Colorado, Boulder, CO 80309-0389 \\ Marc Gagné and Emily Belzer \\ Dept. of Geology \&3 Astronomy, West Chester Univ., West Chester, PA 19383-2130
}

\begin{abstract}
We present results of a sensitive $76 \mathrm{ksec}$ Chandra observation of the young stellar cluster in NGC 2024, lying at a distance of $\sim 415$ pc in the Orion B giant molecular cloud. Previous infrared observations have shown that this remarkable cluster contains several hundred embedded young stars, most of which are still surrounded by circumstellar disks. Thus, it presents a rare opportunity to study $\mathrm{X}$-ray activity in a large sample of optically invisible protostars and classical $\mathrm{T}$ Tauri stars (cTTS) undergoing accretion. Chandra detected 283 X-ray sources of which 248 were identified with counterparts at other wavelengths, mostly in the near-infrared. Astrometric registration of Chandra images against the Two Micron All Sky Survey (2MASS) resulted in positional offsets of $\approx 0.25^{\prime \prime}$ near field center, yielding high confidence indentifications of infrared counterparts. The Chandra detections are characterized by hard heavily-absorbed spectra and spectacular variability. Spectral analysis of more than 100 of the brightest X-ray sources yields a mean extinction $\left\langle\mathrm{A}_{V}\right\rangle \sim 10.5 \mathrm{mag}$ and typical plasma energies $\langle\mathrm{kT}\rangle \sim 3 \mathrm{keV}$. The range of variability includes rapid impulsive flares and persistent low-level fluctuations indicative of strong magnetic activity, as well as slow rises and falls in count rate whose origin is more obscure. Some slowly-evolving outbursts reached sustained temperatures of $\mathrm{kT} \sim 6-10 \mathrm{keV}$. Chandra detected all but one of a subsample of 27 cTTS identified from previous near and mid-IR photometry, and their X-ray and bolometric luminosities are correlated. We also report the X-ray detection of IRS $2 \mathrm{~b}$, which is thought to be a massive embedded late O or early B star that may be the ionizing source of NGC 2024. Seven millimeter-bright cores (FIR 1-7) in NGC 2024 that may be protostellar were not detected, with the possible exception of faint emission near the unusual core FIR-4.
\end{abstract}


Subject headings: open clusters and associations: individual (NGC 2024) — stars: formation — stars: pre-main-sequence - X-rays: stars

\section{Introduction}

The conspicuous HII region NGC 2024 lies about 15' east of the bright O9.5Ib supergiant $\zeta$ Orionis in the Orion B molecular cloud (= Lynds 1630). Its estimated distance is $415 \mathrm{pc}$ (Anthony-Twarog 1982). Near-infrared (IR) observations have uncovered a remarkably dense cluster of young stars in the molecular cloud associated with NGC 2024 (Grasdalen 1974; Barnes et al. 1989 (B89); Comerón, Rieke, \& Rieke 1996; Lada et al. 1991; Haisch, Lada \& Lada 2000 (HLL); Haisch et al. 2001 (HLP); Meyer 1996 (M96)). The K-band survey of Lada et al. showed that the cluster covers an area of $\approx 180 \operatorname{arc}^{-m_{n}{ }^{2}}$ and contains more than 300 IR sources. The more sensitive survey of Comerón et al. detected $\approx 150$ sources in the

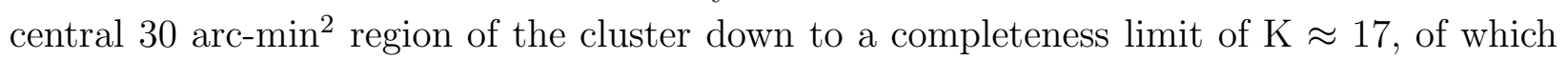
about two-thirds showed IR excesses characteristic of circumstellar disks. Using sensitive Lband observations, HLL found a somewhat higher infrared excess fraction $\geq 86 \%$. A recent near-IR adaptive optics survey detected 73 stars, of which 3 are binaries and one is a triple system (Beck, Simon, \& Close 2003 (B03)). Despite the high concentration of IR sources, there are no optically visible stars toward the cluster center. The cluster is undoubtedly young with age estimates ranging from $~ 0.3$ My (Meyer 1996) up to a few My (Comerón et al. 1996).

Millimeter and submillimeter observations provide evidence that star-formation is still underway in NGC 2024. Seven compact condensations of dust and gas denoted as FIR 1-7 were detected at $350-1300 \mu \mathrm{m}$ by Mezger et al. (1988 (M88), 1992 (M92)), who argued that the condensations are isothermal protostars. These compact cores have been subsequently studied by numerous authors as summarized by Lai et al. (2002). Their evolutionary status is still a subject of debate, but the discovery of a faint near-infrared source at the FIR 4 position (Moore \& Chandler 1989; Moore \& Yamashita 1995) and a unipolar redshifted outflow (Chandler \& Carlstron 1996) suggests active star formation in the vicinity of FIR 4. Polarization observations and Zeeman observations of absorption lines toward the cloud with radio interferometers have detected a magnetic field and there is some evidence that the field morphology around FIR 5 has been influenced by gravitational collapse (Crutcher et al. 1999; Lai et al. 2002).

At X-ray wavelengths, ROSAT observations of NGC 2024 were analyzed by Freyberg \& Schmitt (1995). The most sensitive image obtained was a $42 \mathrm{ks}$ HRI exposure in which 52 X-ray sources were detected. A shorter $22 \mathrm{ks}$ PSPC image showed that most X-ray sources 
have large absorption $\mathrm{N}_{\mathrm{H}} \sim 10^{22} \mathrm{~cm}^{-2}$ and typical unabsorbed X-ray luminosities were $\mathrm{L}_{\mathrm{x}}$ $\sim 10^{30} \mathrm{ergs} \mathrm{s}^{-1}$. The ROSAT detections were well-correlated with known K-band sources and the X-ray properties suggested that most were embedded young stars. The ROSAT observations clearly revealed a large population of X-ray emitting sources in NGC 2024. But, because of the heavy X-ray absorption below $\sim 1 \mathrm{keV}$ and rather limited PSPC spectral resolution and bandpass $(\approx 0.2-2.5 \mathrm{keV})$, spectral parameters for individual sources were not easily determined from $R O S A T$ data.

The NGC 2024 region is an ideal target for X-ray studies of star-formation for several reasons. It contains a diverse range of objects including class I protostars ${ }^{1}$, classical $\mathrm{T}$ Tauri stars (cTTS), weak-lined T Tauri stars (wTTS), the luminous infrared source IRS 2b which may be a massive young $\mathrm{OB}$ star, and seven millimeter condensations that may be protostellar. NGC 2024 lies at galactic coordinates $(b, l) \sim\left(-16^{\circ}, 207^{\circ}\right)$ so source confusion from the galactic plane or galactic center is not an issue. In addition, the high absorption toward the cluster center reduces background contamination.

We present here the results of a sensitive $76 \mathrm{ks}$ Chandra observation of NGC 2024 which provides higher angular and spectral resolution, broader energy coverage, and longer time monitoring than has previously been obtained. Our primary goals were: (i) to obtain a sensitive high angular resolution X-ray census of NGC 2024, (ii) to compare the X-ray population with existing IR catalogs and identify potential cloud members that might have escaped IR detection, (iii) to quantify the X-ray spectral and timing properties of the X-ray population, (iv) to determine if the total X-ray luminosity contributes significantly to the ionization of the visible HII region, and (v) to search for X-ray emission from the compact millimeter cores FIR 1-7. In the following we discuss the spatial, temporal, and spectral properties of the X-ray sources in NGC 2024 and compare the X-ray and infrared populations.

\section{Chandra Observation and Data Reduction}

\subsection{Observation}

Chandra observed NGC 2024 from 8 August 2001 at 06:37 UT until 04:29 UT on 9 August (ObsId 1878), spanning 21.9 hours. Exposures were obtained in faint data mode with a $3.2 \mathrm{~s}$ frame time using the ACIS-I imaging array as the primary detector. ACIS-I

\footnotetext{
${ }^{1}$ We adopt the the IR classification scheme used in Haisch et al. (2001) based on the IR spectral index $\alpha$ $=d \log \left(\lambda \mathrm{F}_{\lambda}\right) / d \log \lambda$ evaluated in the $2.2-10 \mu \mathrm{m}$ range. Class I sources have $\alpha>0.3$, flat spectrum sources have $-0.3 \leq \alpha<0.3$, class II sources have $-1.6 \leq \alpha<-0.3$, and class III sources have $\alpha<-1.6$.
} 
consists of four front-illuminated $1024 \times 1024$ pixel CCDs with a pixel size of $\sim 0.492^{\prime \prime}$ and a combined field-of-view $(\mathrm{FoV})$ of $\approx 16.9^{\prime} \times 16.9^{\prime}$. The S2 and S3 CCDs in the ACIS-S array were also enabled, but our discussion here will focus on the ACIS-I data. The exposure livetime varied slightly between CCDs but was in the range 76,655 - 76,658 s. The Chandra aimpoint was at nominal $\mathrm{RA}=5^{h} 41^{m} 46.3^{s}, \mathrm{DEC}=-1^{\circ} 55^{\prime} 11.7^{\prime \prime}$ (J2000), which is $\approx 32^{\prime \prime}$ from the strong millimeter source FIR-5 (M88). More detailed information on Chandra and its instrumentation can be found in the Chandra Proposer's Observatory Guide (POG) ${ }^{2}$.

\subsection{CIAO Data Reduction}

We followed standard data reduction procedures for ACIS data using CIAO software vers. 2.2.13 . Our data reduction was based on the Level 1 files generated during standard processing by the Chandra X-ray Center (CXC) using vers. 2.6 of the Chandra calibration database. The CIAO data reduction included (i) application of the most recent aspect and focal length corrections, (ii) event selection based on good $A S C A$ grades 0,2,3,4,6 and a clean status bit (status $=0$ ) and removal of afterglow events, and (iii) energy filtering. For image analysis and source identification we used events in the $0.5-7 \mathrm{keV}$ range to reduce particle background. Pixel randomization, which is introduced by $\mathrm{CXC}$ as part of the standard data processing to remove possible spatial aliasing effects, was retained. The algorithm to partially correct for the effects of charge transfer inefficiency (CTI) was not yet implemented in CIAO vers. 2.2.1 and we thus did not apply CTI corrections.

\subsection{Spatial Resolution and Limiting Sensitivity}

The on-axis spatial resolution of ACIS-I is limited by the physical pixel size of $\sim 0.492^{\prime \prime}$. For on-axis point sources, approximately $90 \%$ of the encircled energy lies within 4 pixels $\left(1.97^{\prime \prime}\right)$ of the center pixel at $1.49 \mathrm{keV}(P O G)$. The limiting sensitivity of ACIS depends on many factors including the exposure time, background level, location of the source on the CCD-array, and intrinsic source properties. To obtain a rough sensitivity estimate for this observation, we consider an on-axis point source and adopt a 6 count detection threshold (or $7.83 \times 10^{-2} \mathrm{c} / \mathrm{ks}$ for a livetime of $76.656 \mathrm{ks}$ ). We assume that the source has spectral properties typical of those sources detected in NGC 2024 (Sec. 4.3), namely an optically

\footnotetext{
${ }^{2}$ http://cxc.harvard.edu/udocs/docs/docs.html

${ }^{3}$ Further information on Chandra Interactive Analysis of Observations (CIAO) software can be found at http://asc.harvard.edu/ciao/
} 
thin thermal plasma with $\mathrm{kT} \approx 2.8 \mathrm{keV}$, absorption column density $\mathrm{N}_{\mathrm{H}}=2.3 \times 10^{22} \mathrm{~cm}^{-2}$, and abundances $\sim 0.3$ solar. For this typical source at an assumed distance of $415 \mathrm{pc}$, the unabsorbed luminosity detection limit from the Portable Interactive Multi-Mission Simulator $(P I M M S)$ software is $\log \mathrm{L}_{\mathrm{x}} \approx 28.8 \mathrm{ergs} \mathrm{s}^{-1}(0.5-7 \mathrm{keV})$. The detection limit is higher for off-axis sources, or for sources with heavier absorption or lower temperatures. Conversely, the detection limit is lower for sources having less absorption or higher temperatures.

\section{Data Analysis}

The X-ray data analysis consisted of (i) Chandra image generation and registration against IR positions from the 2MASS data base, (ii) identification of X-ray sources and their probable IR counterparts using existing catalogs, (iii) extraction and analysis of event lists for each source to determine source-specific quantities such as counts, mean photon energy, absorbed flux, and the Kolmogorov-Smirnov (KS) variability statistic, (iv) light curve extraction for all sources, and (v) extraction and fitting of spectra for brighter sources to obtain the absorption column density, characteristic plasma temperature, and unabsorbed luminosity. The analysis procedure is described in more detail below.

\subsection{Image Analysis and Source Identification}

We used the level 2 event file created by the CIAO processing described above to generate full-resolution $2800 \times 2800$ pixel images $\left(0.492^{\prime \prime}\right.$ pixels $)$ as well as binned images. Light curves from on-chip background regions were inspected for large background fluctuations that might have resulted from solar flares and none were found. To insure accurate identification of infrared counterparts, we registered Chandra positions against IR positions in the the 2MASS database ${ }^{4}$. To do this, we identified approximately 100 2MASS sources with obvious counterparts in the initial Chandra images. We then used the ASTROM program in the Starlink software package to derive a 4-coefficient plate scale solution using 2MASS J2000 positions and corresponding Chandra physical pixel positions. After applying the positional correction to the Chandra data, we found typical offsets between X-ray and 2MASS positions

near the center of the ACIS-I detector to be $\sqrt{\Delta \mathrm{RA}^{2}+\Delta \mathrm{DEC}^{2}} \approx 0.25^{\prime \prime}$. These small offsets permitted IR counterparts to be identified with very high confidence.

After applying the astrometric correction, we used the CIAO wavelet source detection

\footnotetext{
${ }^{4}$ http://www.ipac.caltech.edu/2mass/
} 
program WAVDETECT to search for X-ray sources. Several WAVDETECT passes were made using scale factors of 2,4 , and 8 and false-alarm probabilities of $10^{-5}$ and $10^{-6}$. The source lists from each WAVDETECT pass were compared and the Chandra images were visually inspected for missed or spurious detections.

We identified 283 X-ray sources of which 248 were associated with known IR, optical, or radio counterparts based on searches of the SIMBAD data base, the 2MASS all-sky release Point Source Catalog, source lists from previous IR studies (HLL; HLPTL; B89; B03; M96), and a list of radio detections obtained in a recent $3.6 \mathrm{~cm}$ VLA survey conducted by Rodriguez et al. (2003). Table 1 lists the X-ray detections and their counterparts along with other source information. Table 1 is also supplied in electronic format.

The positions and PSF-corrected 95\% encircled energy regions around each source were used to extract source events. For the brightest sources a significant number of photons can fall outside the $95 \%$ encircled energy region, so larger $99 \%$ encircled energy regions were used for event extraction in bright sources. For on-axis sources the $95 \%$ and $99 \%$ encircled energy regions are nearly circular and have radii $\mathrm{R}_{95 \mathrm{EE}} \approx 2^{\prime \prime}$ and $\mathrm{R}_{99 \mathrm{EE}} \approx 8^{\prime \prime}$ (Feigelson et al. 2002). In the crowded cluster core region, it was necessary in some cases to use smaller extraction regions to avoid overlap from nearby sources.

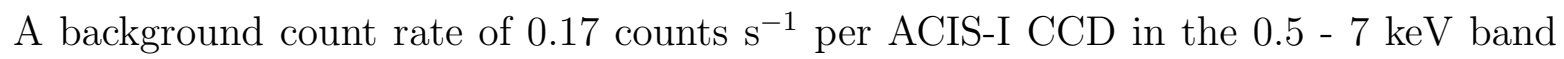
(Table 6.6 of $P O G$ ) was used along with the size of the source extraction region and exposure time to estimate the number of background counts for each source. The net source counts were then obtained by subtracting the number of background counts from the total number of counts in the source extraction region. The net counts for each source are given in Table 1 along with other source information. The number of source counts that might have fallen outside the extraction region is within the net count uncertainty quoted in Table 1. Sources with fewer than 6 net counts were not included in Table 1.

Figure 1 shows a smoothed broad-band Chandra image of a $4^{\prime} \times 4^{\prime}$ region in the central part of the cluster where the X-ray source density is highest. Figure 2 is an unsmoothed image of the region in the vicinity of the infrared source IRS $2 \mathrm{~b}$, the possible ionizing source of NGC 2024 (Bik et al. 2003). Figure 3 is a zoomed smoothed broad-band image of the region near the millimeter sources FIR 1-7, which were not detected by Chandra with the possible exception of weak emission near FIR-4. This weak emission (4 counts) is shown in Figure 4. The astrometric-corrected positions of all 283 Chandra detections are plotted in Figure 5, overlaid with the positions of all 912 catalogued IR sources in the ACIS-I FoV. The X-ray source density is clearly higher near the center of the FoV, within the boundaries of the known IR cluster. 


\subsubsection{Unidentified Chandra Sources}

Figure 6 shows the positions of the 35 X-ray sources which lack counterparts at other wavelengths. About one-third of the unidentified sources have positions projected within the boundaries of the IR cluster and could be cluster members. However, the majority of the unidentified sources are probably extragalactic.

The contribution of X-ray emitting foreground stars to the unidentified sample is expected to be small because of the absence of optical counterparts (see also Barger et al. 2002 for a breakdown of Chandra Deep Field North sources by object type). The faint hard emission of the unidentified X-ray sources also suggests a predominantly extragalactic origin. None of the 35 unidentified sources has more than 100 counts, while $43 \%$ of the identified sources have more than 100 counts. All but four of the unidentified sources have mean photon energies above the average computed for all X-ray detections (Sec. 4.2).

Using the 2 - $8 \mathrm{keV}$ X-ray number counts from Chandra Deep Field observations (Cowie et al. 2002), we estimate $\sim 16$ extragalactic sources in the ACIS-I FoV above our detection limit. This estimate should be treated with caution since (i) a reduction in the number of background sources detected toward NGC 2024 will occur as a result of absorption below a few keV from intervening molecular cloud material, which is non-uniform due to the northsouth molecular ridge that transects NGC 2024, and (ii) the accuracy of the $\log \mathrm{N}-\log \mathrm{S}$ distribution for extragalactic background sources from Chandra Deep Field observations at the lower Galactic latitude observed here $\left(b \approx-16^{\circ}\right)$ is not yet known, as already pointed out in a discussion of Chandra Orion Nebula observations (Feigelson et al. 2002).

In Figure 6, we have circled six unidentified Chandra sources that could be extragalactic based on their exceptionally faint ( $\leq 25$ counts), hard $(\langle\mathrm{E}\rangle>4 \mathrm{keV})$, non-variable emission. Excluding these extragalactic candidates, there are seven remaining unidentified sources within the approximate IR cluster boundaries that may be young stars and for which followup observations at other wavelengths would be useful. These are sources 20, 21, 157, 168, 180, 197, and 223. The latter source may have a 2MASS IR counterpart (Table 1).

\subsection{Timing Analysis}

To obtain a quantitative measurement of the variability for each source, we have computed the non-parameteric Kolmogorov-Smirnov (KS) statistic from the event list of each source (Table 1 ). The KS statistic is given by $\mathrm{KS}=\sqrt{n} \sup \left|f_{i}(t)-f_{0}(t)\right|$ where $n$ is the number of events, $f_{i}(t)$ is the normalized observed cumulative distribution and $f_{0}(t)$ is the normalized model cumulative distribution, assuming a constant flux. A discussion of the KS 
method and a comparison with the more familiar $\chi^{2}$ test is given by Rohatgi (1976) and additional information can be found in Babu \& Feigelson (1996) and Press et al. (1992).

If $z$ is the value of the KS statistic then the null hypothesis probability $\mathrm{P}(z)$, or probability of constant flux, is given by a convergent infinite series (Press et al. 1992):

$$
\mathrm{P}(z)=2 \sum_{j=1}^{\infty}(-1)^{j-1} e^{-2 j^{2} z^{2}}
$$

Truncating the series at six terms gives a few representative values: $\mathrm{P}(0.5)=0.96$, $\mathrm{P}(1.0)=0.27, \mathrm{P}(1.5)=0.02, \mathrm{P}(1.7)=0.006$, and $\mathrm{P}(2.0)=6.7 \times 10^{-4}$. In the discussion below we adopt $z=\mathrm{KS}=1.7$ as the dividing line between variable and non-variable sources, where $\mathrm{KS}=1.7$ implies a variability probability $\mathrm{P}_{v a r}=0.994$. That is, any source in Table 1 with KS > 1.7 is considered to be variable. The KS values in Table 1 can be converted to the corresponding null hypothesis probability using the above equation. We note that the KS statistic is less reliable for diagnosing variability in weak sources $(<25$ counts) as discussed further below (Sec. 4.1).

The KS statistic identifies those sources that were variable but does not provide information on variability profiles or timescales. To obtain this information, light curves were generated for all sources. Figure 7 shows light curves for ten sources in the ACIS-I FoV that demonstrate the wide range of variability detected. Figure 8 illustrates the continuous variability that was detected in source 94 throughout the observation. In Figure 9, we show the hard variable emission in source 207 with mean photon energies in excess of $4 \mathrm{keV}$ and very high plasma temperatures. The interesting variability in these sources is discussed in more detail below (Sec. 4.5).

\subsection{Spectral Analysis}

We have used two different approaches to determine source spectral properties. First, the event list of each source was analyzed to determine the mean photon energy $\langle\mathrm{E}\rangle$ and the

absorbed flux $\mathrm{F}_{\mathrm{x} \text {,abs }}(0.5-7 \mathrm{keV})$ values listed in Table 1 . Second, spectra were extracted for the brightest sources and fitted with thermal plasma models to determine the absorption column density $\left(\mathrm{N}_{\mathrm{H}}\right)$, plasma temperature $(\mathrm{kT})$, and unabsorbed luminosity $\mathrm{L}_{\mathrm{x}}(0.5-7 \mathrm{keV})$ values given in Table 1.

These two methods are complementary. The advantage of event list analysis is that it 
provides information on spectral hardness (or $\langle\mathrm{E}\rangle$ ) and $\mathrm{F}_{\mathrm{x}, \text { abs }}$ for all detections, including faint sources. Spectral fitting provides additional information on $\mathrm{N}_{\mathrm{H}}, \mathrm{kT}$, and $\mathrm{L}_{\mathrm{x}}$ but is only practical for brighter sources ( $\geq 90$ counts).

The advantage of using both techniques is that the column density $\mathrm{N}_{\mathrm{H}}$ from spectral fits of brighter sources can be incorporated into the event list analysis. In that case, the event list analysis provides information on $\mathrm{kT}$ and $\mathrm{L}_{\mathrm{x}}$, as well as $\langle\mathrm{E}\rangle$ and $\mathrm{F}_{\mathrm{x} \text {,abs. }}$. If the event list of a specific source is first partitioned by time, then the time evolution of these four quantities can be plotted in "light curve" format, as shown in Figures 8 and 9.

Event List Analysis: Our event list analysis followed the procedures described in Gagné, Daniel, \& Skinner (2003), which we summarize here. The mean energy $\langle\mathrm{E}\rangle$ is obtained from the event list, which contains the arrival time and energy of each photon. To compute the unabsorbed flux $F_{x, a b s}$, we generated an auxiliary response file (ARF) for each source, which contains information on effective area as a function of photon energy, taking into account the source position on the detector. The absorbed flux is then given by

$$
F_{\mathrm{x}, \mathrm{abs}}=\frac{1}{t} \sum_{i=1}^{n} \frac{E_{i}}{a_{i}(E)}-F_{\mathrm{bkg}} A,
$$

where $E_{i}$ is the energy of each photon, $a_{i}(E)$ is the corresponding effective area, $t$ is the livetime of the observation, $\mathrm{A}$ is the area of the source extraction region, and $F_{\mathrm{bkg}}=3.84 \times$ $10^{-18} \mathrm{ergs}^{-2} \mathrm{~s}^{-1}$ pixel $^{-1}$ is the $0.5-7.0 \mathrm{keV}$ background flux as determined from the CXC software tool MAKE_ACISBG.

For brighter sources with $\geq 90$ counts, additional information on the column density $\mathrm{N}_{\mathrm{H}}$ is available from spectral fits (see below) and the event list analysis can be taken a step further to obtain plasma temperature $\mathrm{T}$ and $\mathrm{L}_{\mathrm{x}}$. This is accomplished by generating a grid of simulated spectra using the $1 \mathrm{~T}$ VAPEC model in XSPEC vers. 11, with column densities in the range $\log \mathrm{N}_{\mathrm{H}}=20.0-24.0 \mathrm{~cm}^{-2}$ and temperatures in the range $\log \mathrm{T}=6.0-9.0$ $(\mathrm{K})$ in increments of $0.1 \mathrm{dex}$. The grid is parameterized as a function of the mean photon energy $\langle\mathrm{E}\rangle$, which is known from the event list of each source. Simulations show that $\langle\mathrm{E}\rangle$ increases smoothly with $\mathrm{N}_{\mathrm{H}}$ and $\mathrm{T}$. The grid is then entered with the values of $\mathrm{N}_{\mathrm{H}}$ and $\langle\mathrm{E}\rangle$ to determine $\mathrm{T}$. Once $\mathrm{T}$ is determined it can be used along with $\mathrm{N}_{\mathrm{H}}$ to compute the conversion factor from absorbed to unabsorbed flux $\mathrm{C}\left(\mathrm{T}, \mathrm{N}_{\mathrm{H}}\right)=\mathrm{F}_{\mathrm{x}} / \mathrm{F}_{\mathrm{x} \text {,abs }}$. The unabsorbed luminosity is then $\mathrm{L}_{\mathrm{x}}=4 \pi \mathrm{d}^{2} \mathrm{~F}_{\mathrm{x}}=4 \pi \mathrm{d}^{2} \mathrm{~F}_{\mathrm{x}, \text { abs }} \mathrm{C}\left(\mathrm{T}, \mathrm{N}_{\mathrm{H}}\right)$.

Spectral Fits: Spectra for sources with $\geq 90$ counts were extracted along with source-specific response matrix files (RMF) and ARF files using the CIAO tool PSEXTRACT. Background spectra were extracted from regions in the immediate vicinity of the source. Spectra of 
weaker sources with $\sim 90$ - 300 counts were rebinned to 10 counts per bin prior to spectral fitting, while brighter sources (>300 counts) were rebinned to 15 counts per bin. We have restricted our spectral analysis to sources with $\geq 90$ counts since our test fits show that the uncertainties in spectral parameters derived for weaker sources are often too large to permit definitive analysis.

All sources with $\geq 90$ counts were fitted with a single-temperature absorbed optically thin plasma model (1T VAPEC) using XSPEC vers. 11. A representative $1 \mathrm{~T}$ VAPEC fit of variable source 85 is shown in Figure 10, along with its pre-outburst spectrum. Brighter sources (>300 counts) were also fitted with a two-temperature 2T vAPEC model and in some cases the differential emission measure (DEM) model C6PVMKL was applied. During spectral fitting with VAPEC models, the free parameters were the equivalent neutral hydrogen column density $\left(\mathrm{N}_{\mathrm{H}}\right)$, X-ray temperature(s) (kT), and normalization factors used to reproduce the observed count rate.

Abundances were held fixed at solar values relative to Anders \& Grevesse (1989), except for iron which was fixed at a lower value $\mathrm{Fe}=0.3 \times$ solar. The choice of a subsolar iron abundance was based on variable abundance fits of the brightest sources which invariably converged to a low Fe abundance near $\mathrm{Fe} \approx 0.3 \times$ solar. For example, we cite the relatively strong non-variable source 75 (3092 counts). Fits of this source with a $1 T$ VAPEC model converged to $\mathrm{Fe}=0.22[0.04-0.40]$ solar and $2 \mathrm{~T}$ VAPEC models gave a very slight improvement with $\mathrm{Fe}=0.35[0.13-0.58]$ solar, where brackets enclose $90 \%$ confidence intervals. A comparison of $1 \mathrm{~T}$ VAPEC fits of different sources indicates that solar abundance models tend to overestimate $\mathrm{N}_{\mathrm{H}}$ and unabsorbed $\mathrm{L}_{\mathrm{x}}$ relative to the improved fits obtained with subsolar Fe.

The unabsorbed X-ray flux in the $0.5-7 \mathrm{keV}$ range was measured from best-fit models after setting $\mathrm{N}_{\mathrm{H}}=0$ and then converted to an equivalent unabsorbed luminosity $\mathrm{L}_{\mathrm{x}}$ using a distance of 415 pc. Best-fit values of $\mathrm{N}_{\mathrm{H}}$ and $\mathrm{kT}$ (with $90 \%$ confidence limits from the XSPEC steppar routine) and unabsorbed $\mathrm{L}_{\mathrm{x}}$ for the brightest sources are given in Table 1 .

We emphasize that $\mathrm{kT}$ values determined from the single-temperature $1 \mathrm{~T}$ VAPEC models are only used to define a characteristic time-averaged temperature that allows a large number of sources to be compared. This should not be construed to mean that the X-ray emitting plasma is isothermal. It is clear from our analysis that large temperature fluctuations did occur in some sources (Table 1 notes; see also Figs. 8 and 9). However, in sources with no statistically significant variability we only found definite improvement in spectral fits using a two-temperature model (2T VAPEC) in a few cases (Table 1 notes). This may be attributed to the strong absorption below $\sim 1 \mathrm{keV}$ that is present in most spectra, effectively masking any soft emission that could be present. The spectrum of source 85 in Figure 10 clearly 
illustrates this low-energy absorption.

\subsection{Implications of Photon Pileup}

Pileup occurs when two or more photons are detected within the same pixel during one 3.2 s readout period. Pileup is a consideration for high count-rate sources at small off-axis angles where the point-spread function (PSF) is narrowest, even for rapidly flaring sources where the count rate may increase to high levels only for short periods of time. In general, the effects of pileup are to make the spectrum appear harder than it actually is and to artificially reduce the count rate. However, there are other implications as discussed in the Chandra POG.

We have identified those bright sources in NGC 2024 where significant pileup (>10\%) could have occurred. Fortunately, pileup is not an issue for this observation except for a few sources, and then only at moderate levels. Three strongly variable sources located within $\approx 1^{\prime}$ of the Chandra aimpoint had peak count rates that exceeded $\approx 0.09 \mathrm{c} \mathrm{s}^{-1}$ and are suspected to have piled at the $\approx 10 \%-30 \%$ level for short periods. These sources are nos. 174, 192, and 210. Another source which had a relatively high but steady count rate was no. 120, whose mean count rate was $0.10 \mathrm{c} \mathrm{s}^{-1}$ with short-term fluctuations up to $\approx 0.15 \mathrm{c} \mathrm{s}^{-1}$. However, it is offset $\approx 2^{\prime}$ from the aimpoint so its pileup should not have exceeded $\approx 10 \%-15 \%$ due to off-axis broadening of the PSF. Because of moderate pileup in these four sources, their mean photon energies and flare temperatures given in Table 1 may be slightly overestimated, and total counts may be slightly underestimated.

\section{X-ray Properties}

We summarize here the global X-ray properties of the Chandra sources in NGC 2024 based on temporal and spectral analysis.

\subsection{Time Variability}

A total of 72 out of 283 sources (25\%) showed variability as inferred from $\mathrm{KS}>1.7$, and further information on sources with the strongest variability is given in the notes to Table 1. However, the variability fraction appears to be much larger among brighter sources. Only

$5 \%$ of those sources with less than 25 counts showed variability, whereas $64 \%$ of sources with more than 400 counts were variable. This trend is obvious in Figure 11, which shows a 
monotonic increase in variability fraction with source counts. On physical grounds, there is little reason to suspect that the variability fraction should increase monotonically with the number of source counts. Instead, this trend can be attributed to the increased difficulty in detecting variability in weaker sources. It is thus likely that the true variability fraction of the 283 detections is greater than $25 \%$ due to undetected variability in faint sources, and this value should be interpreted as a lower limit.

The range of variability shown in the light curves (Fig. 7) is truly amazing, consisting of rapid onset flares (e.g. sources 27, 81 and 174), variations with slow rises and decays (175, 192, and 207), and sources with slowly declining or increasing count rates throughout the observation $(94,123,269)$. Although rapid-onset flares are suggestive of solar-like behavior attributable to magnetic reconnection events, the origin of the slower variability is more obscure. In some cases the slow variability may be dynamically-induced, as for example by rotation of an X-ray bright active region on the star across the line-of-sight. Longer observations spanning days or weeks would be needed to search for periodic variability.

\subsection{Spectral Hardness (Mean Photon Energy)}

Figure 12 shows the distribution of mean photon energy $\langle\mathrm{E}\rangle$ for all 283 detections. The average value is $\langle\langle\mathrm{E}\rangle\rangle=2.7 \mathrm{keV}$. The distribution in Figure 12 is noticeably truncated at low energies below $\langle\mathrm{E}\rangle \sim 1.4 \mathrm{keV}$. This is a result of the heavy absorption toward the cloud which effectively absorbs soft photons and skews the distribution.

Figure 13 is a plot of $\langle\mathrm{E}\rangle$ against the $\mathrm{KS}$ variability statistic. We have partitioned the diagram into four quadrants using $\mathrm{KS}=1.7$ to separate variable and non-variable sources and $\langle\mathrm{E}\rangle=2.7 \mathrm{keV}$ to distinguish between sources with above average or below average mean photon energies.

Several interesting features are apparent in Figure 13. First, all four quadrants are well-populated and there is no obvious relationship between variability $(\mathrm{KS})$ and $\langle\mathrm{E}\rangle$ for the sample as a whole. It is apparent that variability is not confined to hard sources since many sources with KS $<1.7$ have $\langle\mathrm{E}\rangle>2.7 \mathrm{keV}$. The Kendall's $\tau$ test in the ASURV statistical software package gives a probability of correlation between KS and $\langle\mathrm{E}\rangle$ of $p=0.74$ and the Cox test gives $p=0.93$. Thus, statistical analysis does not provide conclusive evidence for a correlation. Second, it is obvious from Figure 13 that most of the 35 unidentified Chandra sources lie in the lower right part of the diagram and have hard non-variable emission. These sources are also faint (Sec. 3.1.1) and the hard emission suggests that most are extragalactic background sources but some could be deeply embedded sources in the cloud. Third, there 
is a large spread in $\langle\mathrm{E}\rangle$ among the Chandra sources, ranging from $\langle\mathrm{E}\rangle=0.92 \mathrm{keV}$ (source 181, which has the IR counterpart HLL-116) to the faint hard non-variable source 197 with $\langle\mathrm{E}\rangle=4.77 \mathrm{keV}$ and no known counterpart.

\subsection{Spectral Temperature and Absorption Estimates}

Since very few of the Chandra detections have optical counterparts, reliable information on the visual extinction $A_{V}$ is generally not available. We have not made extensive use of methods for estimating $A_{V}$ based on JHK photometry since these methods make a priori assumptions about dereddened colors of the star in order to extract the color excess and $A_{V}$. The errors which can be incurred in $A_{V}$ are substantial, as discussed in more detail by Gagné et al. (2003).

Figure 14 is a plot of $\mathrm{N}_{\mathrm{H}}$ and $\mathrm{kT}$ derived from spectral fits of more than 100 of the brightest X-ray sources. It is apparent from Figure 14 that those sources with the highest absorption and highest temperatures are all variable. However, the converse is not true. Variability was also detected in sources that are situated in the lower left part of the diagram, where $\mathrm{kT}$ and $\mathrm{N}_{\mathrm{H}}$ are relatively low. Thus, X-ray variability alone does not provide reliable information on either time-averaged temperature or absorption.

The median absorption determined from 117 sources is $\log \mathrm{N}_{\mathrm{H}}=22.18 \mathrm{~cm}^{-2}$ and the mean is $\log \left\langle\mathrm{N}_{\mathrm{H}}\right\rangle=22.37 \mathrm{~cm}^{-2}$. The average temperature derived from $1 \mathrm{~T}$ VAPEC fits of sources with $\geq 90$ counts is $\langle\mathrm{kT}\rangle=2.8 \mathrm{keV}$. In computing this average we have attempted to exclude time intervals during large rapid flares or slowly varying outbursts, although this was not possible in some cases where non-flare counts were low.

The mean absorption column density $\log \left\langle\mathrm{N}_{\mathrm{H}}\right\rangle=22.37 \mathrm{~cm}^{-2}$ corresponds to a mean visual extinction $\left\langle\mathrm{A}_{V}\right\rangle=10.5 \mathrm{mag}$ using the conversion $\mathrm{A}_{V}=4.5 \times 10^{-22} \mathrm{~N}_{\mathrm{H}}$ mag (Gorenstein 1975). This $\mathrm{X}$-ray derived value is in excellent agreement with the value $\mathrm{A}_{V} \approx 10.5$ mag determined for cluster members using IR data (HLL). We note a rather large spread in $\mathrm{N}_{\mathrm{H}}$ ranging from no detectable absorption in source 282 (= 2MASS 05422123-0159104) to a maximum of $\log \mathrm{N}_{\mathrm{H}}=23.18 \mathrm{~cm}^{-2}\left(\mathrm{~A}_{V} \approx 68 \mathrm{mag}\right)$ in the variable source 160 .

Estimates of $\mathrm{A}_{V}$ based on IR data have been derived for 27 class II sources (cTTS) in NGC 2024 and the class III source (wTTS) HLP-24, as listed in Table 2 of HLP. We derived independent estimates of $\mathrm{N}_{\mathrm{H}}$ for 21 of the class II sources from Chandra spectral fits (Table 1). Using the results of Gorenstein (1975) to convert from $\mathrm{N}_{\mathrm{H}}$ to $\mathrm{A}_{V}$, we generally find good agreement between the X-ray and IR determinations of $\mathrm{A}_{V}$ subject to rather large uncertainties in the X-ray estimates for fainter sources. But significant differences were found 
for two sources. For source 31 (187 counts) whose counterpart is the IR source HLP-64, we obtain $\mathrm{A}_{V}=3.1\left[1.8-4.5\right.$; $90 \%$ conf.] mag while the IR data give $\mathrm{A}_{V}=7.1 \mathrm{mag}$. For source 69 (115 counts) the counterpart is HLP-14 which lies in a crowded IR field and the X-ray data yield $\mathrm{A}_{V}=5.8[3.6-8.6]$ mag but the IR data give a smaller value $\mathrm{A}_{V}=0.8$ mag.

\subsection{X-ray Luminosities}

Since reliable estimates of the visual extinction are not available for most of the embedded sources in NGC 2024, the intrinsic (unabsorbed) X-ray luminosities of many of the fainter Chandra detections are not yet known. We thus restrict our discussion of X-ray luminosities to the subsample of class II and flat-spectrum sources for which $\mathrm{A}_{V}$ estimates are available, and to an estimate of the total cluster X-ray luminosity.

\subsubsection{X-ray Luminosities of Class II Sources (cTTS)}

NGC 2024 contains 27 class II sources identified from previous near and mid-IR observations (Table 2 of HLP). Their spectral energy distributions peak in the near IR and decline more slowly toward the mid-IR than do normal stellar photospheres, indicative of circumstellar disks.

Chandra detected 26 of the 27 class II sources listed in HLP. The only class II source not clearly detected was HLP-100 (= 2MASS 05413545-0152288). However, we do see a weak X-ray source (5 counts) at an offset of $0.75^{\prime \prime}$ from HLP-100, and we classify it as a possible detection.

Figure 15 shows the cumulative X-ray luminosity distribution function for the 27 class II sources. This distribution function was obtained using the Kaplan-Meier (KM) estimator in the ASURV software package. We have estimated unabsorbed $L_{x}$ for the faintest class II detections using the PIMMS simulator and the absorbed fluxes in Table 1. The PIMMS simulations assumed a $1 \mathrm{~T}$ Raymond-Smith optically thin plasma model with a typical temperature for sources in NGC 2024 and an absorption $\mathrm{N}_{\mathrm{H}}$ computed from the $\mathrm{A}_{V}$ values given in HLP and the Gorenstein (1975) transformation. The possible detection of HLP-100 was treated as an upper limit (5 counts) with $\log \mathrm{L}_{\mathrm{x}}(0.5-7 \mathrm{keV}) \leq 28.63 \mathrm{ergs} \mathrm{s}^{-1}$.

A hard tail is clearly evident in the luminosity distribution function shown in Figure 15. This is due to the anomalously high $\mathrm{L}_{\mathrm{x}}$ values of four class II sources with $\mathrm{L}_{\mathrm{x}} \geq 30.7$ ergs $\mathrm{s}^{-1}$ which exhibited rapid-onset flares or other prominent variability (source nos. 41, 94, 133, 174). 
Estimates of the bolometric luminosity $\left(\mathrm{L}_{b o l}\right)$ were given for the 27 class II sources by HLP, ranging from $\mathrm{L}_{b o l}=61 \mathrm{~L}_{\odot}$ for HLP-74 (source 174 ) to $\mathrm{L}_{b o l}=1 \mathrm{~L}_{\odot}$ for both HLP98 (source 220) and HLP-100 (not detected). We used their $\mathrm{L}_{b o l}$ values along with the unabsorbed $\mathrm{L}_{\mathrm{x}}(0.5-7 \mathrm{keV})$ measurements from Chandra to check for a correlation and to obtain a linear regression fit using the Buckley-James algorithm in ASURV. We excluded the four strongly variable sources noted above from the correlation test. The Kendall's $\tau$ and Cox tests both give a correlation probability $p=0.98$ between $\mathrm{L}_{b o l}$ and unabsorbed $\mathrm{L}_{\mathrm{x}}(0.5$ $7 \mathrm{keV})$. The regression analysis gives $\log \mathrm{L}_{\mathrm{x}}\left(\operatorname{ergs~s}^{-1}\right)=29.86+0.54( \pm 0.19) \log \left[\mathrm{L}_{b o l} / \mathrm{L}_{\odot}\right]$, with a standard deviation of 0.36 in $\log \mathrm{L}_{\mathrm{x}}$. For a typical class II source with $\mathrm{L}_{b o l} \sim 1 \mathrm{~L}_{\odot}$ this relation yields $\log \left[\mathrm{L}_{\mathrm{x}} / \mathrm{L}_{b o l}\right] \sim-3.7$.

We consider the results of the above regression fit to be preliminary since it is based only on the IR-bright $(\mathrm{K} \leq 10.5)$ class II sources in NGC 2024 identified by HLP and lacks information on faint sources whose IR classification is not yet known. Even so, the regression fit is consistent at the $\approx 1 \sigma$ level with results from other young clusters having more complete optical/IR data such as IC 348 (Preibisch \& Zinnecker 2002), and is nearly identical to the average $\left\langle\log \left[\mathrm{L}_{\mathrm{x}} / \mathrm{L}_{b o l}\right]\right\rangle=-3.9 \pm 0.7$ found for a much larger sample of young stars in the Orion Nebula Cluster (Feigelson et al. 2002). Thus, based on this preliminary analysis, we find no reason to believe that the $\mathrm{L}_{\mathrm{x}} / \mathrm{L}_{b o l}$ ratios of class II sources in NGC 2024 are substantially different from those in other young clusters.

Previous studies of star-forming regions have reported correlations $\mathrm{L}_{\mathrm{x}} \propto \mathrm{L}_{\mathrm{J}} \propto \mathrm{L}_{b o l}$, where $\mathrm{L}_{\mathrm{J}}$ is the J-band luminosity (Casanova et al. 1995). Since the soft X-ray absorption in the energy range $\mathrm{kT} \approx 1.1-1.4 \mathrm{keV}$ is comparable to that at J-band, it is possible to compare the observed (absorbed) soft-band X-ray and J-band luminosities. To compare with results for other star-forming regions, we have plotted the absorbed luminosity $\mathrm{L}_{\mathrm{x}, \mathrm{abs}}(1.1$ - $1.4 \mathrm{keV}$ ) versus observed J magnitude from HLP for the known class II sources in NGC 2024 (Fig. 16). Neither parameter has been corrected for extinction. We have excluded the four highly-variable class II sources noted above. There is a clear trend for brighter J-band sources to have larger $\mathrm{L}_{\mathrm{x}, \text { abs }}$ with the notable exception of one outlier, namely the bright IR source HLP-2 (Chandra no. 77). Excluding the outlier and the four strongly variable sources, the Cox and Kendall's $\tau$ tests give a probability of correlation $p=0.99$ between observed $\mathrm{J}$ magnitude and $\mathrm{L}_{\mathrm{x}, \text { abs }}$. Using the Buckley-James algorithm and again excluding the outlier, a regression fit gives $\log \mathrm{L}_{\mathrm{x}, \text { abs }}[1.1-1.4 \mathrm{keV}]\left(\operatorname{ergs~s}^{-1}\right)=-0.37( \pm 0.11)(\mathrm{J}-12)$ +28.27 with a standard deviation of 0.56 in $\log \mathrm{L}_{x, a b s}$. Considering the uncertainties, the above regression slope $-0.37( \pm 0.11)$ agrees quite well with the results for other star-forming regions (Casanova et al. 1995; Getman et al. 2002).

It is noteworthy that several studies have shown obvious exceptions to the $\mathrm{L}_{\mathrm{x}, \mathrm{abs}} \propto \mathrm{L}_{\mathrm{J}}$ 
relation. In NGC 2024, the IR-bright source HLP-2 $(\mathrm{J}=7.93, \mathrm{~K}=6.56)$ is more than an order of magnitude less luminous in $\mathrm{L}_{\mathrm{x} \text {,abs }}$ than predicted by the above regression fit. We have undertaken an analysis similar to the above using $\mathrm{K}$ magnitudes and observed X-ray fluxes in the harder $3.2-4.2 \mathrm{keV}$ band, for which the absorptions are comparable. Again, HLP-2 shows up as an outlier with a luminosity $\mathrm{L}_{\mathrm{x}, \mathrm{abs}}(3.2-4.2 \mathrm{keV})$ that is at least an order of magnitude below that expected for its $\mathrm{L}_{\mathrm{K}}$. Exceptions to the $\mathrm{L}_{\mathrm{x}, \mathrm{abs}} \propto \mathrm{L}_{\mathrm{J}}$ relation were also seen in NGC 1333 (Fig. 11 of Getman et al. 2002) and the $\rho$ Ophiuchi core (Fig. 4 of Casanova et al. 1995). The exceptions that are bright at J-band but weak in X-rays such as HLP-2 and the objects SR1 and SR3 in $\rho$ Ophiuchi appear to have high bolometric luminosities and are thus more massive stars or perhaps unresolved multiple systems.

\subsubsection{X-ray Luminosities of Flat-Spectrum Sources}

Six flat-spectrum sources were identified in Table 1 of HLP, of which three were detected by Chandra. These were HLP-1 (source 187), HLP-12 (source 71), and HLP-38 (source 125). HLP-1 is the brightest K-band source in the HLP catalog $(\mathrm{K} \approx 5.5-5.8)$. The IR-bright flat-spectrum source HLP-6 was not detected but it fell in a gap between CCDs. Although the sample is small, the $50 \%$ detection rate of these six flat-spectrum sources is lower than the $96 \%$ detection rate for known class IIs. The X-ray luminosities of the three flat-spectrum detections were similar and in the range $\log \mathrm{L}_{\mathrm{x}}=30.14-30.37 \mathrm{ergs} \mathrm{s}^{-1}$ (Table 1). Because of the small sample size, statistical analysis of the flat-spectrum sources was not undertaken.

\subsubsection{Ionizing Source and Total Cluster X-ray Luminosity}

The mean absorbed X-ray flux of all 283 detections from Table 1 is $\left\langle\mathrm{F}_{\mathrm{x}, \mathrm{abs}}(0.5-7\right.$ $\mathrm{keV})\rangle=4.6 \times 10^{-14} \mathrm{ergs} \mathrm{cm}^{-2} \mathrm{~s}^{-1}$. Assuming a $1 \mathrm{~T}$ optically thin plasma model with source properties typical of NGC $2024\left(\mathrm{kT}=2.8 \mathrm{keV}, \mathrm{N}_{\mathrm{H}}=2.3 \times 10^{22} \mathrm{~cm}^{-2}\right)$, the corresponding mean unabsorbed flux is $\left\langle\mathrm{F}_{\mathrm{x}}(0.5-7 \mathrm{keV})\right\rangle=1.2 \times 10^{-13} \mathrm{ergs} \mathrm{cm}^{-2} \mathrm{~s}^{-1}$ which equates to an unabsorbed luminosity $\left\langle\mathrm{L}_{\mathrm{x}}(0.5-7 \mathrm{keV})\right\rangle=2.5 \times 10^{30} \mathrm{ergs} \mathrm{s}^{-1}$. This average is about 1300 times larger than the X-ray luminosity of the quiet Sun, underscoring the prodigious X-ray output of pre-main-sequence stars. Summing over 283 sources gives a total luminosity $\mathrm{L}_{\mathrm{x}, \mathrm{tot}}$ $(0.5-7 \mathrm{keV}) \sim 7 \times 10^{32} \mathrm{ergs} \mathrm{s}^{-1}$.

The source of the ionizing radiation which excites NGC 2024 has been difficult to identify but previous studies based on energy considerations have concluded that the exciting star is most likely of spectral type B0 - O9 (Hjellming 1968; Gordon 1969; Thronson et al. 1984). 
Although the O9.5Ib star $\zeta$ Ori may contribute to the ionization, it lies $\sim 15^{\prime}$ away and is not believed to be the primary excitation source (Gordon 1969; B89). The lack of an obvious ionizing source led Barnes et al. (1989) to suggest that the combined contribution from the embedded IR population, rather than a putative B0-O9 star, might be the source of the ionization. It is thus relevant to ask if the total X-ray emission associated with the cloud population might be an important contribution to the ionization. Our Chandra observation shows that the known X-ray population could contribute $\mathrm{L}_{\mathrm{x}, \mathrm{tot}} \sim 10^{33} \mathrm{ergs} \mathrm{s}^{-1}$ toward the ionizing luminosity, assuming a distance of $\sim 415 \mathrm{pc}$. The actual X-ray contribution could be higher if many deeply embedded sources within the cloud have escaped detection because of very high extinction. But, the above estimate of $\mathrm{L}_{\mathrm{x}, \mathrm{tot}}$ is much less than the Lyman continuum luminosity of a a B0 - O9 star (Thompson 1984) and we conclude that the presently known $\mathrm{X}$-ray population cannot by itself account for the required ionizing radiation.

Recently, it has been suggested by Bik et al. (2003) that the infrared source IRS 2b is the long-sought ionizing source. As shown in Figure 2, this source lies $\approx 5^{\prime \prime}$ west of B89-IRS 2 at P.A. $\approx-76^{\circ}$ (Jiang, Perrier, \& Léna 1984; Nisini et al. 1994). Bik et al. derive a spectral type of $\mathrm{O} 8 \mathrm{~V}-\mathrm{B} 2 \mathrm{~V}$ for IRS 2b. IRS $2 \mathrm{~b}$ was detected by Chandra (source 182) and its X-ray emission may be variable $\left(\mathrm{KS}=1.41, \mathrm{P}_{\text {var }}=0.96\right)$. Due to possible contamination from the nearby source 183 (see below), spectral fit results are somewhat uncertain. The absorption column density determined from $1 \mathrm{~T}$ VAPEC models gives $\mathrm{A}_{\mathrm{V}}=14.9[12.6-18.9 ; 90 \%$ conf.] mag using the Gorenstein (1975) conversion, but 2T models converge to an intrinsically softer spectrum with higher absorption (Table 1).

Interestingly, the Chandra image reveals two nearby X-ray sources located $\approx 1.7^{\prime \prime}-2.9^{\prime \prime}$ north of IRS 2b (Fig. 2). These are the faint X-ray source 180 and the brighter source 183, the latter being hard and variable. Interestingly, neither of these two X-ray sources have known IR counterparts, but source 183 has been detected at $3.6 \mathrm{~cm}$ in recent VLA observations (Rodriguez et al. 2003). The variability in source 183 suggests that it is an embedded young star and higher sensitive high-resolution searches for an IR counterpart are warranted.

\subsection{Interesting X-ray Sources}

Three Chandra sources showed exceptional variability that deserves further comment, namely sources 94, 192 and 207. 


\subsubsection{Persistent X-ray Variability in the cTTS Haro 5-59}

Source 94 is unusual in several respects. It was categorized as a class II source (cTTS) by HLP and is one of the few X-ray detections that has an optical counterpart, being associated with the emission-line star Haro 5-59 ( $\mathrm{V}=14.5)$. Its broad-band X-ray light curve shows a slowly declining count rate for most of the observation, with an abrupt increase near the end (Fig. 7). Closer inspection reveals that the variability is much more complex than suggested by Figure 7, as demonstrated in the four-panel light curves shown in Figure 8.

Remarkably, the X-ray source is in a state of nearly continuous variability throughout the observation with significant variations $(\geq 3 \sigma)$ present in all four parameters: observed flux, $\langle\mathrm{E}\rangle, \mathrm{L}_{\mathrm{x}}$, and $\mathrm{kT}$. The observed flux and $\mathrm{L}_{\mathrm{x}}$ generally undergo a slow decline which is punctuated by abrupt increases near UT $\approx 13 \mathrm{~h}$ and again about one hour prior to the end of the observation. The increases in flux and $\mathrm{L}_{x}$ are accompanied by increases in mean photon energy $\langle\mathrm{E}\rangle$ and $\mathrm{kT}$. These fluctuations suggest reheating of the flaring plasma during the decay, but we cannot rule out the possibility that the fluctuations are due to flaring of different plasma structures - perhaps within the same active region.

Figure 8 shows that the plasma temperature fluctuates from a low of $\mathrm{kT} \sim 2 \mathrm{keV}$ up to $\mathrm{kT} \sim 5 \mathrm{keV}$, with the maximum value reached near the end of the observation. Because of these temperature variations, isothermal fits have difficulty in reproducing the time-averaged spectrum. More sophisticated fits of the time-averaged spectrum using a DEM model (C6PVMKL) show a double-peaked structure with maxima near $\sim 1 \mathrm{keV}$ and $\sim 5 \mathrm{keV}$, confirming the multi-temperature nature of the plasma.

The persistent X-ray variability in Haro 5-59 reflects strong surface magnetic activity and may be a consequence of nearly continuous low-level flaring that produces the energy input needed to maintain the plasma at temperatures above $\mathrm{kT} \sim 2 \mathrm{keV}$. This behavior is, at least qualitatively, similar to that recently detected in sensitive XMM-Newton observations of the nearby magnetically-active star Proxima Centauri (Güdel et al. 2003). The corona of Prox Cen displayed a nearly uninterrupted sequence of low-level X-ray flares, many of which were correlated with optical variability. Evidence for continuued reheating during flares has also been obtained in other active late-type stars such as Algol (Schmitt \& Favata 1999). The variability detected here in Haro 5-59 may reflect similar processes occurring in a much younger magnetically active classical $\mathrm{T}$ Tauri star. 


\subsubsection{Slow Variability}

Prominent variability that lasted several hours was detected in sources 192 and 207. The variability is characterized by slow (non-impulsive) rise phases, as shown in Figure 7. These two sources exhibited the strongest X-ray variability of all detections as measured by their respective values $\mathrm{KS}=27.4$ and $\mathrm{KS}=17.9$ (Table 1 ). Source 192 is associated with the heavily reddened 2MASS source 05414611-0154147 ( $\mathrm{J}=17.30, \mathrm{H}=14.48, \mathrm{~K}=11.42$ ). Source 207 has a faint $K=14.8$ IR counterpart (Meyer 1996).

We have analyzed the time behavior of these two sources using light curves generated from time-binned event lists and by extracting time-partitioned spectra during the outbursts. In order to mitigate pileup, the central pixel in the PSF core was excluded in the spectra extracted for source 192 near the end of the observation when its count rate exceeded $0.1 \mathrm{c}$ $\mathrm{s}^{-1}$. The two analysis approaches give consistent results, as summarized below.

The distinguishing feature of source 192 is its high temperature. Both analysis techniques indicate that the X-ray temperature reached very high values shortly after the beginning of the outburst, certainly in excess of $\mathrm{kT} \sim 6 \mathrm{keV}$ and quite possibly as high as $\mathrm{kT}$ $\sim 10 \mathrm{keV}$. The temperature remained at values above $\mathrm{kT} \sim 4-5 \mathrm{keV}$ during the four hour rise phase monitored by Chandra, and the observed X-ray flux continued to increase until the end of the observation.

A similar situation exists for source 207, as shown in Figure 9. Remarkably, its temperature remained at levels above $\mathrm{kT} \sim 5 \mathrm{keV}$ for most of the observation and may have risen to values in excess of $\mathrm{kT} \sim 10 \mathrm{keV}$ during events such as that near UT $\approx 23 \mathrm{~h}$. Spectral analysis confirms maximum temperatures of at least $\mathrm{kT} \sim 6 \mathrm{keV}$.

In summary, sources 192 and 207 exhibit strong absorption $\left(\log \mathrm{N}_{\mathrm{H}} \sim 22.6 \mathrm{~cm}^{-2}\right.$, or $\mathrm{A}_{V}$ $\sim 18 \mathrm{mag}$ ) that is accompanied by spectacular variability and very high temperatures ( $\mathrm{kT} \geq$ $5-6 \mathrm{keV}$ ). Increases in $\langle\mathrm{E}\rangle$ (hardening of the spectrum) and temperature increases appear to occur many hours after the beginning of the outburst. We have noticed similar behavior in Chandra observations of other young stars, such as the wTTS DoAr 21 in $\rho$ Ophiuchus (Gagné et al. 2003). These results suggest that continued energy input may prolong the duration of some large outbursts.

\section{Comparison of X-ray and IR Properties}

We have used JHK magnitudes from 2MASS and other IR surveys to make compar-

isons between the X-ray and IR properties of sources in NGC 2024. Figure 17 shows the 
distribution of $\mathrm{K}$ magnitudes for those Chandra detections with known IR counterparts. The $\mathrm{X}$-ray detections show a strong peak near $\mathrm{K} \approx 11-12$. There is a strong tendency to detect the brightest $\mathrm{K}$-band sources in X-rays and there is a rapid falloff in the number of X-ray detections for $\mathrm{K}>12$. Only $20 \%$ of the catalogued IR sources in the ACIS-I FoV with $\mathrm{K}>$ 12 were detected in X-rays, while $74 \%$ of the IR sources with $\mathrm{K}<12$ were detected.

The $\mathrm{J}-\mathrm{K}$ versus $\mathrm{K}$ color magnitude diagram in Figure 18 shows that Chandra detections span a broad range of $\mathrm{J}-\mathrm{K}$ colors, but no sources fainter than $\mathrm{K}=16.7$ were detected. The faintest IR source detected was source 108 in Meyer (1996) with $K=16.74$, which corresponds to Chandra source 129. Figure 18 also reveals some notable non-detections. The class I source HLP-29 ( $\mathrm{K}=9.1$ ) was not detected by Chandra, nor was the flat-spectrum source HLP-6 $(\mathrm{K}=7.6)$. But, the latter object lies in a gap between CCDs where sensitivity was reduced.

The $\mathrm{J}-\mathrm{H}$ versus $\mathrm{H}-\mathrm{K}$ color-color diagram in Figure 19 allows us to discriminate between normally reddened stars and stars with true infrared excesses (as due, for example, to circumstellar disks). The left and right dashed lines in Figure 19 show the normal reddening loci of M0V and A0V stars assuming intrinsic J-H and H-K colors from Bessell \& Brett (1988) and the reddening law from Rieke \& Lebofsky (1985). A significant number of the Chandra detections lie between the two dashed lines and are thus likely to be normally reddened stars. More interesting are the sources lying to the right of the normal reddening band. These objects have true IR excesses and several lack IR classifications but were detected by Chandra. Thus, they are good candidates for new embedded cluster members.

\section{Candidate Protostars}

The NGC 2024 region contains the class I infrared source HLP-29 and seven compact millimeter sources FIR 1-7 which may be protostellar in nature (M88; M92). These objects represent early phases of stellar evolution about which little is known at X-ray wavelengths. We have thus carefully inspected the broad-band astrometric-corrected Chandra images for any evidence of X-ray emission from these candidate protostars.

\subsection{The Class I Source HLP-29}

No significant X-ray emission was detected from the class I source HLP-29 within a circular region of radius $3^{\prime \prime}$ centered on the J2000 position given by HLP. Only one photon was detected within the $95 \%$ encircled energy region centered at the IR position, which is consistent with the expected number of background events for a $76 \mathrm{ks}$ exposure. Since 
the extinction toward HLP-29 is not well-known, any conversion of the upper limit on its Chandra count rate to an equivalent upper limit on intrinsic $\mathrm{L}_{\mathrm{x}}$ would be highly uncertain.

\subsection{The Millimeter Sources FIR 1-7}

We searched for X-ray emission within $3^{\prime \prime}$ of the millimeter positions of FIR 1-7, including the (precessed) positions of FIR 1-6 from M88, FIR-7 from M92, and FIR 2-7 from the OVRO observations of Chandler \& Carlstrom (1996). Because of the high absorption toward these dust condensations (M92), any X-ray photons that escape are likely to be hard. Thus, we used images over a slightly broader energy range of $0.5-8 \mathrm{keV}$ (rather than $0.5-7 \mathrm{keV}$ ) to search for emission in the vicinity of FIR 1-7. Even so, no significant X-ray detections were found. However, we do find weak emission near FIR-4, as shown in Figure 4. Four photons were detected within $1.2^{\prime \prime}$ of the FIR-4 position given by M88, and three of these were localized in two adjacent pixels. The emission is hard and the mean energy of the four photons is $\langle\mathrm{E}\rangle=5.9 \mathrm{keV}$. Such emission would most likely be due to a flaring source deeply embedded within the cloud or an extragalactic source seen through the cloud. However, contamination from extragalactic sources along this line-of-sight is expected to be low due to the very high column density toward the FIR-4 dust condensation (M92). No more than one of the four photons is attributable to quiescent background in a $76 \mathrm{ks}$ exposure (Table 6.6 of $P O G$ ). Furthermore, the probability of detecting two background photons in the same pixel is no larger than $p \approx 2.6 \times 10^{-4}$, assuming a maximum quiescent background rate in the $0.5-8 \mathrm{keV}$ range of $\approx 0.22$ counts $\mathrm{s}^{-1}$ per ACIS-I CCD. The photon arrivals are well separated in time so the weak emission is not due to a cosmic ray afterglow event. The proximity of the hard X-ray emission to the FIR-4 millimeter peak and the unusual nature of this dust condensation warrant further comment.

FIR-4 is exceptional in that it is the only one of the seven condensations that has a near-IR source at the position of the millimeter peak. A faint K-band source was discovered by Moore \& Chandler (1989) and our inspection of 2MASS images confirms this K-band source at an offset of only $0.5^{\prime \prime}$ from the FIR-4 mm peak position of M88 (Fig. 4). The IR source was detected at H, K, and L bands by Moore \& Yamashita (1995= MY95). It is heavily reddened $(\mathrm{H}-\mathrm{K}=3.7)$ and there is a bright reflection nebula extending $\approx 10^{\prime \prime}$ to its northwest. MY95 suggested that the reflection nebula is due to scattering in a region channeled out by a molecular outflow, and noted that the IR source might even be a localized peak in the surface brightness of scattered light rather than the actual illuminating source. The redshifted lobe of an outflow has been detected with the Owens Valley millimeter array by Chandler \& Carlstrom (1996). 
Even though the IR source is nearly coincident with the FIR-4 millimeter peak, the physical relationship between the two is not yet clear. It was argued by M92 that the IR source does not lie at the center of the FIR-4 dust condensation, since it would then be obscured by an extinction $\mathrm{A}_{V} \sim 1000 \mathrm{mag}$ and be undetectable in the near-IR. The extinction of the IR source was estimated to be $A_{V} \leq 50$ mag by MY95. Similarly, any physical connection between the weak X-ray emission and either FIR-4 or the reddened IR source is not yet established since the X-ray peak is displaced $\approx 1^{\prime \prime}$ to the north (Fig. 4).

\section{Summary}

The embedded infrared cluster in NGC 2024 provides an interesting contrast to other well-studied star-forming regions in Orion such as the Orion Nebula Cluster (ONC). NGC 2024 lies at approximately the same distance as the ONC but has a lower stellar density and is more heavily obscured, with a dearth of optically visible stars. Although star-formation appears to have already ceased in the ONC (Feigelson et al. 2002), active star-formation may still be underway in NGC 2024 and many of the cluster members are still in the class II accretion phase.

The catalog of 283 Chandra sources is large enough for statistical studies and provides new information on the distribution of young stars within the cloud, absorption, X-ray spectral and variability properties, and energetics of the X-ray emitting population. The most important results to emerge from this study are the following:

1. Chandra detected 283 sources in NGC2024 within a $\approx 17^{\prime} \times 17^{\prime}$ ACIS-I region centered near the millimeter source FIR-5, a five-fold increase over previous X-ray surveys. A total of 248 X-ray sources (88\%) were identified with counterparts, mostly infrared sources.

2. Roughly half of the 35 sources without counterparts are expected to be extragalactic based on source counts from Chandra Deep Field surveys. About a dozen of the unidentified sources have positions within the projected boundary of the known IR cluster and some of these could be embedded young stars. We have provided accurate $\mathrm{X}$-ray positions based on registration of Chandra images with the 2MASS data base (Table 1) that will facilitate future searches for counterparts to these X-ray sources in the infrared and other spectral regions.

3. The X-ray sources in NGC 2024 are in general characterized by hard heavily absorbed emission with strong variability. By fitting the spectra of more than 100 of the brightest 
detections, we have derived a mean X-ray energy $\langle\mathrm{kT}\rangle=2.8 \mathrm{keV}$ and mean extinction $\left\langle\mathrm{A}_{V}\right\rangle=10.5 \mathrm{mag}$. This latter value is in excellent agreement with extinction estimates from IR studies. However, considerable variation about these mean values was observed, with $\mathrm{kT} \sim 6-10 \mathrm{keV}$ derived for some strongly-variable sources and extinctions as large as $\mathrm{A}_{V} \sim 68$ mag for deeply embedded sources.

4. Variability was detected at high confidence levels in $25 \%$ of the X-ray sources, but we have argued that the true variability fraction is likely to be larger because of the difficulty in detecting variability in weak sources. We find no compelling evidence that variability and spectral hardness are correlated. Of particular interest are sources which undergo poorly-understood slow variability lasting many hours during which plasma temperatures are maintained at high values $\mathrm{kT} \geq 5-6 \mathrm{keV}$ such as sources 192 and 207 (Fig. 7). Such behavior is decidedly different from solar-like impulsive bursts (e.g. sources 27, 81 and 174) that signal magnetic reconnection and rapid energy release. Our time-resolved spectroscopic analysis suggests that the plasma in such slowly varying sources is being reheated. However, we cannot spatially resolve individual flaring regions so it is possible that multiple unresolved flares are taking place, perhaps in the same active region. We also find clear evidence for persistent low-level variability in the star Haro 5-59, which is probably the consequence of nearly continuous low-level flaring in this magnetically active cTTS.

5. A comparison with IR data shows that the brightest K-band sources are preferentially detected in X-rays. Chandra detected $74 \%$ of the known IR sources with $\mathrm{K}<12$ but only $20 \%$ of those with $\mathrm{K}>12$. Near-infrared color-color diagrams reveal that many Chandra detections are heavily reddened, including extreme objects such as the hard variable X-ray source 192 (= 2MASS J05414611-0154147; J-H = 2.82, H-K=3.06; Fig. 19). These heavily reddened sources could be young cluster members and follow-up observations in the mid-IR would be useful to determine if disks are still present.

6. The detection rate of 27 known class II sources (cTTS) in NGC 2024 is at least $96 \%$, and may be as high as $100 \%$. The X-ray and bolometric luminosities of these class II sources are correlated and we also find a correlation between the observed luminosities in the equally absorbed $\mathrm{J}$ band and $1.1-1.4 \mathrm{keV}$ X-ray band. A notable exception is the IR-bright cTTS HLP-2, whose X-ray emission is at least an order of magnitude lower than expected from regression fits.

7. The ionizing source of NGC 2024 has been elusive, but previous studies have determined that the required ionization flux is equivalent to that of a B0-O9 star. We estimate the total X-ray luminosity from all $283 \mathrm{X}$-ray sources in the Chandra FoV to be $\mathrm{L}_{\mathrm{x}, \mathrm{tot}}(0.5$ $-7 \mathrm{keV}) \sim 10^{33} \mathrm{ergs} \mathrm{s}^{-1}$ at an assumed distance of $\sim 415 \mathrm{pc}$. This is significantly less 
than the Lyman continuum luminosity of a B0-O9 star. Thus, the X-ray contribution from the known population cannot account for all of the missing ionizing flux. Recent work by Bik et al. (2003) suggests that the luminous IR source IRS $2 \mathrm{~b}$ is a young O8V - B2V star and may be the long-sought ionizing source. IRS $2 \mathrm{~b}$ is detected by Chandra and its X-ray emission is likely variable, possibly a sign of magnetic activity in this embedded high-mass star.

8. Close examination of the Chandra images shows no significant X-ray detections $(\geq 6$ counts) of any of the seven millimeter-bright condensations FIR 1-7 in NGC 2024. These condensations, which may be protostellar, are thus not yet sufficiently evolved to produce X-rays or any intrinsic X-ray emission has been completely absorbed by intervening material. A potentially interesting exception is FIR-4, for which we detect faint hard localized emission (4 counts) within $1.2^{\prime \prime}$ of the mm position. Previous observations have detected a heavily-reddened near-IR source within $0.5^{\prime \prime}$ of FIR-4 as well as a CO outflow, indicating that stars have recently formed and may still be forming in this region. However, the physical relationship between the FIR-4 dust condensation, near-IR source, and faint X-ray emission is not yet clear because of the small positional offsets between the millimeter, IR, and X-ray peaks (Fig. 4).

This work was supported by SAO grant GO1-2009A. This research has made use of the SIMBAD astronomical database, the Astrophysics Data System (ADS), and the ASURV statistical analysis package maintained by Penn State. We have utilized data products from the Two Micron All Sky Survey (2MASS), which is a joint project of the Univ. of Massachusetts and IPAC/CalTech, funded by NASA and NSF. We have also utilized data and software from the High Energy Astrophysics Science Archive Research Center (HEASARC), provided by NASA's Goddard Space Flight Center. We thank M. Meyer for providing JHK photometry in electronic format from his Ph.D. thesis and L. Rodriguez for providing a table of VLA radio sources in NGC 2024 prior to publication.

\section{REFERENCES}

Anders, E., \& Grevesse, N. 1989, Geochim. Cosmochim. Acta, 53, 197

Anthony-Twarog, B.J. 1982, AJ, 87, 1213

Arnaud, K.A. 1996, in Astronomical Data Analysis Software and Systems V, eds. G. Jacoby \& J. Barnes, (San Francisco: ASP), 101, 17 
Babu, G.J. \& Feigelson, E.D., 1996, Astrostatistics: Interdisciplinary Statistics (London: Chapman, \& Hall), 66

Barger, A.J. et al. 2002, AJ, 124, 1839

Barnes, P.J., Crutcher, R.M., Bieging, J.H., Storey, J.W.V., \& Willner 1989, ApJ, 342, 883

Beck, T.L., Simon, M., \& Close, L.M. 2003, ApJ, in press

Bessell, M.S. \& Brett, J.M. 1988, PASP, 100, 1134

Bik, A. et al. 2003, A\&A, in press (astro-ph/0303029)

Casanova, S., Montmerle, T., Feigelson, E.D., \& André, P. 1995, ApJ, 439, 752

Chandler, C.J. \& Carlstrom, J.E. 1996, ApJ, 466, 338

Comerón, F., Rieke, G.H., \& Rieke, M.J. 1996, ApJ, 473, 294

Cowie, L.L., Garmire, G.P., Bautz, M.W., Barger, A.J., Brandt, W.N., \& Hornschemeier, A.E. 2002, ApJ, 566, L5

Crutcher, R.M., Roberts, D.A., Troland, T.H., \& Goss, W.M. 1999, ApJ, 515, 275

Feigelson, E.D. et al. 2002, ApJ, 574, 258

Freyberg, M.J., \& Schmitt, J.H.M.M. 1995, A\&A, 296, L21

Gagné, M., Daniel, K.J., \& Skinner, S.L. 2003, ApJ, submitted

Gehrels, N. 1986, ApJ, 303, 336

Getman, K.V. Feigelson, E.D., Townsley, L., Bally, J., Lada, C.J., \& Reipurth, B. 2002, ApJ, 575, 354

Gordon, M.A. 1969, ApJ, 158, 479

Gorenstein, P. 1975, ApJ, 198, 95

Grasdalen, G.L. 1974, ApJ, 193, 373

Güdel, M., Audard, M., Skinner, S.L., \& Horvath, M.I. 2003, ApJ, in press

Haisch, K.E., Lada, E.A., Pina, R.K., Telesco, C.M., \& Lada, C.L. 2001, AJ, 121, 1512 (HLPTL) 
Haisch, K.E., Lada, E.A., \& Lada, C.J. 2000, AJ, 120, 1396 (HLL)

Hjellming, R.M. 1968, ApJ, 154, 533

Jiang, D.R., Perrier, C., \& Léna, P. 1984, A\&A, 135, 249

Lada, E.A., Evans, N.J., Depoy, D.L., \& Gatley, I. 1991, ApJ, 371, 171

Lai, S.-P., Crutcher, R.M., Girart, J.M., \& Rao, R. 2002, ApJ, 566, 925

Mewe, R., Gronenschild, E.H.B.M., \& van den Oord, G.H.J., 1985, A\&AS, 62, 197

Meyer, M. 1996, Ph.D. Thesis, Univ. of Massachusetts (Amherst)

Mezger, P.G., Chini, R., Kreysa, E., Wink, J.E., \& Salter, C.J. 1988, A\&A, 191, 44 (M88)

Mezger, P.G., Sievers, A.W., Haslam, C.G.T., Kreysa, E., Lemke, R., Mauersberger, R., \& Wilson, T.L. 1992, A\&A, 256, 631 (M92)

Moore, T.J.T. \& Chandler, C.J. 1989, MNRAS, 241, 19p

Moore, T.J.T., Chandler, C.J., Gear, W.K., \& Mountain, C.M. 1989, MNRAS, 237, 1p

Moore, T.J.T. \& Yamashita, T. 1995, ApJ, 440, 722 (MY95)

Morrison, R. \& McCammon, D. 1983, ApJ, 270, 119

Nisini, B. et al. 1994, A\&A, 290, 463

Preibisch, T. \& Zinnecker, H. 2002, AJ, 123, 1613

Press, W.H. et al., 1992, Numerical Recipes in Fortran (New York: Cambridge U. Press), 617

Rieke, G.H. \& Lebofsky, M.J. 1985, ApJ, 288, 618

Rodriguez, L.F. et al. 2003, ApJ, in prep.

Rohatgi, V.K. 1976, An Introduction to Probability Theory and Statistics (New York: John Wiley), 539

Rosati, P., Burg., R., \& Giacconi, R., 1994, in The Soft X-ray Cosmos, AIP Conf. Proceed. no. 313 (New York: AIP), eds. E. Schlegel \& R. Petre, 26

Schmitt, J.H.M.M. \& Favata, F. 1999, Nature, 401, 44 
Schulz, A., Güsten, R., Zylka, R., \& Serabyn, E. 1991, A\&A, 246, 570

Thompson, R.I. 1984, ApJ, 283, 165

Thronson, H.A. et al. 1984, ApJ, 280, 154 
Fig. 1. - Smoothed broad-band $(0.5-7 \mathrm{keV})$ Chandra ACIS-I image of the central $\approx 4^{\prime} \times$ $4^{\prime}$ region of NGC 2024 where X-ray source density is highest. Smoothing was done with the wavelet smoothing algorithm in the HEASARC XIMAGE software package (Rosati, Burg, \& Giacconi 1994). Source numbers corresponding to the first column of Table 1 are shown for a few of the brightest sources. Crosses mark the peak positions of the compact millimeter cores FIR 1-6 from M88 (FIR 1-6) and M92 (FIR 7). Coordinates are J2000.

Fig. 2.- Unsmoothed broad-band Chandra ACIS-I image of the region near IRS 2b (source 182), which has been proposed as the ionizing source of NGC 2024 (Bik et al. 2003). Source 183 lies $1.7^{\prime \prime}$ north of IRS $2 \mathrm{~b}$ and has no known IR counterpart but has been detected with the VLA (Rodriguez et al. 2003). Source 180 has no known counterpart. Source 187 (B89IRS 2) is a bright near-IR source with $K=4.6-5.8$. The pixel size is $0.492^{\prime \prime}$ and coordinates are J2000.

Fig. 3.- Smoothed broad-band Chandra ACIS-I image of the region in the vicinity of the millimeter cores FIR 1-7. The smoothing algorithm is the same as in Figure 1. Crosses mark the positions of cores FIR 1-6 (M88) and FIR 7 (M92). Open circles mark the positions of FIR 2-7 from the OVRO observations of Chandler \& Carlstrom (1996). None of the cores were detected in X-rays with the possible exception of weak emission naear FIR-4 (see Fig. 4). Two Chandra sources (nos. 122 and 139) in the vicinity of FIR 1 and FIR 2 are identified. Coordinates are J2000.

Fig. 4.- Unsmoothed Chandra ACIS-I image (0.5 - $8 \mathrm{keV})$ showing 4 X-ray photons within $1.2^{\prime \prime}$ of the mm source FIR 4. Pixel size is $0.492^{\prime \prime}$. Dark pixels contain two photons and light pixels contain one photon. The cross $(+)$ marks the position of the $\mathrm{mm} / \mathrm{sub}-\mathrm{mm}$ source FIR-4 from the IRTF observations of M88, and a dashed circle of radius 1" centered on FIR-4 is shown for scale. The small open circle marks the position of the $3 \mathrm{~mm}$ continuum peak of FIR-4 determined with the Owens Valley millimeter array (Chandler \& Carlstrom 1996). An asterisk is at the position of the K-band source 2MASS 05414407-0154448 first identified by Moore \& Chandler (1989). The three X-ray photons at the top of the dashed circle have energies 2.49, 7.39, and $7.77 \mathrm{keV}$ while the photon just below FIR 4 has energy $5.98 \mathrm{keV}$. Coordinates are J2000.

Fig. 5.- Positions of all 283 Chandra detections in the ACIS-I field of view (filled circles), overlaid with positions of known IR sources (open circles). The IR source positions were taken from the all-sky release of the 2MASS Point Source Catalog and other surveys (B89, B03, HLL, HLP, M96). Coordinates are J2000.

Fig. 6.- Positions of the 35 Chandra detections in the ACIS-I field of view without known counterparts at IR, optical, or radio wavelengths. Six circled sources have faint hard non- 
variable X-ray emission and may be extragalactic (AGNs). The dotted line marks the approximate boundary of the embedded IR cluster. Coordinates are J2000.

Fig. 7.- Broad-band Chandra light curves of ten sources showing interesting variability. Source numbers correspond to the first column of Table 1. The count rate of source 192 may be slightly underestimated toward the end of the observation due to moderate pileup at count rates above $0.1 \mathrm{c} \mathrm{s}^{-1}$.

Fig. 8.- Chandra light curves of variable source no. 94 (= J054138.9-015936), whose optical counterpart is the emission-line star Haro 5-59. The panels show, from top to bottom, the absorbed flux $\mathrm{f}_{\mathrm{x}}(0.5-7 \mathrm{keV})$, mean photon energy $\langle\mathrm{E}\rangle$, unabsorbed luminosity $\mathrm{L}_{\mathrm{x}}(0.5-$ $7 \mathrm{keV}$ ), and plasma temperature $\mathrm{kT}$. Error bars are $1 \sigma$. Note that the source is in a state of continuous variability during the $\sim 21$ hour observation, and the plasma may have been reheated at $\approx 13 \mathrm{~h}$ and again at $\approx 27 \mathrm{~h}$.

Fig. 9.- Chandra light curves of the hard variable source no. 207 (= J054147.4-015526) showing the same quantities as in Fig. 8. Error bars are $1 \sigma$.

Fig. 10.- Chandra ACIS-I spectra of source 85. Top panel: Data points with $1 \sigma$ error bars show the background-subtracted flare spectrum, binned to a minimum of 15 counts per bin. Dotted line is the background-subtracted pre-flare spectrum binned to a minimum of 10 counts per bin (error bars omitted for clarity). Solid line is a best-fit 1T VAPEC model of the flare spectrum using solar abundances except for iron, which was held fixed at $\mathrm{Fe}=0.3$ solar. The best-fit model of the flare spectrum converges to an absorption $\mathrm{N}_{\mathrm{H}}=1.8[1.7$ $2.0] \times 10^{22} \mathrm{~cm}^{-2}$ and $\mathrm{kT}=3.8[3.3-4.3] \mathrm{keV}$ with $\chi^{2} / \mathrm{dof}=85.8 / 104$, where brackets enclose $90 \%$ confidence intervals. The pre-flare temperature is $\mathrm{kT} \approx 3.0 \mathrm{keV}$ and the observed flux increased by about a factor of two during the flare. Bottom panel: Residuals of the best-fit 1T VAPEC flare model, in the sense of data - model.

Fig. 11. - Histogram showing the fraction of variable sources $(\mathrm{KS}>1.7)$ as a function of the number of source counts. The apparent monotonic increase in variability fraction with source counts is very likely a selection effect caused by the inherent difficulties in detecting variability in weak sources.

Fig. 12.- Histogram showing number of X-ray sources as a function of mean photon energy $\langle\mathrm{E}\rangle$. The distribution is truncated at low energies below $\approx 1.4 \mathrm{keV}$ due to the absorption of soft photons by intervening cloud material. The mean for all detections is $\langle\mathrm{E}\rangle=2.7 \mathrm{keV}$.

Fig. 13.- Distribution of mean photon energy $\langle\mathrm{E}\rangle$ versus the KS variability statistic for all 283 X-ray detections in NGC 2024. Variable sources have KS $>1.7$ and lie above the 
solid line. The vertical dashed line is the average value $\langle\mathrm{E}\rangle=2.7 \mathrm{keV}$ for the entire sample. Most Chandra detections without counterparts (open squares) are non-variable and have above-average $\langle\mathrm{E}\rangle$. All four quadrants are well-populated and there is no obvious correlation between KS and $\langle\mathrm{E}\rangle$.

Fig. 14.- Absorption column density $\mathrm{N}_{\mathrm{H}}$ versus time-averaged plasma temperature $\mathrm{kT}$ as determined from $1 \mathrm{~T}$ VAPEC fits of the spectra of the brightest Chandra detections. For clarity, error bars (90\% confidence) are shown only for non-variable sources. Circled sources showed strong variability in their light curves. Sources with the highest plasma temperatures and largest absorptions are all variable. But, the converse is not true since many sources with low temperature and absorption also varied. Thus, variability alone is not a reliable indicator of temperature or absorption.

Fig. 15. - Kaplan-Meier estimator showing the cumulative X-ray luminosity distribution for known class II sources (classical T Tauri stars) in NGC 2024. The hard tail above $\log \mathrm{L}_{\mathrm{x}} \approx$ $30.7 \mathrm{ergs} \mathrm{s}^{-1}$ is due to the contribution of four class II sources that experienced rapid-onset flares or other strong variability (sources 41, 94, 133, 174).

Fig. 16.- Absorbed X-ray luminosity $(1.1-1.4 \mathrm{keV})$ versus observed J magnitude for known class II sources in NGC 2024. No extinction corrections have been applied. In this energy range the X-ray and J-band extinctions are approximately equal. The source shown as an X-ray upper limit is HLP-100, which was possibly detected (see text). Four class II sources that experienced flares or other strong variability are omitted in this figure (sources 41, 94, 133, 174).

Fig. 17.- Histogram showing the number of Chandra detections versus $\mathrm{K}$ magnitude for those X-ray sources with known IR counterparts. The dotted line shows the corresponding histogram for all 912 known IR sources in the Chandra field-of-view.

Fig. 18. - Color-magnitude diagram for IR sources within the Chandra ACIS-I field-of-view having $\mathrm{J}$ and $\mathrm{K}$ photometry. IR sources detected by Chandra are shown with filled symbols and undetected IR sources with open symbols. Undetected IR sources in the field which lack classifications based on IR spectral index are not shown. The class I source HLP-29 (circled cross) was not detected. The undetected flat-spectrum source HLP-6 (K= 7.6) lies in a gap between CCDs where sensitivity is reduced. The faintest K-band source detected by Chandra is source 129, whose IR counterpart is source 108 of Meyer 1996 (M-108; K = 16.74). Chandra source no. 160 (= M-165) is not visible in this plot and has $\mathrm{K}=12.31$, J-K $=8.59$.

Fig. 19.- Color-color diagram for IR sources within the ACIS-I field-of-view detected by 
Chandra. Also shown are the colors of the undetected class I source HLP-29 (circled cross) and the undetected class II/flat-spectrum sources listed in HLP. Chandra detections are solid symbols and undetected IR sources are open symbols. Undetected IR sources in the field which lack classifications based on spectral index are not shown. The dotted line at lower left is the main sequence. The sloping dashed lines mark the approximate reddening band for normally reddened M0V (left) and A0V (right) stars, based on data from Bessel \& Brett (1988) and Rieke \& Lebofsky (1985). The slopes of the dashed lines match that of the $\mathrm{A}_{V}$ $=10.5$ mag reddening vector, which corresponds to the mean extinction toward NGC 2024 . Sources to the right of the band have excess reddening. The most heavily reddened Chandra detections are identified. 


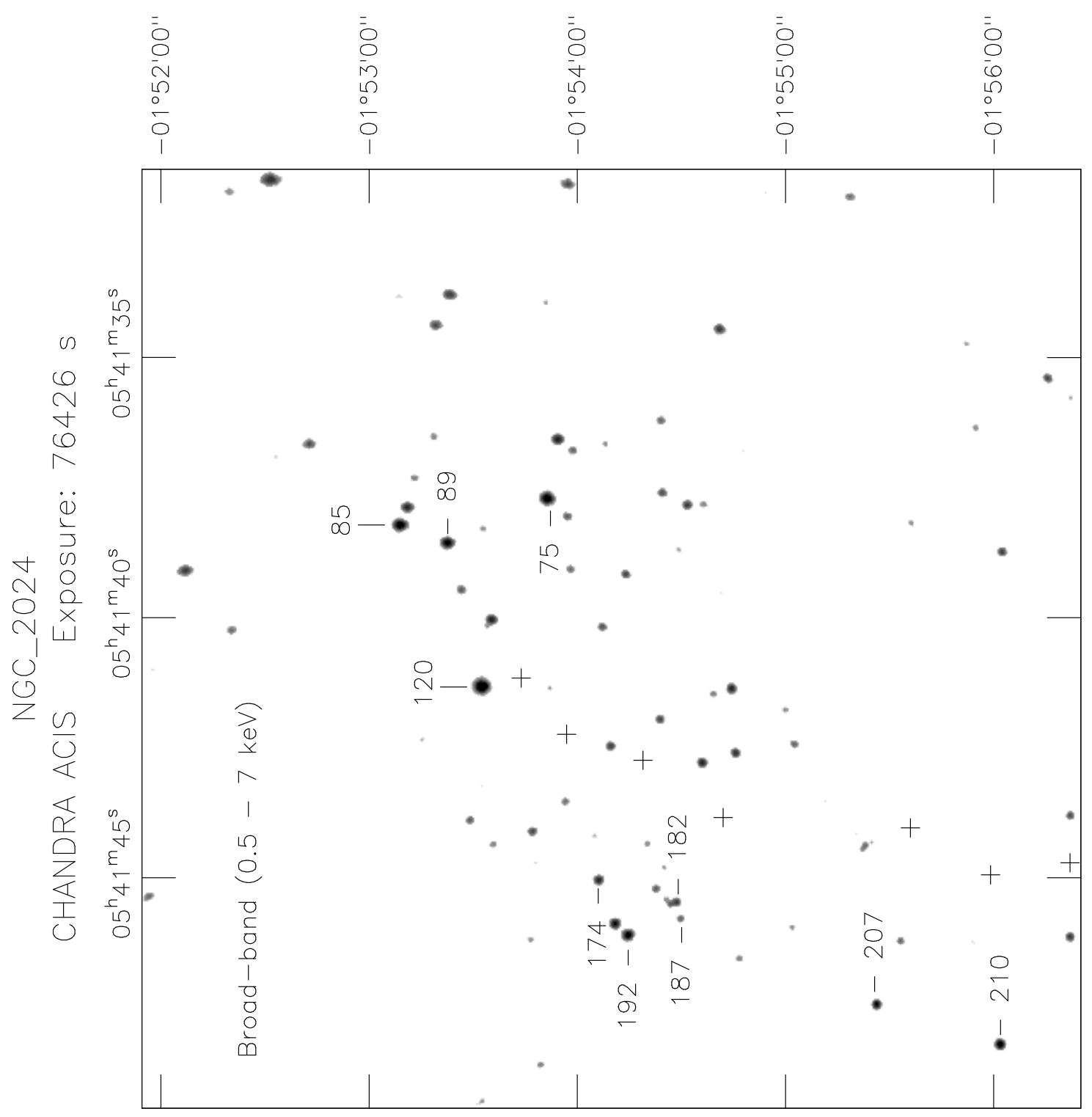

Fig. 1.- 


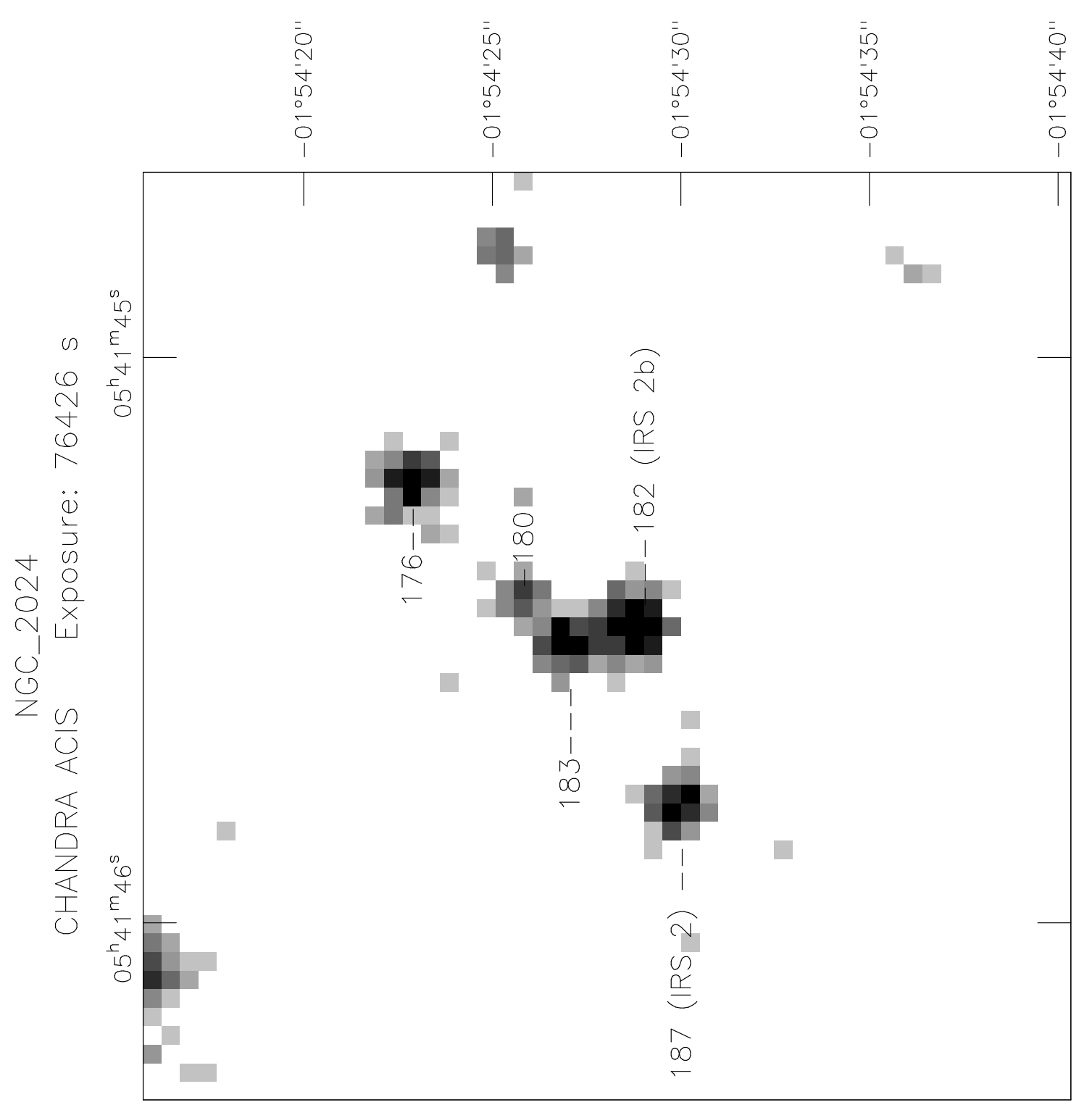

Fig. 2.- 

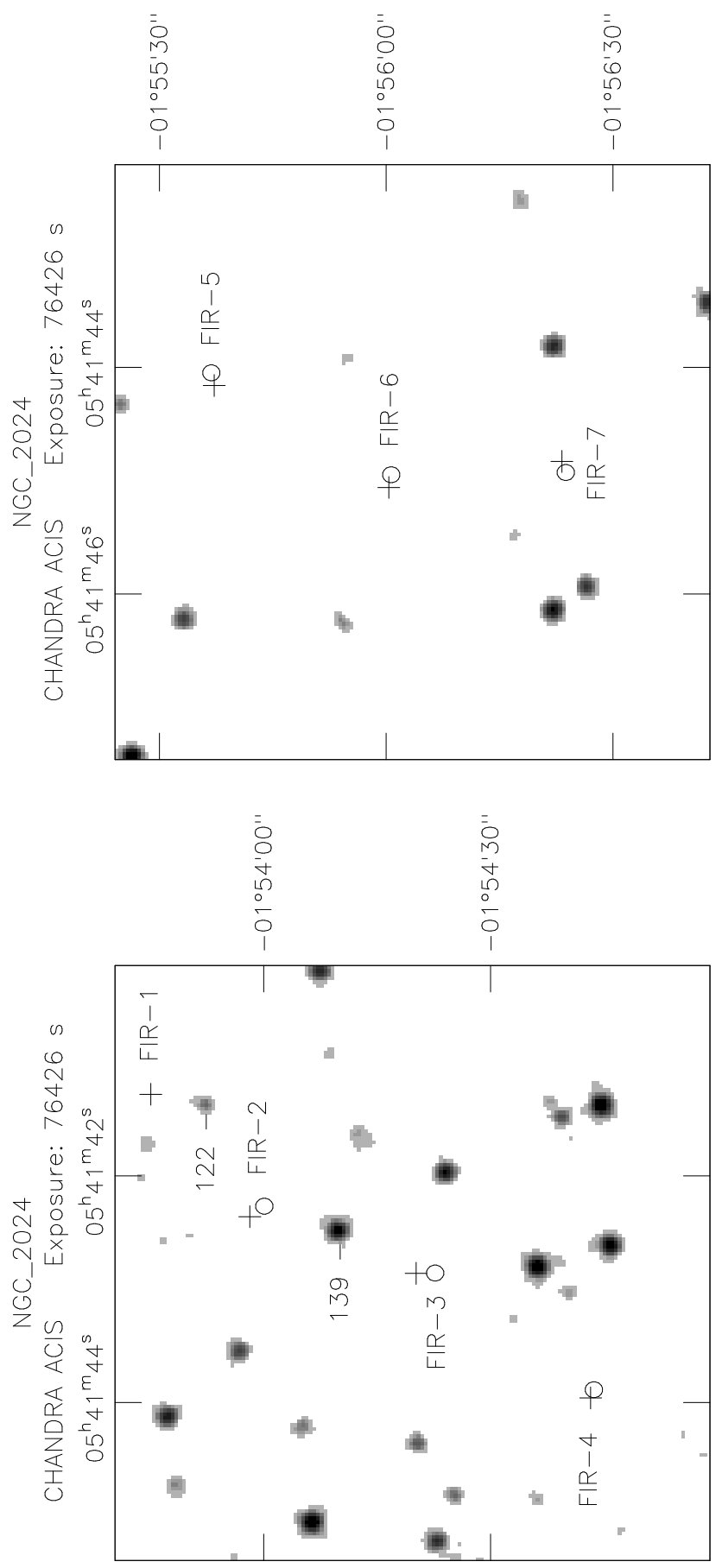

Fig. 3.- 


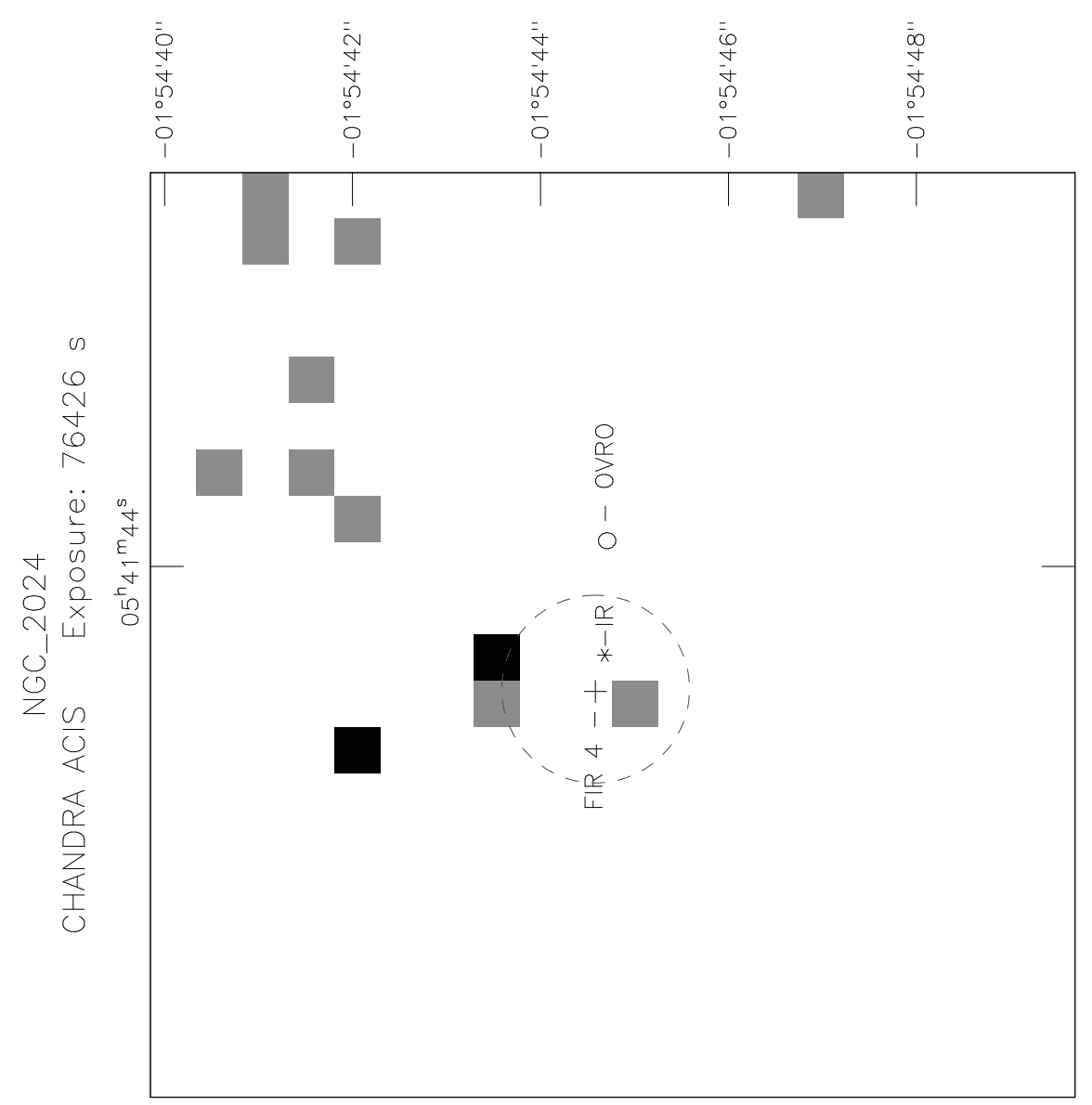

Fig. 4.- 


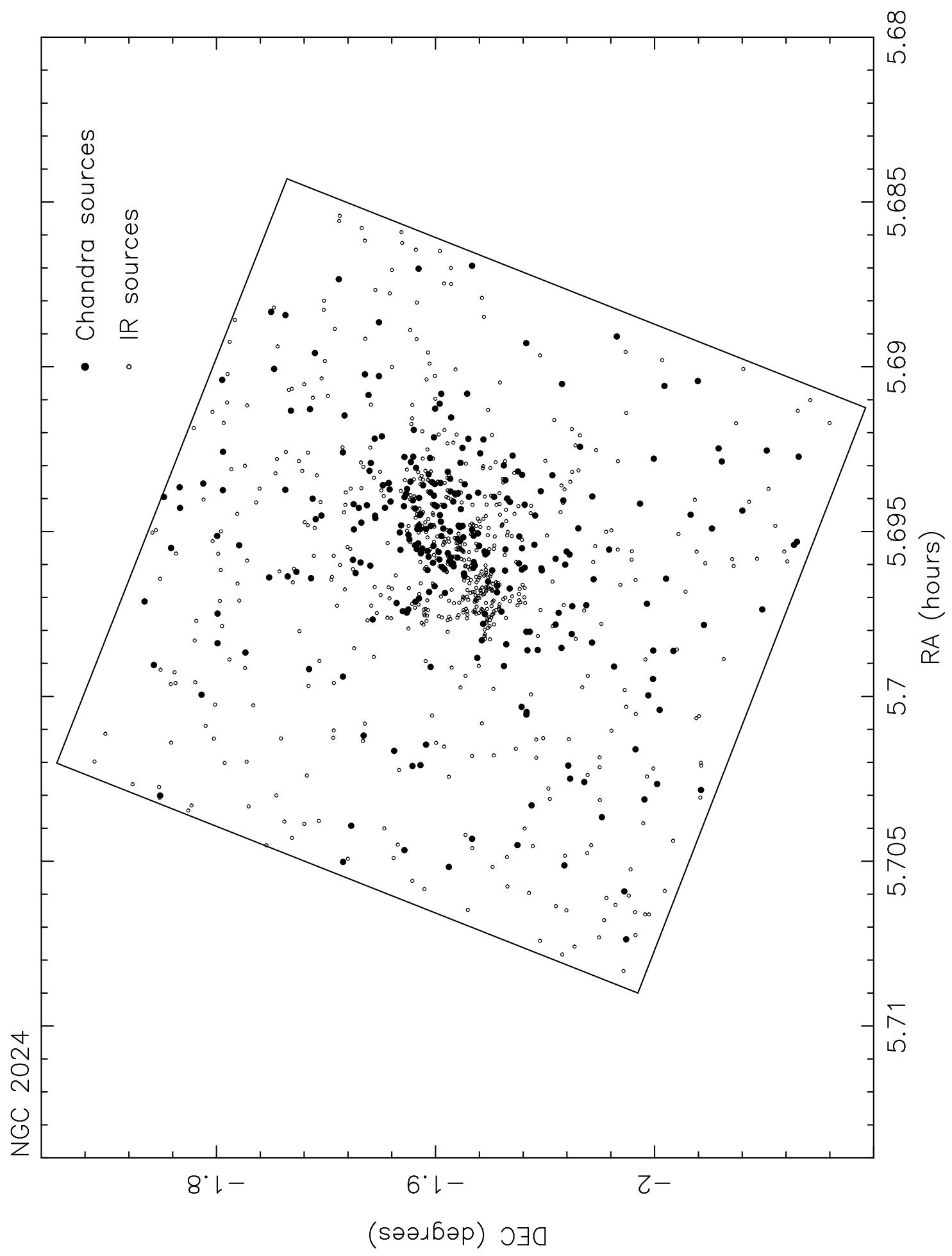

Fig. 5.- 


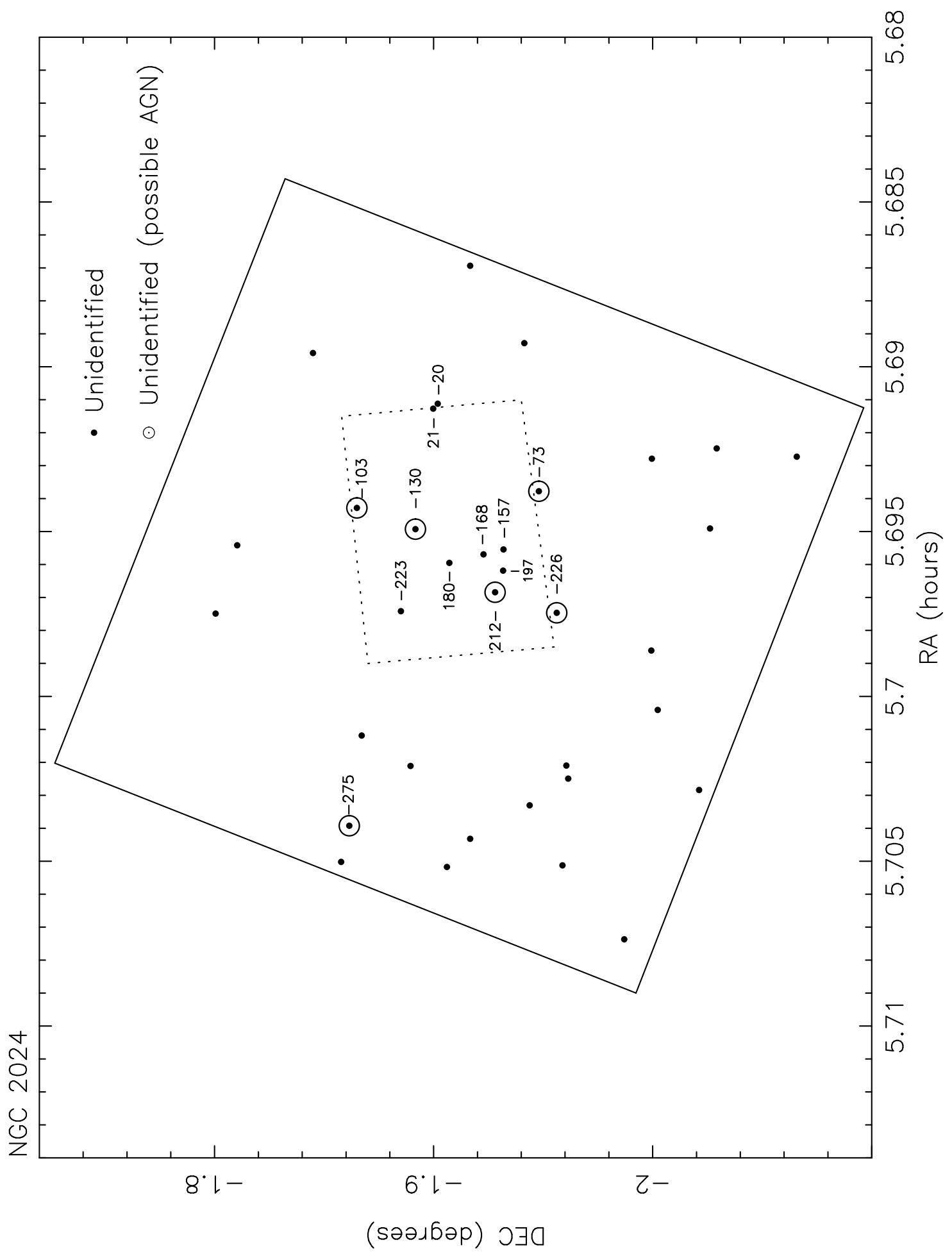

Fig. 6.- 

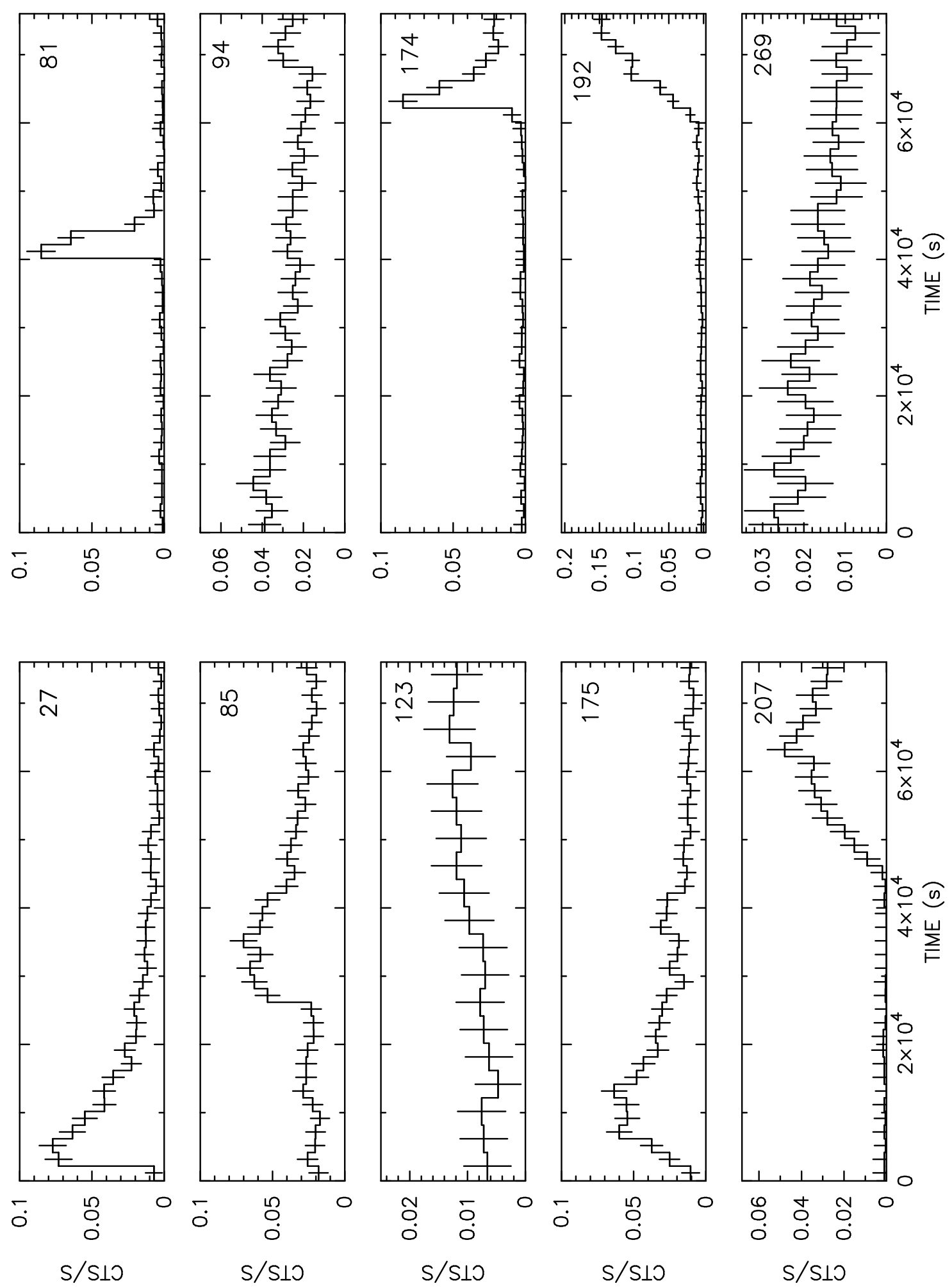

Fig. 7.- 

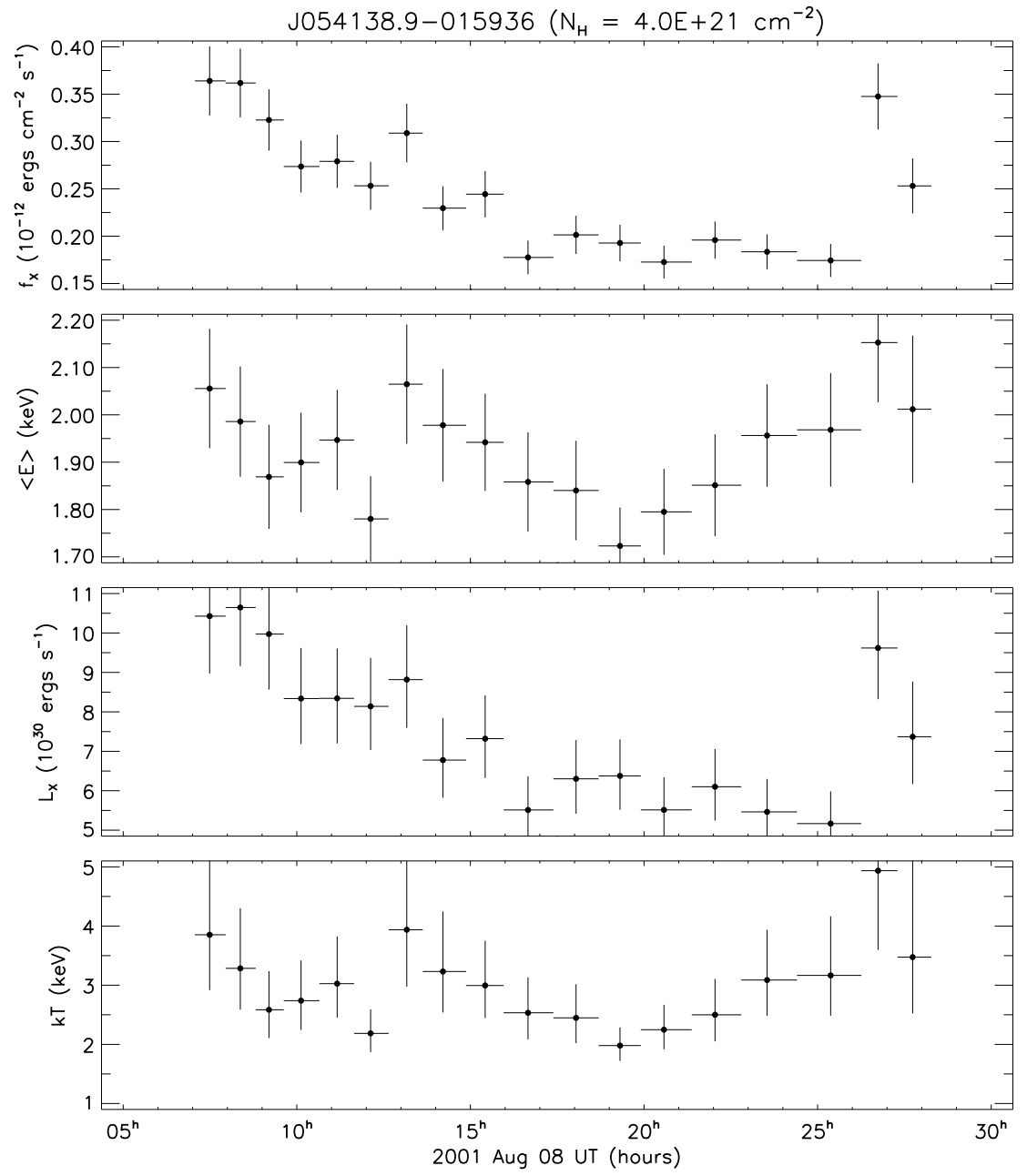

Fig. 8.- 

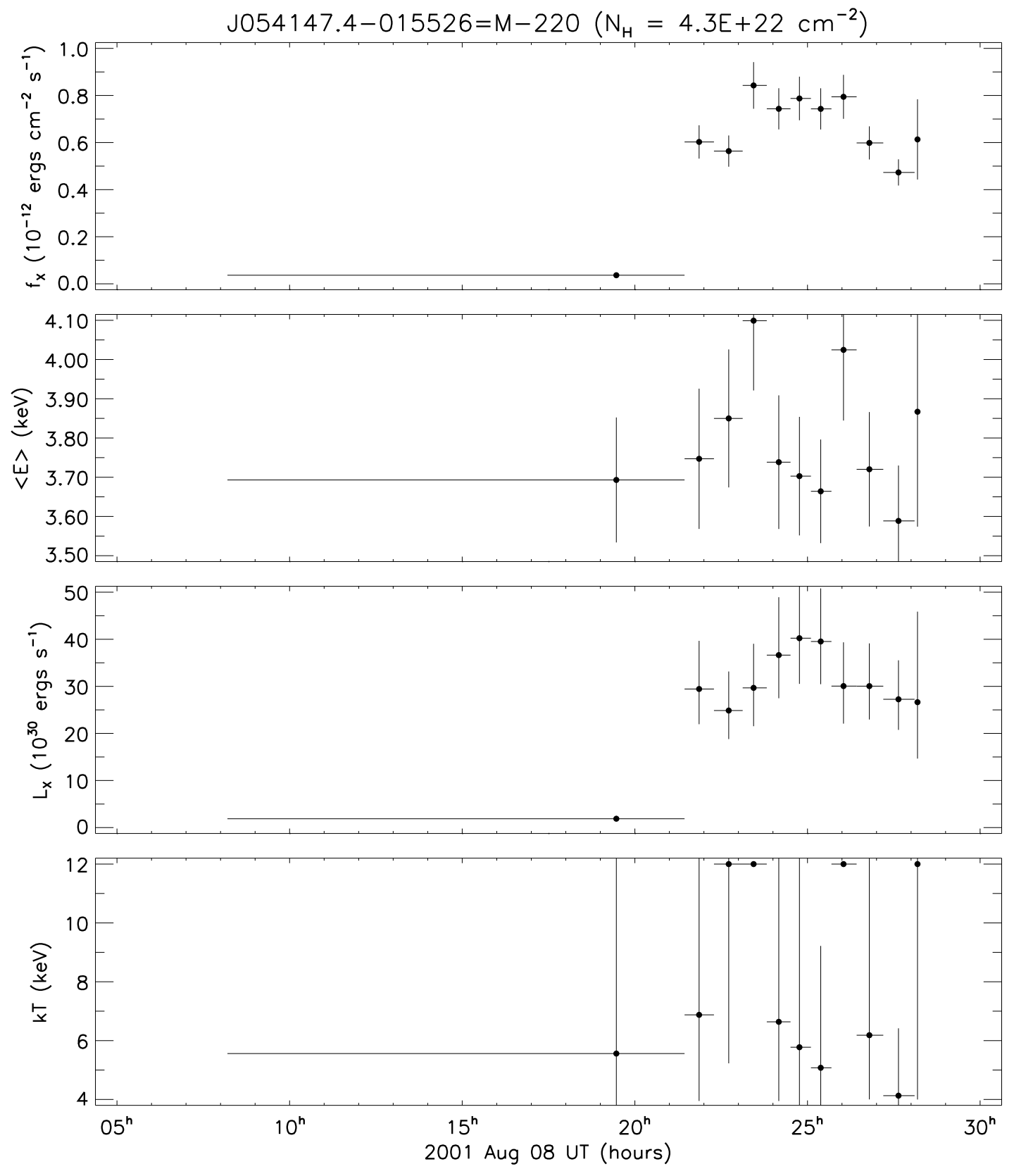

Fig. 9.- 


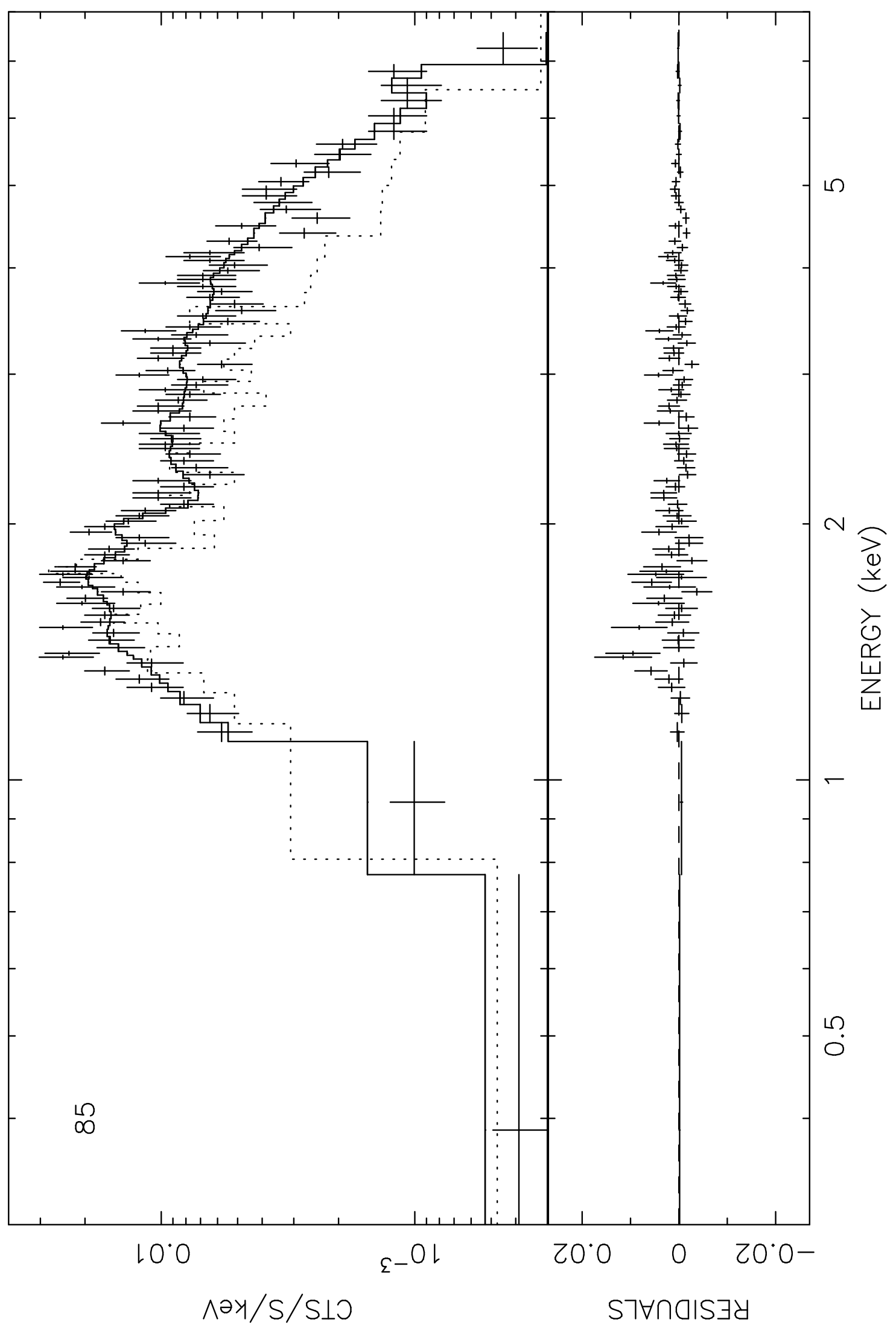

Fig. 10.- 


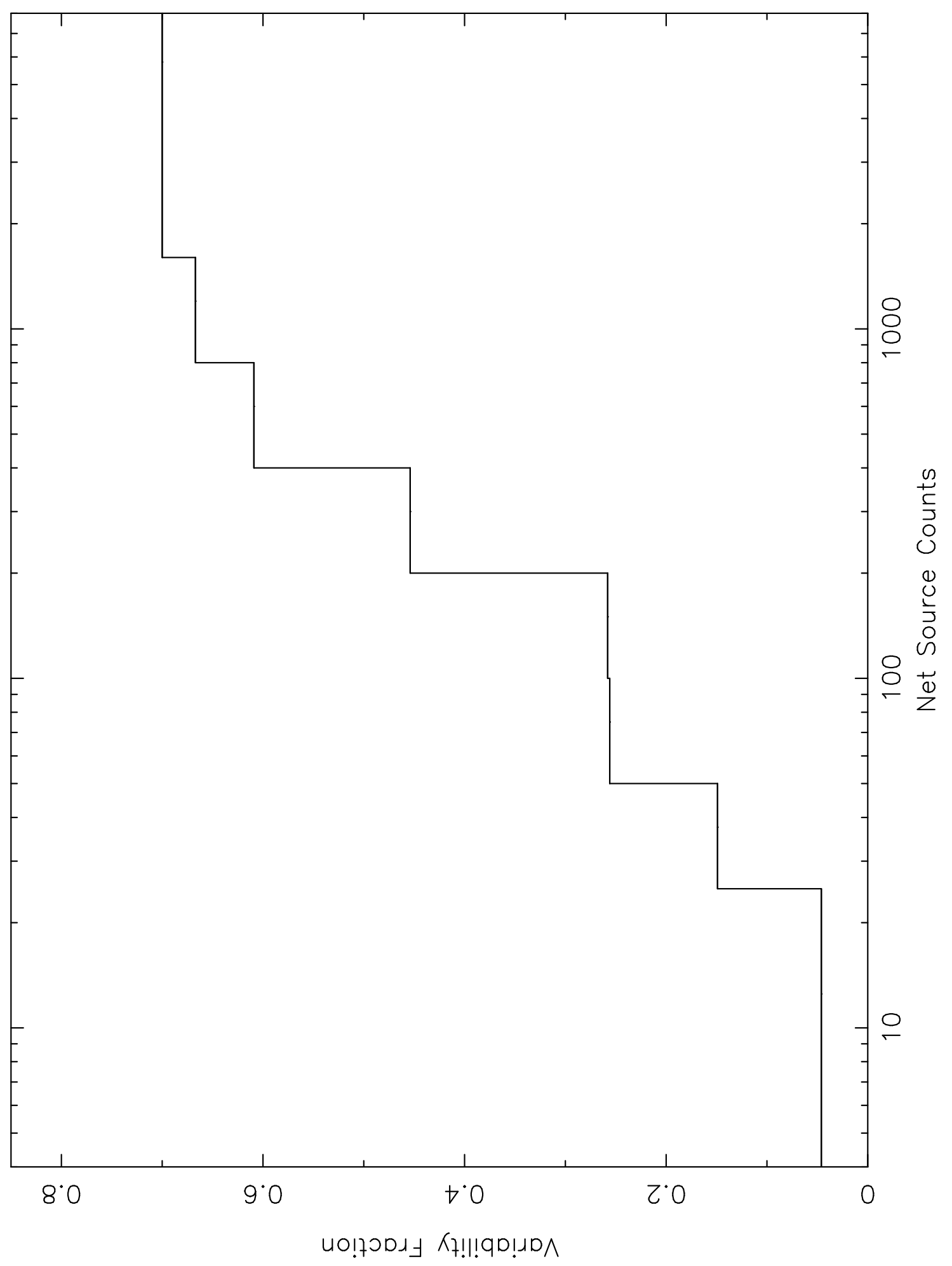

Fig. 11.- 


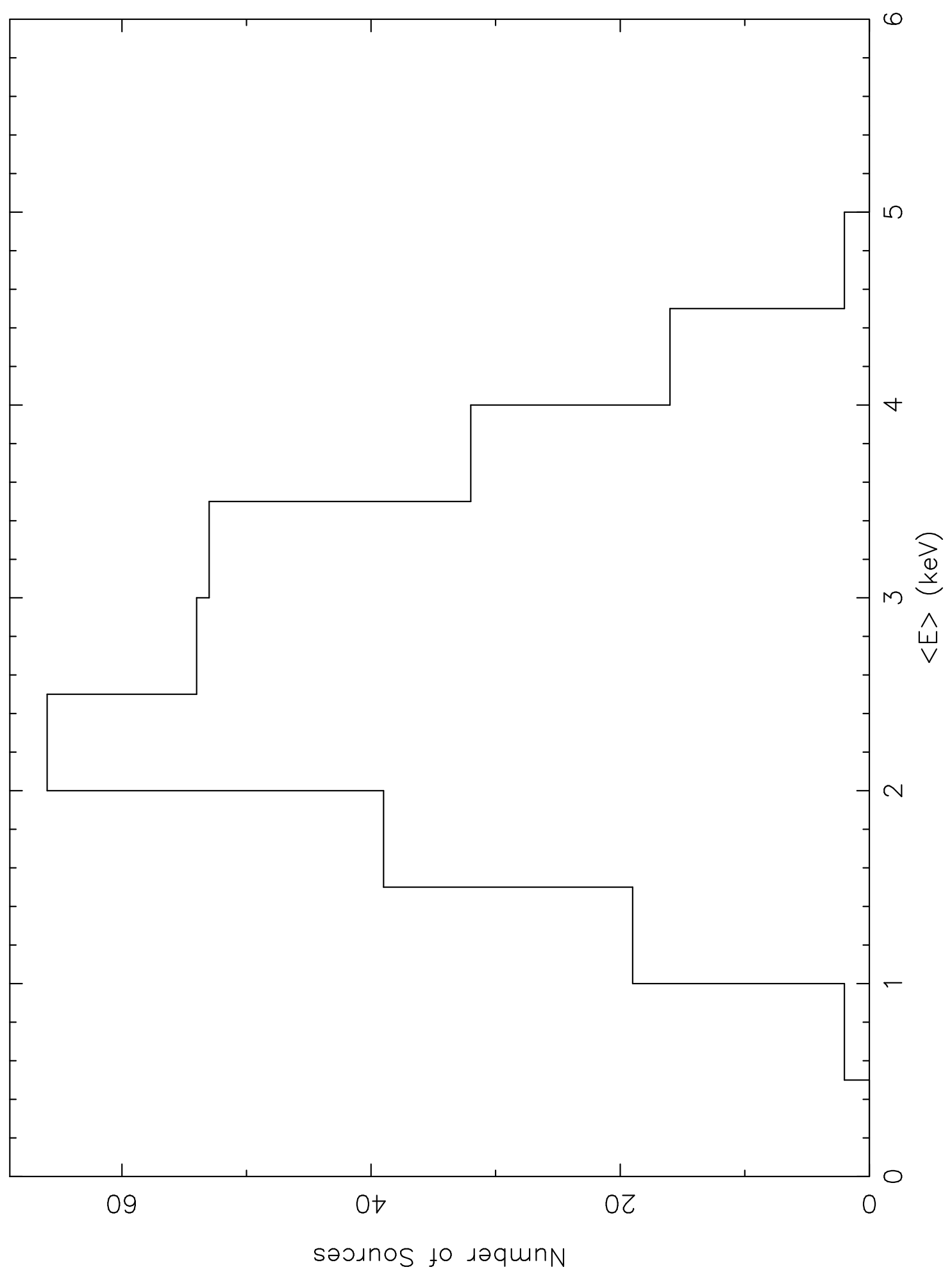

Fig. 12.- 


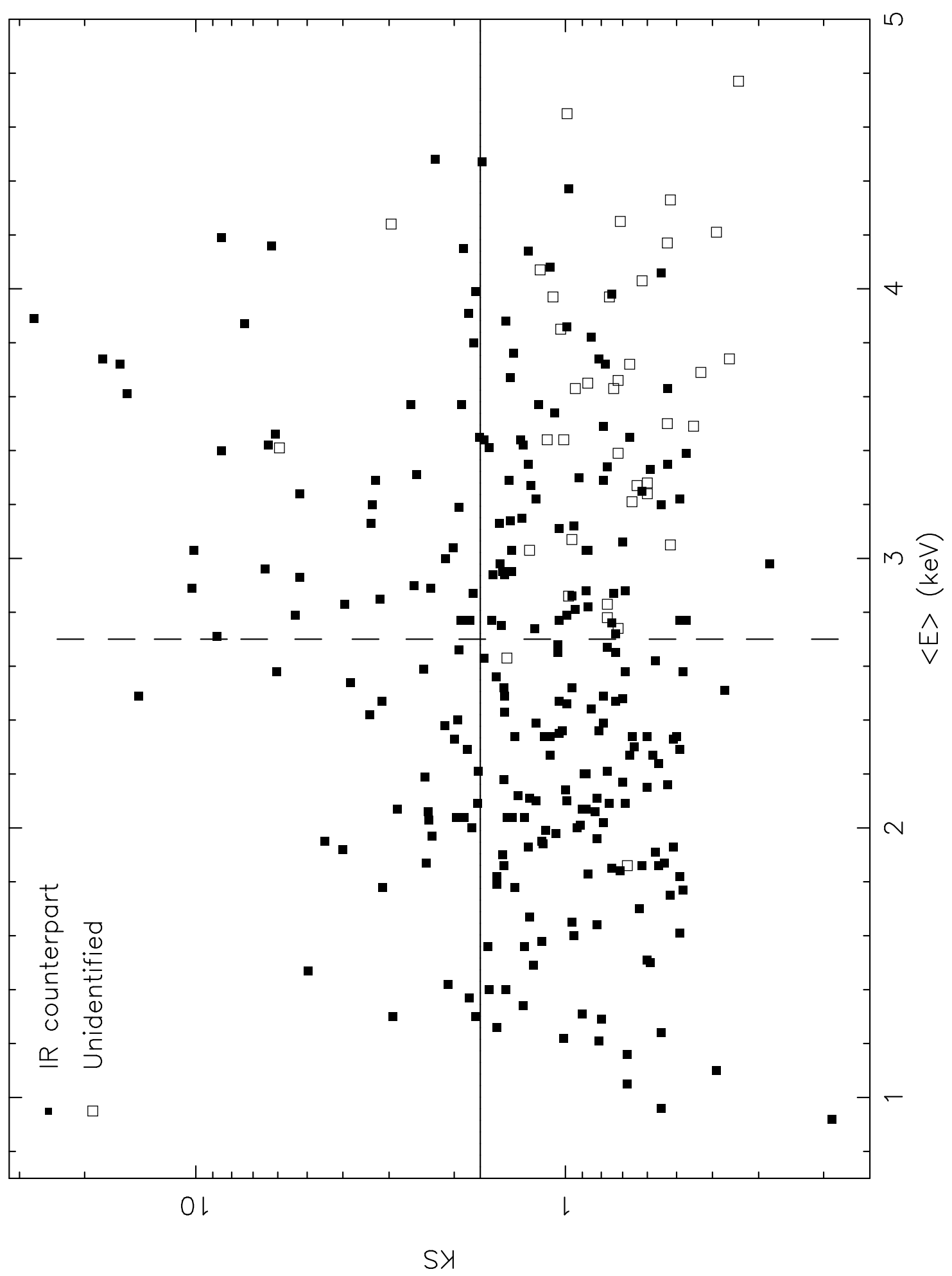

Fig. 13.- 


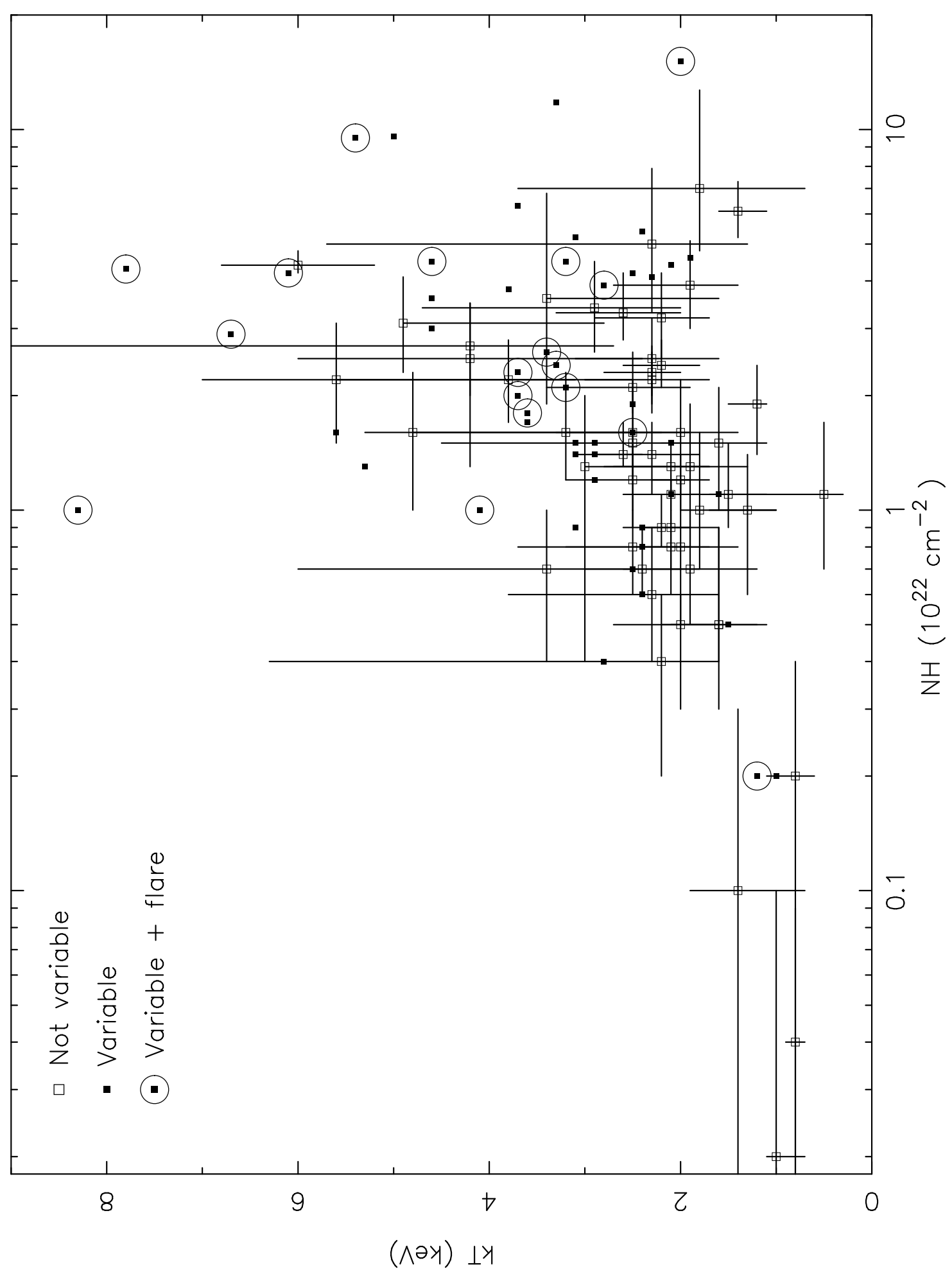

Fig. 14.- 


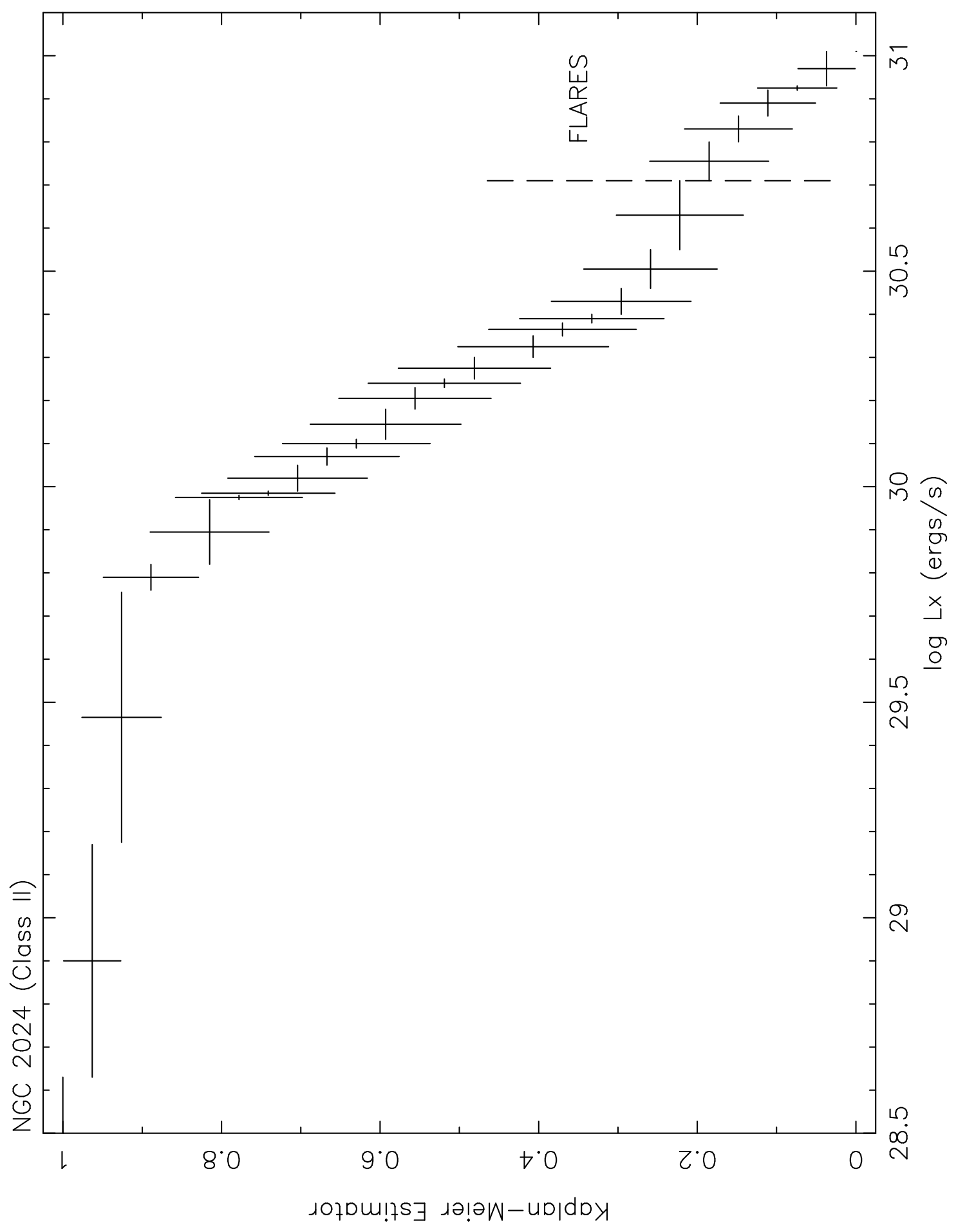

Fig. 15.- 


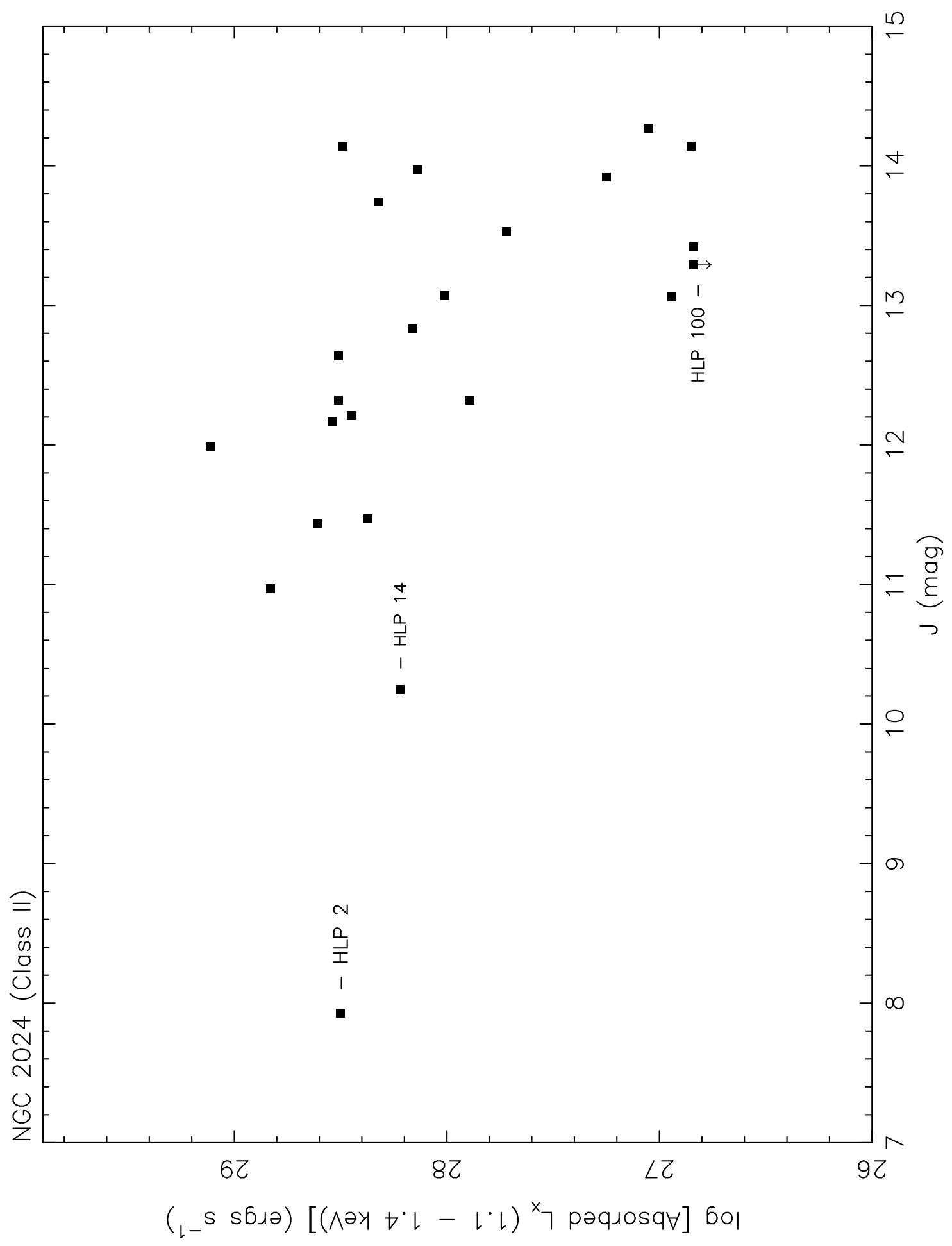

Fig. 16.- 


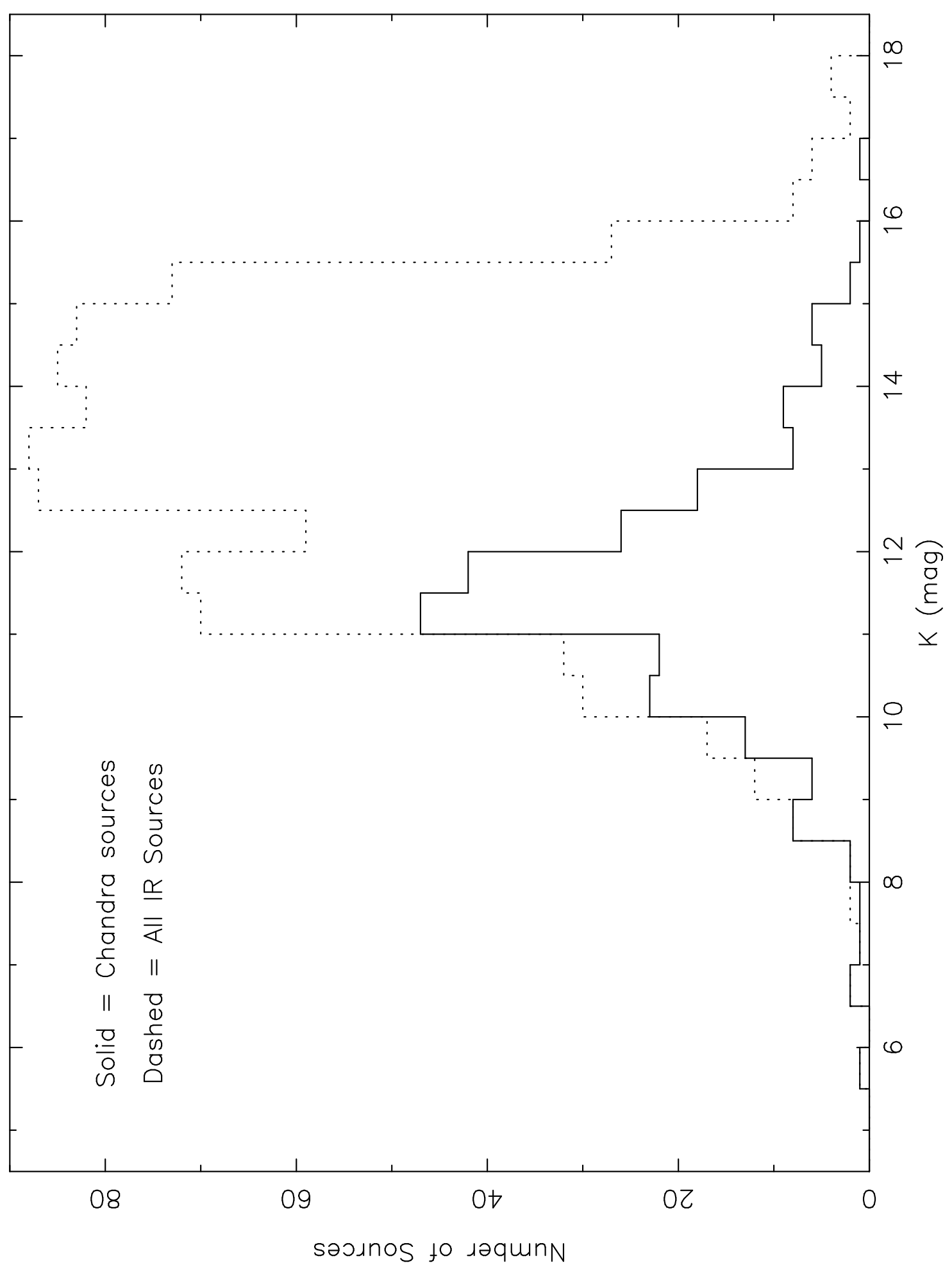

Fig. 17.- 


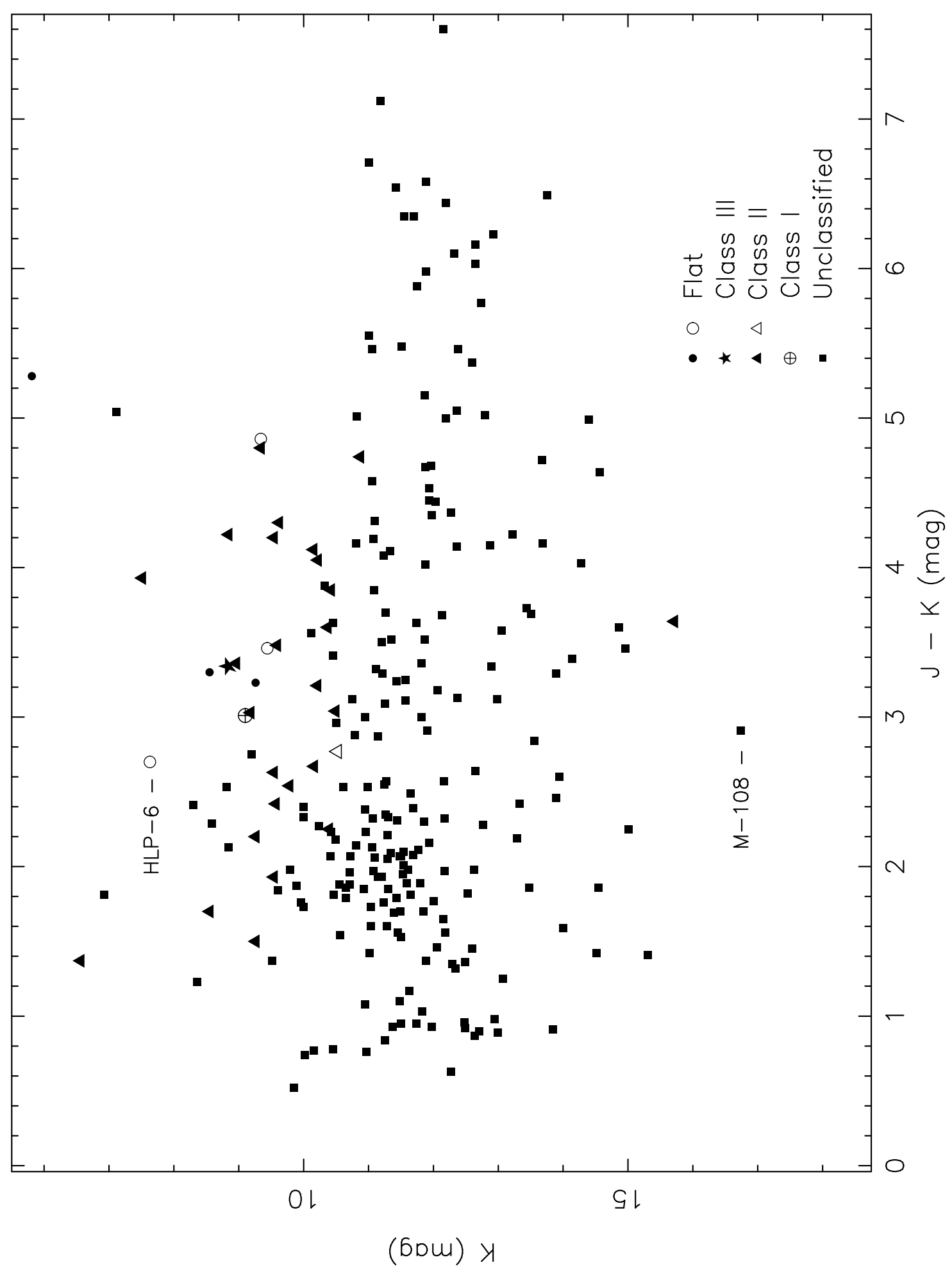

Fig. 18.- 


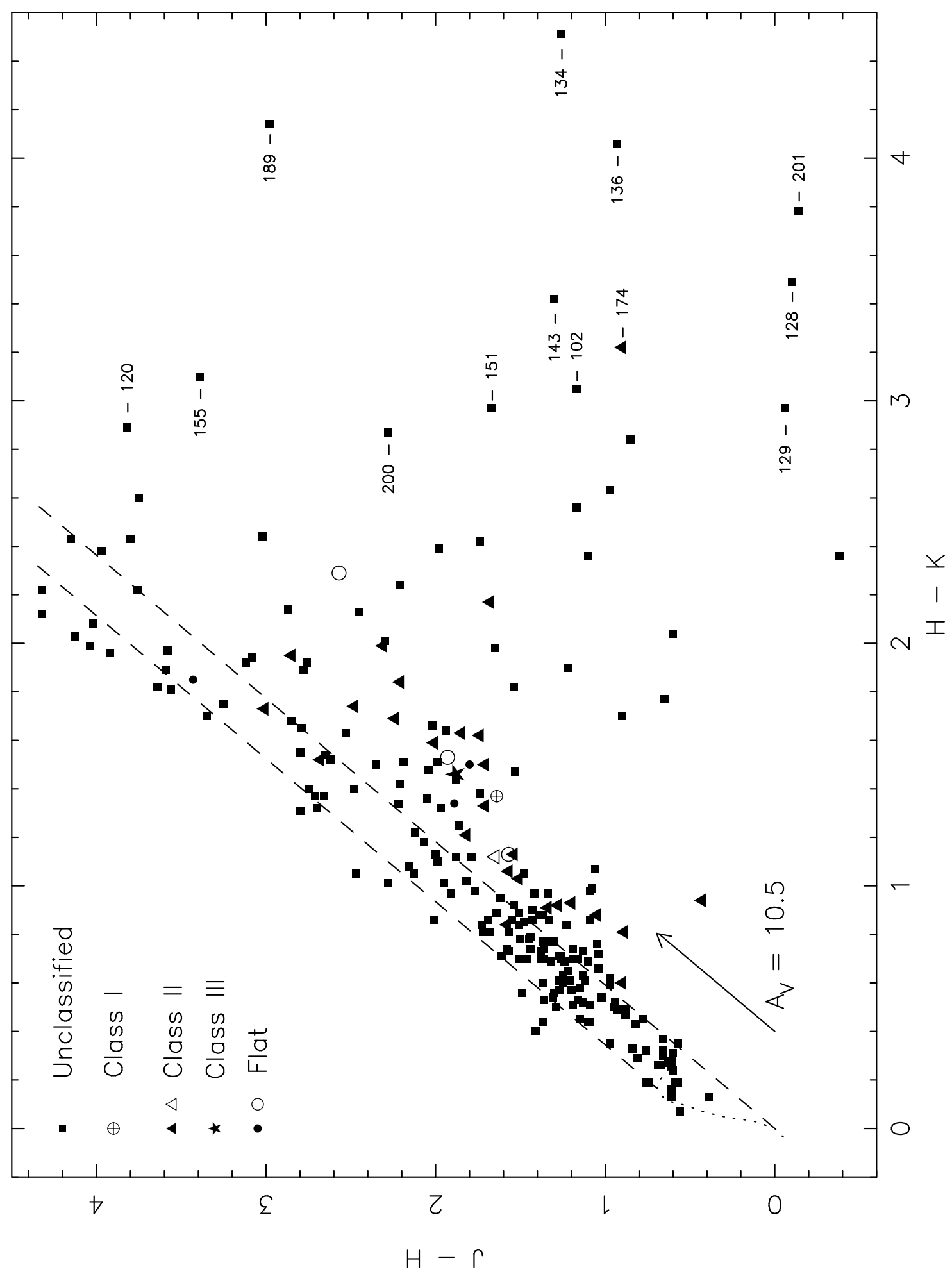

Fig. 19.- 
Table 1. Chandra X-ray Sources in NGC $2024^{\mathrm{a}}$

\begin{tabular}{|c|c|c|c|c|c|c|c|c|c|c|c|}
\hline No. & $\begin{array}{c}\text { Name } \\
(2)\end{array}$ & $\begin{array}{c}\text { R.A. } \\
\text { (J2000) } \\
(3)\end{array}$ & $\begin{array}{c}\text { Decl. } \\
(\mathrm{J} 2000) \\
(4)\end{array}$ & $\begin{array}{c}\text { Net } \\
\text { Counts } \\
(5)\end{array}$ & $\begin{array}{l}\mathrm{KS} \\
(6)\end{array}$ & $\begin{array}{c}\mathrm{E} \\
(\mathrm{keV}) \\
(7)\end{array}$ & $\begin{array}{c}\mathrm{N}_{\mathrm{H}} \\
\left(10^{22} \mathrm{~cm}^{-2}\right) \\
(8)\end{array}$ & $\begin{array}{c}\mathrm{kT} \\
(\mathrm{keV}) \\
(9)\end{array}$ & $\begin{array}{c}F_{\mathrm{x}, \mathrm{abs}} \\
\left(\mathrm{erg} / \mathrm{cm}^{2} / \mathrm{s}\right) \\
(10)\end{array}$ & $\begin{array}{c}\log L_{x} \\
(\mathrm{erg} / \mathrm{s}) \\
(11)\end{array}$ & $\begin{array}{l}\text { Identification } \\
\qquad(12)\end{array}$ \\
\hline 1 & J054112.9-015459 & 54112.97 & -15500.0 & $29 \pm 7$ & 1.25 & 3.03 & $\ldots$ & $\ldots$ & $5.33 \mathrm{e}-15$ & $\ldots$ & $\ldots$ \\
\hline 2 & J054113.2-015332 & 54113.29 & -15332.0 & $57 \pm 9$ & 0.80 & 1.29 & $\ldots$ & $\ldots$ & $5.33 \mathrm{e}-15$ & $\ldots$ & 05411324-0153306 \\
\hline 3 & J054114.4-015121 & 54114.43 & -15121.0 & $25 \pm 7$ & 1.00 & 2.14 & $\ldots$ & $\ldots$ & $2.97 \mathrm{e}-15$ & $\ldots$ & 05411449-0151204 \\
\hline 4 & J054118.0-014929 & 54118.00 & -14929.7 & $41 \pm 8$ & 1.21 & 2.74 & $\ldots$ & $\ldots$ & $7.79 \mathrm{e}-15$ & $\ldots$ & 05411799-0149292 \\
\hline $5^{*}$ & J054118.3-014953 & 54118.36 & -14953.2 & $120 \pm 12$ & 0.88 & 2.88 & $1.3_{-0.5}^{+0.8}$ & $\cdots$ & $2.45 \mathrm{e}-14$ & 30.21 & 05411838-0149531 \\
\hline 6 & J054119.1-015226 & 54119.14 & -15226.9 & $40 \pm 8$ & 0.93 & 2.00 & $\ldots$ & $\ldots$ & $5.09 \mathrm{e}-15$ & $\ldots$ & $05411918-0152268$ \\
\hline 7 & J054120.6-015857 & 54120.68 & -15858.0 & $545 \pm 25$ & 2.08 & 1.42 & $0.2_{-0.1}^{+0.1}$ & $1.2_{-0.3}^{+0.1}$ & $4.92 \mathrm{e}-14$ & 30.16 & Haro 5-46, 05412068-0158582 \\
\hline 8 & J054121.4-015629 & 54121.42 & -15629.0 & $17 \pm 6$ & 1.08 & 3.97 & $\ldots^{-0.1}$ & $\ldots^{-0.3}$ & $7.18 \mathrm{e}-15$ & $\ldots$ & $\ldots$ \\
\hline 9 & J054122.5-015041 & 54122.50 & -15041.8 & $41 \pm 8$ & 0.53 & 3.50 & $\ldots$ & $\ldots$ & $1.20 \mathrm{e}-14$ & $\ldots$ & $\ldots$ \\
\hline 10 & J054124.2-014934 & 54124.23 & -14934.7 & $666 \pm 27$ & 0.68 & 1.16 & $0.04_{-0.04}^{+0.05}$ & $0.8_{-0.1}^{+0.1}$ & $5.47 \mathrm{e}-14$ & 30.09 & HLL-2,05412424-0149348 \\
\hline 11 & J054124.8-015203 & 54124.83 & -15203.9 & $8 \pm 5$ & 0.81 & 3.74 & $\ldots$ & $\ldots$ & $3.76 \mathrm{e}-15$ & $\ldots$ & $05412475-0152063$ \\
\hline 12 & J054125.0-015226 & 54125.01 & -15226.9 & $76 \pm 10$ & 0.70 & 2.17 & $\ldots$ & $\ldots$ & $1.08 \mathrm{e}-14$ & $\ldots$ & HLL-31,05412501-0152267 \\
\hline 13 & J054125.4-014809 & 54125.43 & $\begin{array}{lll}-1 & 48 & 09.3\end{array}$ & $46 \pm 8$ & 0.60 & 2.34 & $\ldots$ & $\ldots$ & $5.68 \mathrm{e}-15$ & $\ldots$ & 05412553-0148092 \\
\hline 14 & J054125.5-020111 & 54125.55 & -20111.1 & $59 \pm 9$ & 0.90 & 1.31 & $\ldots$ & $\ldots$ & $3.92 \mathrm{e}-15$ & $\ldots$ & $05412562-0201116$ \\
\hline $15^{*}$ & J054125.8-015727 & 54125.87 & -15727.7 & $25 \pm 6$ & 0.69 & 2.09 & $\ldots$ & $\ldots$ & $4.00 \mathrm{e}-15$ & $\ldots$ & HLL-131,05412588-0157286 \\
\hline $16^{*}$ & J054126.1-020016 & 54126.10 & -20016.2 & $194 \pm 15$ & 1.04 & 2.35 & $1.6_{-0.6}^{+0.6}$ & $2.0_{-0.6}^{+1.2}$ & $2.98 \mathrm{e}-14$ & 30.35 & HLP-54,05412611-0200161 \\
\hline 17 & J054126.9-015451 & 54126.94 & -15451.8 & $87 \pm 10$ & 1.30 & 1.34 & $\ldots$ & $\ldots^{-0.6}$ & $6.24 \mathrm{e}-15$ & $\ldots$ & HLL-93,05412694-0154517 \\
\hline $18^{*} \mathrm{f}$ & J054126.9-015409 & 54126.96 & -15409.3 & $425 \pm 22$ & 8.51 & 4.19 & $9.5_{-2.6}^{+2.4}$ & $5.4_{-2.1}^{+\ldots}$ & $2.16 \mathrm{e}-13$ & 31.18 & HLL-72,05412695-0154093 \\
\hline 19 & J054127.0-015209 & 54127.08 & -15209.8 & $65 \pm 9$ & 2.30 & 1.97 & $\ldots$ & $\ldots$ & $7.22 \mathrm{e}-15$ & $\ldots$ & HLL-25,05412711-0152098 \\
\hline 20 & J054128.0-015406 & 54128.04 & -15406.8 & $16 \pm 5$ & 0.43 & 3.69 & $\ldots$ & $\ldots$ & $4.84 \mathrm{e}-15$ & $\ldots$ & $\ldots$ \\
\hline 21 & J054128.5-015359 & 54128.57 & -15359.3 & $9 \pm 4$ & 0.64 & 3.27 & $\ldots$ & $\ldots$ & $1.81 \mathrm{e}-15$ & $\ldots$ & $\ldots$ \\
\hline 22 & J054128.6-015033 & 54128.63 & -15033.6 & $56 \pm 9$ & 1.88 & 2.04 & $\ldots$ & $\ldots$ & $7.11 \mathrm{e}-15$ & $\ldots$ & HLL-9,05412868-0150331 \\
\hline 23 & J054128.7-015002 & 54128.79 & -15002.3 & $279 \pm 18$ & 1.44 & 2.04 & $1.1_{-0.2}^{+0.4}$ & $1.5_{-0.4}^{+0.4}$ & $3.52 \mathrm{e}-14$ & 30.29 & HLL-6,05412880-0150024 \\
\hline 24 & J054129.3-015130 & 54129.31 & -15130.5 & $60 \pm 9$ & 1.81 & 2.77 & $\ldots$ & $\ldots$ & $1.25 \mathrm{e}-14$ & $\ldots$ & HLL-16,05412932-0151305 \\
\hline 25 & J054129.5-015425 & 54129.53 & -15425.6 & $244 \pm 17$ & 1.53 & 1.82 & $0.5_{-0.1}^{+0.4}$ & $1.6_{-0.5}^{+0.5}$ & $2.63 \mathrm{e}-14$ & 30.00 & HLL-82,05412953-0154255 \\
\hline 26 & J054130.8-015324 & 54130.89 & -15324.3 & $26 \pm 6$ & 2.12 & 2.38 & $\ldots$ & $\ldots$ & $3.55 \mathrm{e}-15$ & $\ldots$ & 05413089-0153246 \\
\hline $27 *^{*}$ & J054131.6-015231 & 54131.61 & -15231.7 & $1358 \pm 38$ & 14.3 & 2.49 & $1.0_{-0.2}^{+0.1}$ & $4.1_{-0.4}^{+1.1}$ & $2.22 \mathrm{e}-13$ & 30.92 & 05413162-0152317 \\
\hline 28 & J054131.6-015357 & 54131.70 & -15357.3 & $488 \pm 23$ & 1.72 & 2.21 & $1.1_{-0.3}^{+0.2}$ & $2.1_{-0.4}^{+0.4}$ & $6.63 \mathrm{e}-14$ & 30.50 & HLL-66,05413170-0153573 \\
\hline 29 & J054131.8-015219 & 54131.85 & -15219.9 & $174 \pm 14$ & 1.15 & 1.94 & $0.7_{-0.2}^{+0.3}$ & $1.9_{-0.7}^{+0.7}$ & $1.92 \mathrm{e}-14$ & 29.90 & HLL-29,05413186-0152200 \\
\hline 30 & J054131.8-015453 & 54131.87 & -15453.9 & $46 \pm 8$ & 1.73 & 2.09 & $\ldots$ & $\ldots$ & $5.55 \mathrm{e}-15$ & $\ldots$ & HLL-95,05413186-0154537 \\
\hline 31 & J054131.9-015518 & 54131.94 & -15518.6 & $187 \pm 15$ & 0.88 & 2.20 & $0.7_{-0.3}^{+0.3}$ & $3.4_{-1.4}^{+2.6}$ & $2.52 \mathrm{e}-14$ & 29.98 & HLL-106,HLP-64, 05413194-0155186 \\
\hline 32 & J054132.7-015757 & 54132.74 & -15757.3 & $327 \pm 19$ & 0.81 & 1.21 & $0.02_{-0.02}^{+0.08}$ & $1.0_{-0.3}^{+0.1}$ & $2.64 \mathrm{e}-14$ & 29.71 & $05413275-0157574$ \\
\hline 33 & J054132.8-015444 & 54132.85 & -15444.3 & $27 \pm 6$ & 1.39 & 2.04 & $\ldots$ & $\ldots$ & $3.51 \mathrm{e}-15$ & $\ldots$ & 05413284-0154442 \\
\hline 34 & J054132.9-020145 & 54132.92 & -20145.3 & $20 \pm 6$ & 1.01 & 3.44 & $\ldots$ & $\ldots$ & $6.15 \mathrm{e}-15$ & $\ldots$ & $\ldots$ \\
\hline
\end{tabular}


Table 1-Continued

\begin{tabular}{|c|c|c|c|c|c|c|c|c|c|c|c|}
\hline No. & $\begin{array}{c}\text { Name } \\
(2)\end{array}$ & $\begin{array}{c}\text { R.A. } \\
\text { (J2000) } \\
(3)\end{array}$ & $\begin{array}{c}\text { Decl. } \\
(\mathrm{J} 2000) \\
(4)\end{array}$ & $\begin{array}{c}\text { Net } \\
\text { Counts } \\
(5)\end{array}$ & $\begin{array}{l}\mathrm{KS} \\
\text { (6) }\end{array}$ & $\begin{array}{c}\mathrm{E} \\
(\mathrm{keV}) \\
(7)\end{array}$ & $\begin{array}{c}\mathrm{N}_{\mathrm{H}} \\
\left(10^{22} \mathrm{~cm}^{-2}\right) \\
(8)\end{array}$ & $\begin{array}{c}\mathrm{kT} \\
(\mathrm{keV}) \\
(9)\end{array}$ & $\begin{array}{c}\mathrm{F}_{\mathrm{x}, \mathrm{abs}} \\
\left(\mathrm{erg} / \mathrm{cm}^{2} / \mathrm{s}\right) \\
(10)\end{array}$ & $\begin{array}{c}\log L_{x} \\
(\mathrm{erg} / \mathrm{s}) \\
(11)\end{array}$ & $\begin{array}{l}\text { Identification } \\
\qquad(12)\end{array}$ \\
\hline 35 & J054133.1-020304 & 54133.15 & -20304.4 & $32 \pm 7$ & 0.55 & 1.24 & $\ldots$ & $\ldots$ & $2.60 \mathrm{e}-15$ & $\ldots$ & 05413319-0203051 \\
\hline $36^{*}$ & J054133.2-014810 & 54133.28 & -14810.7 & $2434 \pm 51$ & 1.96 & 2.40 & $1.2_{-0.1}^{+0.4}$ & $2.9_{-0.3}^{+0.5}$ & $4.28 \mathrm{e}-13$ & 31.26 & 05413329-0148108 \\
\hline 37 & J054133.3-015127 & 54133.35 & -15127.7 & $36 \pm 7$ & 0.49 & 1.82 & $\ldots$ & $\ldots$ & $3.28 \mathrm{e}-15$ & $\ldots$ & HLL-15,05413337-0151271 \\
\hline 38 & J054133.4-015513 & 54133.46 & -15513.3 & $8 \pm 4$ & 1.04 & 2.47 & $\ldots$ & $\ldots$ & $1.73 \mathrm{e}-15$ & $\ldots$ & HLL-102,05413347-0155134 \\
\hline $39^{*}$ & J054133.6-015606 & 54133.70 & -15606.6 & $13 \pm 5$ & 1.47 & 2.52 & $\ldots$ & $\ldots$ & $3.00 \mathrm{e}-14$ & $\ldots$ & HLL-115,05413371-0156068 \\
\hline 40 & J054133.8-020356 & 54133.81 & -20356.8 & $17 \pm 6$ & 0.45 & 3.49 & $\ldots$ & $\ldots$ & $5.65 \mathrm{e}-15$ & $\ldots$ & .. \\
\hline $41 *_{\mathrm{f}}$ & J054133.8-015323 & 54133.82 & -15323.4 & $643 \pm 26$ & 3.81 & 2.54 & $1.6_{-0.2}^{+0.2}$ & $2.5_{-0.5}^{+0.6}$ & $1.02 \mathrm{e}-13$ & 30.71 & HLL-47,HLP-39,05413382-0153234 \\
\hline 42 & J054133.8-015308 & 54133.85 & -15308.8 & $61 \pm 9$ & 0.71 & 1.84 & $\ldots$ & $\ldots$ & $5.92 \mathrm{e}-15$ & $\ldots$ & HLL-38,05413386-0153087 \\
\hline 43 & J054133.9-015350 & 54133.97 & -15350.9 & $71 \pm 10$ & 0.60 & 2.15 & $\ldots$ & $\ldots$ & $8.49 \mathrm{e}-15$ & $\ldots$ & HLL-62,05413397-0153510 \\
\hline 44 & J054134.0-015958 & 54134.04 & -15958.7 & $24 \pm 6$ & 1.03 & 3.85 & $\ldots$ & $\ldots$ & $9.03 \mathrm{e}-15$ & $\ldots$ & $\ldots$ \\
\hline 45 & J054134.3-020150 & 54134.35 & -20150.4 & $1809 \pm 44$ & 1.75 & 1.30 & $0.2_{-0.1}^{+0.1}$ & $1.0_{-0.3}^{+0.3}$ & $1.41 \mathrm{e}-13$ & 30.65 & 05413436-0201507 \\
\hline 46 & J054134.4-015319 & 54134.40 & -15319.4 & $442 \pm 22$ & 1.10 & 2.27 & $1.3_{-0.2}^{+0.1}$ & $2.1_{-0.4}^{+0.6}$ & $5.83 \mathrm{e}-14$ & 30.48 & HLL-44,05413441-0153194 \\
\hline 47 & J054134.4-015441 & 54134.49 & -15441.0 & $552 \pm 25$ & 0.90 & 2.07 & $0.7_{-0.1}^{+0.2}$ & $2.4_{-0.6}^{+0.4}$ & $6.36 \mathrm{e}-14$ & 30.43 & HLL-88,05413448-0154410 \\
\hline 48 & J054134.5-015213 & 54134.51 & -15213.5 & $58 \pm 9$ & 0.82 & 1.96 & $\ldots$ & $\ldots$ & $6.12 \mathrm{e}-15$ & $\ldots$ & 05413451-0152135 \\
\hline 49 & J054134.7-015552 & 54134.76 & -15552.1 & $61 \pm 9$ & 1.77 & 3.80 & $\ldots$ & $\ldots$ & $3.43 \mathrm{e}-14$ & $\ldots$ & $05413476-0155522$ \\
\hline 50 & J054135.0-015327 & 54135.03 & -15328.0 & $21 \pm 6$ & 0.53 & 2.16 & $\ldots$ & $\ldots$ & $3.31 \mathrm{e}-15$ & $\ldots$ & 05413502-0153286,B-1,M-1 \\
\hline 51 & J054135.3-015211 & 54135.37 & -15211.4 & $6 \pm 4$ & 0.73 & 2.65 & $\ldots$ & $\ldots$ & $9.67 \mathrm{e}-16$ & $\ldots$ & HLL-24,05413537-0152116 \\
\hline $52 * \mathrm{f}$ & J054135.4-015615 & 54135.43 & -15615.5 & $315 \pm 19$ & 4.96 & 1.47 & $0.2_{-0.1}^{+0.3}$ & $1.2_{-0.3}^{+0.3}$ & $2.77 \mathrm{e}-14$ & 29.88 & M-6,05413543-0156154 \\
\hline 53 & J054135.4-015420 & 54135.47 & -15420.1 & $13 \pm 5$ & 1.75 & 3.99 & $\ldots$ & $\ldots$ & $3.70 \mathrm{e}-15$ & $\ldots$ & 05413547-0154195,B-2,M-8 \\
\hline 54 & J054135.7-015348 & 54135.74 & -15348.6 & $25 \pm 6$ & 1.71 & 3.45 & $\ldots$ & $\ldots$ & $6.35 \mathrm{e}-15$ & $\ldots$ & 05413575-0153483,B-3,M-11 \\
\hline 55 & J054135.8-015622 & 54135.80 & -15622.2 & $49 \pm 8$ & 1.31 & 3.15 & $\ldots$ & $\ldots$ & $1.09 \mathrm{e}-14$ & $\ldots$ & HLL-118,M-12,05413581-0156222 \\
\hline 56 & J054135.8-015712 & 54135.85 & -15712.1 & $238 \pm 16$ & 0.74 & 2.87 & $3.2_{-0.7}^{+1.0}$ & $2.2_{-0.5}^{+0.7}$ & $4.48 \mathrm{e}-14$ & 30.55 & HLL-126,05413585-0157122 \\
\hline 57 & J054136.2-015424 & 54136.23 & -15424.1 & $184 \pm 15$ & 1.51 & 3.13 & $2.2_{-0.7}^{+0.9}$ & $5.6_{-2.5}^{+\ldots .5}$ & $3.81 \mathrm{e}-14$ & 30.30 & HLL-81,HLP-26,05413623-0154241,M-17,B-6 \\
\hline 58 & J054136.3-015554 & 54136.38 & -15554.8 & $86 \pm 10$ & 0.62 & 1.86 & $\ldots$ & $\ldots$ & $1.74 \mathrm{e}-14$ & $\ldots$ & HLL-113,M-22,05413638-0155548 \\
\hline 59 & J054136.5-015318 & 54136.54 & -15318.8 & $114 \pm 12$ & 3.39 & 2.42 & $1.5_{-0.5}^{+0.5}$ & $2.1_{-0.7}^{+1.2}$ & $1.67 \mathrm{e}-14$ & 29.95 & HLL-43,05413654-0153188,B-12,M-25 \\
\hline 60 & J054136.5-015354 & 54136.60 & -15354.4 & $985 \pm 32$ & 0.89 & 2.20 & $1.2_{-0.1}^{+0.2}$ & $2.0_{-0.3}^{+0.3}$ & $1.24 \mathrm{e}-13$ & 30.80 & HLL-63,HLP-56,05413659-0153544,B-13,M-27 \\
\hline 61 & J054136.6-015242 & 54136.69 & -15242.9 & $413 \pm 21$ & 2.35 & 2.06 & $0.8_{-0.2}^{+0.1}$ & $2.4_{-0.5}^{+0.3}$ & $4.86 \mathrm{e}-14$ & 30.29 & HLL-33,05413669-0152432,B-16 \\
\hline 62 & J054136.6-015408 & 54136.69 & -15408.2 & $68 \pm 9$ & 1.79 & 2.00 & $\ldots$ & $\ldots$ & $7.32 \mathrm{e}-15$ & $\ldots$ & HLL-71,05413668-0154082,B-15,M-30 \\
\hline 63 & J054136.7-014738 & 54136.75 & -14738.3 & $42 \pm 8$ & 0.87 & 1.83 & $\ldots$ & $\ldots$ & $1.13 \mathrm{e}-14$ & $\ldots$ & $05413671-0147381$ \\
\hline 64 & J054136.8-015358 & 54136.81 & -15358.8 & $202 \pm 15$ & 1.34 & 2.12 & $0.9_{-0.2}^{+0.3}$ & $2.1_{-0.5}^{+0.3}$ & $2.40 \mathrm{e}-14$ & 30.05 & HLL-67,HLP-53,05413681-0153589,B-18,M-32 \\
\hline 65 & J054136.8-015447 & 54136.84 & -15447.9 & $31 \pm 7$ & 1.14 & 2.34 & $\ldots$ & $\ldots$ & $4.04 \mathrm{e}-15$ & $\ldots$ & HLL-92,05413682-0154479,B-19,M-33 \\
\hline 66 & J054136.9-015233 & 54136.93 & -15233.3 & $52 \pm 8$ & 1.48 & 1.90 & $\ldots$ & $\ldots$ & $5.24 \mathrm{e}-15$ & $\ldots$ & HLL-32,05413693-0152333,B-24 \\
\hline 67 & J054136.9-015339 & 54136.93 & -15339.5 & $21 \pm 6$ & 0.66 & 2.34 & $\ldots$ & $\ldots$ & $2.86 \mathrm{e}-15$ & $\ldots$ & HLL-55,05413693-0153393,B-21,M-34 \\
\hline 68 & J054137.1-014659 & 54137.16 & -14659.4 & $286 \pm 18$ & 1.06 & 1.98 & $1.0_{-0.3}^{+0.6}$ & $1.8_{-0.8}^{+0.2}$ & $4.35 \mathrm{e}-14$ & 30.54 & $05413714-0146597$ \\
\hline 69 & J054137.3-015313 & 54137.34 & -15313.3 & $115 \pm 12$ & 0.51 & 2.33 & $1.3_{-0.5}^{+0.6}$ & $1.9_{-0.6}^{+0.9}$ & $1.75 \mathrm{e}-14$ & 30.09 & HLL-42,HLP-14,05413733-0153132,B-25,M-35 \\
\hline
\end{tabular}


Table 1-Continued

\begin{tabular}{|c|c|c|c|c|c|c|c|c|c|c|c|}
\hline (1) & $\begin{array}{c}\text { Name } \\
(2)\end{array}$ & $\begin{array}{c}\text { R.A. } \\
\text { (J2000) } \\
(3)\end{array}$ & $\begin{array}{c}\text { Decl. } \\
(\mathrm{J} 2000) \\
(4)\end{array}$ & $\begin{array}{c}\text { Net } \\
\text { Counts } \\
(5)\end{array}$ & $\begin{array}{l}\text { KS } \\
(6)\end{array}$ & $\begin{array}{c}\mathrm{E} \\
(\mathrm{keV}) \\
(7)\end{array}$ & $\begin{array}{c}\mathrm{N}_{\mathrm{H}} \\
(8) \\
\left.\mathrm{cm}^{-2}\right)\end{array}$ & $\begin{array}{c}\mathrm{kT} \\
(\mathrm{keV}) \\
(9)\end{array}$ & $\begin{array}{c}\mathrm{F}_{\mathrm{x}, \mathrm{abs}} \\
\left(\mathrm{erg} / \mathrm{cm}^{2} / \mathrm{s}\right) \\
(10)\end{array}$ & $\begin{array}{c}\log \mathrm{L}_{\mathrm{x}} \\
(\mathrm{erg} / \mathrm{s}) \\
(11)\end{array}$ & $\begin{array}{c}\text { Identification } \\
\text { (12) }\end{array}$ \\
\hline 70 & J054137.3-015244 & 54137.39 & -15244.6 & $19 \pm 6$ & 1.54 & 2.56 & & $\ldots$ & $3.86 \mathrm{e}-15$ & $\ldots$ & HLL-34,05413739-0152447,B-26 \\
\hline 71 & J054137.4-014953 & 54137.43 & -14953.1 & $181 \pm 15$ & 0.73 & 2.47 & $1.5_{-0.6}^{+0.7}$ & $2.5_{-0.8}^{+2.0}$ & $2.94 \mathrm{e}-14$ & 30.14 & HLL-3,HLP-12,05413744-0149532 \\
\hline 72 & J054137.4-014810 & 54137.47 & -14810.5 & $204 \pm 15$ & 1.20 & 2.10 & $0.8_{-0.3}^{+0.4}$ & $2.0_{-0.6}^{+1.7}$ & $6.68 \mathrm{e}-14$ & 30.44 & 05413748-0148108 \\
\hline 73 & J054137.5-015652 & 54137.59 & -15652.9 & $11 \pm 4$ & 0.52 & 4.33 & $\ldots$ & $\ldots$ & $3.61 \mathrm{e}-15$ & $\ldots$ & $\ldots$ \\
\hline 74 & J054137.6-015424 & 54137.62 & -15424.5 & $297 \pm 18$ & 1.10 & 2.34 & $1.2_{-0.2}^{+0.3}$ & $2.5_{-0.6}^{+0.7}$ & $4.58 \mathrm{e}-14$ & 30.33 & HLL-80,05413762-0154246,B-28,M-38 \\
\hline $75^{*}$ & J054137.7-015351 & 54137.73 & -15351.4 & $3084 \pm 57$ & 0.99 & 2.46 & $1.4_{-0.1}^{+0.3}$ & $2.6_{-0.2}^{+0.3}$ & $4.97 \mathrm{e}-13$ & 31.38 & HLL-61,05413774-0153514,B-30,M-43 \\
\hline 76 & J054137.7-015509 & 54137.75 & -15509.9 & $10 \pm 4$ & 1.05 & 2.68 & & $\ldots$ & $1.51 \mathrm{e}-15$ & $\ldots$ & B-31,M-44 \\
\hline 77 & J054137.8-015436 & 54137.85 & -15436.5 & $97 \pm 11$ & 0.60 & 1.51 & $1.1_{-0.4}^{+0.6}$ & $0.5_{-0.2}^{+1.2}$ & $7.54 \mathrm{e}-15$ & 30.30 & HLL-86,HLP-2,05413785-0154364,B89-1,B-33 \\
\hline 78 & J054137.8-015431 & 54137.86 & -15431.8 & $426 \pm 22$ & 0.79 & 2.39 & $1.4_{-0.3}^{+0.3}$ & $2.3_{-0.5}^{+0.8}$ & $6.44 \mathrm{e}-14$ & 30.52 & $05413786-0154323, \mathrm{~V}-2$ \\
\hline 79 & J054137.9-015311 & 54137.90 & -15311.2 & $825 \pm 30$ & 1.47 & 2.18 & $1.1_{-0.1}^{+0.1}$ & $2.1_{-0.3}^{+0.5}$ & $1.03 \mathrm{e}-13$ & 30.68 & B-35,M-48,05413789-0153112 \\
\hline 80 & J054138.0-015357 & 54138.08 & -15357.2 & $238 \pm 16$ & 2.00 & 2.33 & $1.4_{-0.3}^{+0.4}$ & $2.9_{-0.9}^{+2.03}$ & $3.30 \mathrm{e}-14$ & 30.15 & HLL-64,05413807-0153572,B-37,M-51 \\
\hline $81 *_{\mathrm{f}}$ & J054138.1-015817 & 54138.16 & -15817.6 & $433 \pm 22$ & 8.76 & 2.71 & $1.0_{-0.2}^{+0.3}$ & $8.3_{-3.6}^{+\ldots .9}$ & $7.86 \mathrm{e}-14$ & 30.44 & HLL-137,05413816-0158176 \\
\hline 82 & J054138.1-015454 & 54138.20 & -15454.8 & $17 \pm 5$ & 0.70 & 3.06 & $\ldots$ & $\ldots$ & $3.39 \mathrm{e}-15$ & $\ldots$ & HLL-94,05413818-0154549,B-40,M-54 \\
\hline 83 & J054138.2-015536 & 54138.21 & -15536.2 & $67 \pm 9$ & 0.79 & 2.02 & $\ldots$ & $\ldots$ & $2.49 \mathrm{e}-14$ & $\ldots$ & 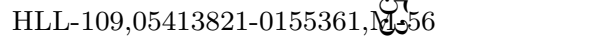 \\
\hline 84 & J054138.2-014633 & 54138.22 & -14633.6 & $42 \pm 8$ & 0.56 & 1.86 & $\ldots$ & $\ldots$ & $7.53 \mathrm{e}-15$ & $\ldots$ & 05413819-0146300 | \\
\hline $85 * f$ & J054138.2-015309 & 54138.24 & -15309.1 & $2496 \pm 51$ & 5.37 & 2.79 & $1.8_{-0.2}^{+0.2}$ & $3.6_{-0.4}^{+0.5}$ & $4.47 \mathrm{e}-13$ & 31.34 & HLL-37,05413824-0153090,B-43,B89-11 \\
\hline 86 & J054138.3-015332 & 54138.32 & -15332.9 & $79 \pm 10$ & 0.68 & 1.05 & $\ldots$ & $\ldots$ & $4.79 \mathrm{e}-15$ & $\ldots$ & 05413832-0153331,B-45,M-60 \\
\hline 87 & J054138.3-015558 & 54138.38 & -15558.2 & $17 \pm 5$ & 0.70 & 2.48 & $\ldots$ & $\ldots$ & $2.69 \mathrm{e}-15$ & $\ldots$ & M-61,05413840-0155582 \\
\hline 88 & J054138.4-015037 & 54138.41 & -15038.0 & $309 \pm 19$ & 0.73 & 2.72 & $1.6_{-0.4}^{+0.7}$ & $3.2_{-1.0}^{+2.1}$ & $5.75 \mathrm{e}-14$ & 30.46 & HLL-10,HLP-33,05413843-0150383 \\
\hline 89 & J054138.5-015322 & 54138.59 & -15322.7 & $1869 \pm 44$ & 1.66 & 2.63 & $1.9_{-0.1}^{+0.2}$ & $2.5_{-0.2}^{+0.0}$ & $3.01 \mathrm{e}-13$ & 31.22 & HLL-45,05413858-0153227,B89-10,B-47 \\
\hline 90 & J054138.5-015730 & 54138.60 & -15730.0 & $61 \pm 9$ & 0.83 & 2.06 & $\ldots$ & $\ldots$ & $7.78 \mathrm{e}-15$ & $\ldots$ & HLL-130,05413861-0157301 \\
\hline 91 & J054138.7-015246 & 54138.72 & -15246.0 & $25 \pm 6$ & 0.69 & 2.58 & $\ldots$ & $\ldots$ & $5.87 \mathrm{e}-15$ & $\ldots$ & $05413871-0152463$ \\
\hline 92 & J054138.7-015429 & 54138.72 & -15429.1 & $57 \pm 9$ & 1.46 & 2.94 & $\ldots$ & $\ldots$ & $1.05 \mathrm{e}-14$ & $\ldots$ & B-48,M-67,05413871-0154289 \\
\hline $93 * \mathrm{f}$ & J054138.7-015602 & 54138.76 & -15602.3 & $385 \pm 21$ & 10.2 & 2.89 & $2.0_{-0.4}^{+0.4}$ & $3.7_{-1.0}^{+1.8}$ & $7.49 \mathrm{e}-14$ & 30.59 & HLL-114,M-68,05413876-0156024 \\
\hline $94^{*}$ & J054138.9-015936 & 54138.95 & -15936.1 & $2108 \pm 47$ & 4.01 & 1.92 & $0.4_{-0.1}^{+0.1}$ & $2.8_{-0.3}^{+0.0}$ & $2.36 \mathrm{e}-13$ & 30.86 & HLP-40,Haro 5-59,05413895-0159362 \\
\hline 95 & J054139.0-015145 & 54139.00 & -15145.3 & $26 \pm 6$ & 0.67 & 2.27 & $\ldots$ & $\ldots$ & $4.25 \mathrm{e}-15$ & $\ldots$ & HLL-21,05413901-0151453 \\
\hline $96 f$ & J054139.0-015626 & 54139.06 & -15626.4 & $214 \pm 16$ & 5.23 & 3.24 & $4.4_{-0.6}^{+1.8}$ & $2.1_{-0.6}^{+1.1}$ & $4.92 \mathrm{e}-14$ & 30.72 & HLL-122,M-71,05413906-0156264 \\
\hline 97 & J054139.0-015358 & 54139.09 & -15358.1 & $153 \pm 13$ & 1.20 & 2.39 & $1.9_{-0.5}^{+0.5}$ & $1.2_{-0.1}^{+0.6}$ & $2.17 \mathrm{e}-14$ & 30.18 & HLL-65,HLP-24,05413908-0153582,B-50,M-74 \\
\hline 98 & J054139.1-015207 & 54139.12 & -15207.3 & $672 \pm 27$ & 1.58 & 2.77 & $2.4_{-0.3}^{+0.4}$ & $2.2_{-0.4}^{+0.4}$ & $1.40 \mathrm{e}-13$ & 30.92 & HLL-23,HLP-4,05413913-0152073 \\
\hline $99 *$ & J054139.1-015414 & 54139.19 & -15414.0 & $334 \pm 19$ & 0.57 & 2.62 & $1.6_{-0.4}^{+0.5}$ & $2.5_{-0.6}^{+0.4}$ & $5.28 \mathrm{e}-14$ & 30.38 & HLL-75,HLP-45,05413919-0154140,B-51,M-75 \\
\hline 100 & J054139.2-015307 & 54139.23 & -15308.0 & $12 \pm 5$ & 0.37 & 2.51 & $\ldots$ & $\ldots$ & $1.95 \mathrm{e}-15$ & $\ldots$ & 05413920-0153075,B-53 \\
\hline 101 & J054139.2-015401 & 54139.24 & -15401.7 & $19 \pm 5$ & 1.40 & 2.95 & $\ldots$ & $\ldots$ & $3.64 \mathrm{e}-15$ & $\ldots$ & $\mathrm{B}-52, \mathrm{M}-76$ \\
\hline 102 & J054139.4-015236 & 54139.40 & -15236.8 & $27 \pm 6$ & 0.59 & 3.33 & $\ldots$ & $\ldots$ & $6.59 \mathrm{e}-15$ & $\ldots$ & 05413939-0152368 \\
\hline 103 & J054139.4-015153 & 54139.41 & -15153.9 & $13 \pm 5$ & 0.39 & 4.21 & $\ldots$ & $\ldots$ & $4.94 \mathrm{e}-15$ & $\ldots$ & $\ldots$ \\
\hline $104 * f$ & J054139.4-014700 & 54139.42 & -14700.0 & $296 \pm 19$ & 8.54 & 3.40 & $3.6_{-0.8}^{+1.1}$ & $4.6_{-1.6}^{+5.7}$ & $7.95 \mathrm{e}-14$ & 30.70 & 05413956-0147020 \\
\hline
\end{tabular}


Table 1-Continued

\begin{tabular}{|c|c|c|c|c|c|c|c|c|c|c|c|}
\hline (1) & $\begin{array}{c}\text { Name } \\
(2)\end{array}$ & $\begin{array}{c}\text { R.A. } \\
\text { (J2000) } \\
(3)\end{array}$ & $\begin{array}{c}\text { Decl. } \\
(\mathrm{J} 2000) \\
(4)\end{array}$ & $\begin{array}{c}\text { Net } \\
\text { Counts } \\
(5)\end{array}$ & $\begin{array}{l}\mathrm{KS} \\
(6)\end{array}$ & $\begin{array}{c}\mathrm{E} \\
(\mathrm{keV}) \\
(7)\end{array}$ & $\begin{array}{c}\mathrm{N}_{\mathrm{H}} \\
\left(10^{22} \mathrm{~cm}^{-2}\right) \\
(8)\end{array}$ & $\begin{array}{c}\mathrm{kT} \\
(\mathrm{keV}) \\
(9)\end{array}$ & $\begin{array}{c}\mathrm{F}_{\mathrm{x}, \mathrm{abs}} \\
\left(\mathrm{erg} / \mathrm{cm}^{2} / \mathrm{s}\right) \\
(10)\end{array}$ & $\begin{array}{c}\log L_{x} \\
(\mathrm{erg} / \mathrm{s}) \\
(11)\end{array}$ & Identification \\
\hline $105^{*}$ & J054139.4-015326 & 54139.49 & -15326.7 & $276 \pm 18$ & 2.31 & 2.89 & $1.6_{-0.4}^{+0.3}$ & $5.6_{-2.0}^{+\ldots}$ & $5.62 \mathrm{e}-14$ & 30.40 & HLL-49;HLP-49,05413948-0153268,B-55,M-78 \\
\hline 106 & J054139.5-015441 & 54139.55 & -15441.6 & $32 \pm 7$ & 0.49 & 2.77 & $\ldots$ & $\ldots$ & $5.69 \mathrm{e}-15$ & $\ldots$ & 05413956-0154416,B-57,M-80 \\
\hline 107 & J054139.7-020224 & 54139.70 & -20224.2 & $380 \pm 21$ & 2.40 & 2.19 & $0.9_{-0.2}^{+0.3}$ & $2.4_{-0.5}^{+0.8}$ & $5.20 \mathrm{e}-14$ & 30.40 & 05413972-0202242 \\
\hline $108 * f$ & J054140.0-015335 & 54140.05 & -15335.6 & $855 \pm 30$ & 6.49 & 2.96 & $2.4_{-0.3}^{+0.3}$ & $3.3_{-0.6}^{+1.0}$ & $1.71 \mathrm{e}-13$ & 30.96 & B-58,M-84 \\
\hline 109 & J054140.1-020059 & 54140.16 & -20059.2 & $15 \pm 5$ & 0.81 & 2.36 & $\ldots$ & $\ldots$ & $3.53 \mathrm{e}-15$ & $\ldots$ & 05414012-0201000 \\
\hline 110 & J054140.1-015407 & 54140.20 & -15407.2 & $258 \pm 17$ & 1.66 & 3.44 & $5.4_{-1.2}^{+2.5}$ & $2.4_{-0.9}^{+1.2}$ & $5.85 \mathrm{e}-14$ & 30.75 & 05414019-0154073,B-60,M-87 \\
\hline 111 & J054140.2-015334 & 54140.20 & -15334.0 & $111 \pm 12$ & 1.78 & 2.87 & $2.7_{-1.6}^{+\ldots .2}$ & $2.6_{-2.0}^{+\ldots .9}$ & $2.21 \mathrm{e}-14$ & 29.76 & HLL-53,HLP-61,05414016-0153344,M-86 \\
\hline 112 & J054140.2-015052 & 54140.24 & -15052.5 & $61 \pm 9$ & 0.65 & 2.30 & $\ldots$ & $\ldots$ & $1.00 \mathrm{e}-14$ & $\ldots$ & HLL-12,05414026-0150522 \\
\hline 113 & J054140.2-015643 & 54140.25 & -15643.8 & $85 \pm 10$ & 3.26 & 3.29 & $3.8_{-1.9}^{+2.9}$ & $3.8_{-2.2}^{+\ldots}$ & $1.95 \mathrm{e}-14$ & 30.14 & $05414025-0156439$ \\
\hline $114^{*}$ & J054140.2-015220 & 54140.26 & -15220.5 & $205 \pm 15$ & 3.18 & 2.85 & $1.3_{-0.3}^{+0.5}$ & $5.3_{-2.2}^{+\ldots .2}$ & $3.95 \mathrm{e}-14$ & 30.27 & HLL-28,05414026-0152205 \\
\hline 115 & J054140.5-015221 & 54140.52 & -15221.1 & $46 \pm 8$ & 1.02 & 2.36 & $\ldots$ & $\ldots$ & $6.65 \mathrm{e}-15$ & $\ldots$ & $05414051-0152212$ \\
\hline 116 & J054140.6-015043 & 54140.64 & -15043.1 & $31 \pm 7$ & 1.46 & 2.43 & $\ldots$ & $\ldots$ & $4.88 \mathrm{e}-15$ & $\ldots$ & 05414067-0150431 \\
\hline 117 & J054140.9-015408 & 54140.91 & -15408.8 & $12 \pm 5$ & 0.78 & 3.72 & $\ldots$ & $\ldots$ & $3.12 \mathrm{e}-15$ & $\ldots$ & 05414091-0154087,M-92 \\
\hline 118 & J054141.0-015158 & 54141.02 & -15158.1 & $45 \pm 8$ & 0.96 & 2.86 & $\ldots$ & $\ldots$ & $8.46 \mathrm{e}-15$ & $\ldots$ & $05414102-0151577$ \\
\hline 119 & J054141.3-015303 & 54141.32 & -15303.3 & $17 \pm 5$ & 1.26 & 3.35 & $\ldots$ & $\ldots$ & $3.61 \mathrm{e}-15$ & $\ldots$ & $05414134-0153033$ \\
\hline $120^{*}$ & J054141.3-015332 & 54141.34 & -15332.6 & $7379 \pm 87$ & 1.41 & 3.67 & $4.4_{-0.2}^{+0.4}$ & $6.0_{-0.8}^{+0.8}$ & $2.11 \mathrm{e}-12$ & 32.13 & HLL-52,05414134-0153326,M-95,V-3 \\
\hline 121 & J054141.3-015437 & 54141.35 & -15437.8 & $16 \pm 5$ & 1.50 & 2.98 & $\ldots$ & $\ldots$ & $2.97 \mathrm{e}-15$ & $\ldots$ & M-96 \\
\hline 122 & J054141.3-015352 & 54141.38 & -15352.0 & $47 \pm 8$ & 0.47 & 2.77 & $\ldots$ & $\ldots$ & $9.45 \mathrm{e}-15$ & $\ldots$ & HLL-60,05414138-0153522,B-66,M-98 \\
\hline $123^{*}$ & J054141.3-015444 & 54141.38 & -15444.5 & $692 \pm 27$ & 3.33 & 3.20 & $4.1_{-0.5}^{+0.6}$ & $2.3_{-0.4}^{+0.5}$ & $1.57 \mathrm{e}-13$ & 31.13 & 05414138-0154445,B-65,M-97,V-4 \\
\hline 124 & J054141.4-015439 & 54141.49 & -15439.2 & $90 \pm 11$ & 1.94 & 3.19 & $4.6_{-1.3}^{+2.8}$ & $1.9_{-0.8}^{+2.0}$ & $2.06 \mathrm{e}-14$ & 30.33 & HLL-87,05414148-0154390,B-67,M-102,V-5 \\
\hline 125 & J054141.6-015754 & 54141.64 & -15754.5 & $94 \pm 11$ & 0.92 & 3.30 & $3.6_{-1.7}^{+1.3}$ & $3.4_{-1.8}^{+\ldots .8}$ & $2.13 \mathrm{e}-14$ & 30.21 & HLL-136,HLP-38,05414164-0157545 \\
\hline 126 & J054141.6-020134 & 54141.66 & -20134.2 & $30 \pm 7$ & 1.12 & 3.44 & $\ldots^{-1.7}$ & $\ldots$ & $1.10 \mathrm{e}-14$ & $\ldots$ & $\ldots$ \\
\hline 127 & J054141.6-015412 & 54141.66 & -15412.7 & $31 \pm 7$ & 0.28 & 2.98 & $\ldots$ & $\ldots$ & $8.50 \mathrm{e}-15$ & $\ldots$ & HLL-73,05414167-0154128,M-107 \\
\hline 128 & J054141.7-015344 & 54141.71 & -15344.3 & $18 \pm 5$ & 0.53 & 3.63 & $\ldots$ & $\ldots$ & $5.54 \mathrm{e}-15$ & $\ldots$ & M-109,05414170-0153444 \\
\hline 129 & J054141.7-015334 & 54141.73 & -15334.8 & $24 \pm 6$ & 0.99 & 3.86 & $\ldots$ & $\ldots$ & $7.55 \mathrm{e}-15$ & $\ldots$ & M-108 \\
\hline 130 & J054141.7-015330 & 54141.73 & -15330.1 & $23 \pm 6$ & 0.71 & 4.25 & $\ldots$ & $\ldots$ & $1.13 \mathrm{e}-14$ & $\ldots$ & $\ldots$ \\
\hline 131 & J054141.7-015145 & 54141.76 & -15145.7 & $109 \pm 11$ & 1.97 & 2.04 & $0.8_{-0.3}^{+0.4}$ & $2.4_{-0.8}^{+1.5}$ & $1.29 \mathrm{e}-14$ & 29.67 & HLL-20,05414177-0151456 \\
\hline 132 & J054141.7-015459 & 54141.79 & -15500.0 & $66 \pm 9$ & 0.69 & 2.88 & $\ldots$ & $\ldots$ & $1.27 \mathrm{e}-14$ & $\ldots$ & 05414179-0154599,M-110 \\
\hline $133 * f$ & J054141.9-015423 & 54141.98 & -15423.9 & $314 \pm 19$ & 7.38 & 3.87 & $6.3_{-1.5}^{+1.3}$ & $3.7_{-1.2}^{+\ldots}$ & $1.17 \mathrm{e}-13$ & 30.93 & HLL-78,HLP-80,05414198-0154240,B-73,M-113 \\
\hline 134 & J054142.0-015301 & 54142.09 & -15301.2 & $18 \pm 5$ & 0.55 & 3.20 & $\ldots$ & $\ldots$ & $3.69 \mathrm{e}-15$ & $\ldots$ & 05414211-0153014 \\
\hline 135 & J054142.1-015509 & 54142.20 & -15509.6 & $7 \pm 4$ & 0.87 & 2.82 & $\ldots$ & $\ldots$ & $2.94 \mathrm{e}-15$ & $\ldots$ & HLL-100,05414220-0155099,M-118 \\
\hline 136 & J054142.3-015315 & 54142.36 & -15315.5 & $56 \pm 9$ & 1.10 & 4.08 & $\ldots$ & $\ldots$ & $1.71 \mathrm{e}-14$ & $\ldots$ & 05414236-0153152,M-120 \\
\hline 137 & J054142.4-015502 & 54142.45 & -15502.6 & $164 \pm 14$ & 1.57 & 2.94 & $2.2_{-0.5}^{+0.6}$ & $3.8_{-1.3}^{+3.2}$ & $5.07 \mathrm{e}-14$ & 30.47 & HLL-97,05414245-0155025,M-121 \\
\hline 138 & J054142.4-014800 & 54142.48 & -14800.9 & $133 \pm 13$ & 1.16 & 1.95 & $0.5_{-0.2}^{+0.5}$ & $1.6_{-0.4}^{+0.6}$ & $1.52 \mathrm{e}-14$ & 29.86 & $05414248-0148013$ \\
\hline $139 * f$ & J054142.4-015409 & 54142.49 & -15409.6 & $403 \pm 21$ & 5.24 & 2.93 & $2.1_{-0.4}^{+0.5}$ & $3.2_{-0.9}^{+1.8}$ & $9.02 \mathrm{e}-14$ & 30.72 & HLL-70,05414249-0154097,B-74,M-122 \\
\hline
\end{tabular}


Table 1-Continued

\begin{tabular}{|c|c|c|c|c|c|c|c|c|c|c|c|}
\hline (1) & $\begin{array}{c}\text { Name } \\
(2)\end{array}$ & $\begin{array}{c}\text { R.A. } \\
\text { (J2000) } \\
(3)\end{array}$ & $\begin{array}{c}\text { Decl. } \\
(\mathrm{J} 2000) \\
(4)\end{array}$ & $\begin{array}{c}\text { Net } \\
\text { Counts } \\
(5)\end{array}$ & $\begin{array}{l}\mathrm{KS} \\
\text { (6) }\end{array}$ & $\begin{array}{c}\mathrm{E} \\
(\mathrm{keV}) \\
(7)\end{array}$ & $\begin{array}{c}\mathrm{N}_{\mathrm{H}} \\
\left(10^{22} \mathrm{~cm}^{-2}\right) \\
(8)\end{array}$ & $\begin{array}{c}\mathrm{kT} \\
(\mathrm{keV}) \\
(9)\end{array}$ & $\begin{array}{c}\mathrm{F}_{\mathrm{x}, \mathrm{abs}} \\
\left(\mathrm{erg} / \mathrm{cm}^{2} / \mathrm{s}\right) \\
(10)\end{array}$ & $\begin{array}{c}\log \mathrm{L}_{\mathrm{x}} \\
(\mathrm{erg} / \mathrm{s}) \\
(11)\end{array}$ & $\begin{array}{l}\text { Identification } \\
\qquad(12)\end{array}$ \\
\hline 140 & J054142.5-015617 & 54142.54 & -15617.6 & $26 \pm 6$ & 1.25 & 1.67 & $\ldots$ & $\ldots$ & $1.97 \mathrm{e}-15$ & $\ldots$ & HLL-117,05414254-0156175,M-124 \\
\hline $141 *_{\mathrm{f}}$ & J054142.6-015445 & 54142.62 & -15445.6 & $513 \pm 24$ & 2.57 & 2.90 & $2.6_{-0.5}^{+0.5}$ & $3.4_{-0.8}^{+2.8}$ & $1.16 \mathrm{e}-13$ & 30.80 & HLL-90,05414261-0154456,B-76,M-128 \\
\hline 142 & J054142.8-015436 & 54142.81 & -15436.0 & $686 \pm 27$ & 1.49 & 2.75 & $2.3_{-0.4}^{+0.3}$ & $2.3_{-0.3}^{+0.5}$ & $1.35 \mathrm{e}-13$ & 30.90 & HLL-85,05414280-0154360,B-78,M-133 \\
\hline 143 & J054142.8-015317 & 54142.85 & -15317.0 & $13 \pm 5$ & 0.77 & 3.34 & $\ldots$ & $\ldots$ & $2.76 \mathrm{e}-15$ & $\ldots$ & 05414288-0153163,M-135 \\
\hline 144 & J054143.0-015440 & 54143.03 & -15440.2 & $23 \pm 6$ & 1.18 & 3.57 & $\ldots$ & $\ldots$ & $6.48 \mathrm{e}-15$ & $\ldots$ & M-136 \\
\hline $145^{*}$ & J054143.1-020354 & 54143.12 & -20354.0 & $462 \pm 23$ & 2.93 & 1.30 & $0.2_{-0.1}^{+0.2}$ & $\ldots$ & $4.28 \mathrm{e}-14$ & $\ldots$ & 05414316-0203541 \\
\hline 146 & J054143.2-015331 & 54143.29 & -15331.5 & $30 \pm 7$ & 3.96 & 2.83 & $\ldots$ & $\ldots$ & $5.40 \mathrm{e}-15$ & $\ldots$ & HLL-51 \\
\hline 147 & J054143.4-015642 & 54143.44 & -15642.4 & $285 \pm 18$ & 1.05 & 2.65 & $2.2_{-0.4}^{+0.5}$ & $2.3_{-0.6}^{+0.7}$ & $4.78 \mathrm{e}-14$ & 30.48 & HLL-124,B89-24,05414344-0156425,V-6 \\
\hline $148^{*}$ & J054143.4-020349 & 54143.45 & -20349.0 & $464 \pm 23$ & 2.93 & 1.30 & $0.2_{-0.2}^{+0.2}$ & $\ldots$ & $4.37 \mathrm{e}-14$ & $\ldots$ & $05414344-0203482$ \\
\hline 149 & J054143.4-015326 & 54143.49 & -15326.3 & $20 \pm 6$ & 1.68 & 4.47 & $\ldots$ & $\ldots$ & $6.87 \mathrm{e}-15$ & $\ldots$ & M96-146 \\
\hline 150 & J054143.4-014837 & 54143.50 & -14837.1 & $16 \pm 6$ & 0.94 & 3.63 & $\ldots$ & $\ldots$ & $4.35 \mathrm{e}-15$ & $\ldots$ & $\ldots$ \\
\hline 151 & J054143.5-015511 & 54143.55 & -15511.7 & $24 \pm 6$ & 2.25 & 4.48 & $\ldots$ & $\ldots$ & $2.02 \mathrm{e}-14$ & $\ldots$ & 05414362-0155114,M-147 \\
\hline $152^{*}$ & J054143.5-015356 & 54143.55 & -15356.6 & $140 \pm 13$ & 0.87 & 3.03 & $6.1_{-0.9}^{+1.2}$ & $1.4_{-0.3}^{+0.2}$ & $2.62 \mathrm{e}-14$ & 30.83 & 05414356-0153567,M-149,V-7 \\
\hline $153^{*}$ & J054143.7-014645 & 54143.79 & -14645.4 & $743 \pm 29$ & 1.62 & 1.56 & $0.3_{-0.1}^{+0.9}$ & $\ldots$ & $7.13 \mathrm{e}-14$ & 30.41 & 05414380-0146457 ल \\
\hline 154 & J054143.8-015621 & 54143.82 & -15622.0 & $258 \pm 17$ & 2.62 & 3.57 & $5.2_{-1.3}^{+0.1}$ & $3.1_{-1.0}^{+2.2}$ & $6.36 \mathrm{e}-14$ & 30.69 & 05414383-0156220,M-153,B89-31 \\
\hline 155 & J054143.8-015338 & 54143.85 & -15338.3 & $19 \pm 5$ & 0.88 & 3.03 & $\ldots$ & $\ldots$ & $3.43 \mathrm{e}-15$ & $\ldots$ & 05414384-0153383,M-154 \\
\hline 156 & J054143.9-015329 & 54143.91 & -15329.1 & $187 \pm 15$ & 1.42 & 3.29 & $2.7_{-0.7}^{+0.8}$ & $4.2_{-1.5}^{+6.0}$ & $4.16 \mathrm{e}-14$ & 30.39 & 05414391-0153291,M-156 \\
\hline 157 & J054143.9-015554 & 54143.94 & -15554.8 & $10 \pm 4$ & 0.87 & 3.65 & $\ldots$ & $\ldots$ & $2.17 \mathrm{e}-15$ & $\ldots$ & $\ldots$ \\
\hline 158 & J054143.9-015845 & 54143.96 & -15845.1 & $22 \pm 6$ & 2.01 & 3.04 & $\ldots$ & $\ldots$ & $4.12 \mathrm{e}-15$ & $\ldots$ & HLL-139,05414398-0158454 \\
\hline 159 & J054143.9-015302 & 54143.99 & -15302.2 & $9 \pm 4$ & 0.62 & 3.25 & $\cdots$ & $\cdots$ & $1.95 \mathrm{e}-15$ & $\cdots$ & 05414397-0153019 \\
\hline $160 * \mathrm{f}$ & J054144.1-015347 & 54144.12 & -15347.1 & $301 \pm 18$ & 6.24 & 4.16 & $15.1_{-4.8}^{+5.1}$ & $2.0_{-0.6}^{+1.3}$ & $8.54 \mathrm{e}-14$ & 31.38 & 05414412-0153472,M-165 \\
\hline 161 & J054144.1-015520 & 54144.17 & -15520.4 & $34 \pm 7$ & 0.85 & 3.82 & $\ldots$ & $\ldots$ & $9.45 \mathrm{e}-15$ & $\ldots$ & M-167 \\
\hline 162 & J054144.1-015735 & 54144.18 & -15735.5 & $8 \pm 4$ & 0.54 & 1.87 & $\ldots$ & $\ldots$ & $6.53 \mathrm{e}-16$ & $\ldots$ & $05414420-0157353$ \\
\hline 163 & J054144.2-015404 & 54144.22 & -15404.9 & $48 \pm 8$ & 1.07 & 3.54 & $\ldots$ & $\ldots$ & $1.14 \mathrm{e}-14$ & $\ldots$ & 05414422-0154051,M-170 \\
\hline 164 & J054144.3-015524 & 54144.33 & -15524.8 & $34 \pm 7$ & 0.58 & 2.27 & $\ldots$ & $\ldots$ & $4.21 \mathrm{e}-15$ & $\ldots$ & $\mathrm{M}-173$ \\
\hline 165 & J054144.3-015420 & 54144.36 & -15420.2 & $74 \pm 10$ & 1.48 & 2.95 & $\ldots$ & $\ldots$ & $1.34 \mathrm{e}-14$ & $\ldots$ & 05414436-0154203,B-84,M-174 \\
\hline 166 & J054144.3-015335 & 54144.37 & -15335.8 & $97 \pm 11$ & 1.32 & 3.44 & $5.0_{-1.7}^{+2.9}$ & $2.3_{-1.0}^{+3.4}$ & $2.19 \mathrm{e}-14$ & 30.30 & 05414436-0153358,M-176 \\
\hline 167 & J054144.4-015522A & 54144.40 & -15522.8 & $138 \pm 13$ & 0.85 & 2.44 & $2.1_{-0.3}^{+0.5}$ & $2.5_{-0.6}^{+0.0}$ & $2.07 \mathrm{e}-14$ & 30.11 & HLL-107,HLP-58,05414440-0155229,B89-3 \\
\hline 168 & J054144.4-015522B & 54144.49 & -15522.0 & $56 \pm 9$ & 0.60 & 3.28 & $\ldots$ & $\ldots$ & $1.28 \mathrm{e}-14$ & $\ldots$ & $\ldots$ \\
\hline 169 & J054144.5-015740 & 54144.50 & -15740.2 & $266 \pm 17$ & 1.13 & 1.99 & $0.8_{-0.2}^{+0.2}$ & $2.1_{-0.4}^{+0.4}$ & $3.09 \mathrm{e}-14$ & 30.09 & HLL-133,05414451-0157402,B89-29 \\
\hline 170 & J054144.7-015348 & 54144.74 & -15348.5 & $39 \pm 7$ & 1.83 & 3.91 & $\ldots$ & $\ldots$ & $1.06 \mathrm{e}-14$ & $\ldots$ & M-182,05414474-0153485 \\
\hline 171 & J054144.8-015425 & 54144.82 & -15425.1 & $47 \pm 8$ & 1.04 & 3.11 & $\ldots$ & $\ldots$ & $8.77 \mathrm{e}-15$ & $\ldots$ & HLL-77,HLP-22,05414482-0154251,B-85,V-11 \\
\hline 172 & J054144.8-015435 & 54144.84 & -15435.9 & $15 \pm 5$ & 0.79 & 3.29 & $\ldots$ & $\ldots$ & $3.08 \mathrm{e}-15$ & $\ldots$ & 05414483-0154357,B-86,M-184,HLP-73 \\
\hline 173 & J054144.9-015717 & 54144.96 & -15717.3 & $41 \pm 7$ & 0.55 & 0.96 & $\ldots$ & $\ldots$ & $2.59 \mathrm{e}-15$ & $\ldots$ & 05414496-0157174 \\
\hline $174^{*} \mathrm{f}$ & J054145.0-015406 & 54145.06 & -15406.2 & $651 \pm 27$ & 16.0 & 3.72 & $4.2_{-0.5}^{+0.7}$ & $6.1_{-2.1}^{+3.1}$ & $1.70 \mathrm{e}-13$ & 31.01 & HLP-74,05414506-0154063,M-188 \\
\hline
\end{tabular}


Table 1-Continued

\begin{tabular}{|c|c|c|c|c|c|c|c|c|c|c|c|}
\hline (1) & $\begin{array}{l}\text { Name } \\
(2)\end{array}$ & $\begin{array}{c}\text { R.A. } \\
(\mathrm{J} 2000) \\
(3)\end{array}$ & $\begin{array}{c}\text { Decl. } \\
(\mathrm{J} 2000) \\
(4)\end{array}$ & $\begin{array}{c}\text { Net } \\
\text { Counts } \\
(5)\end{array}$ & $\begin{array}{l}\text { KS } \\
(6)\end{array}$ & $\begin{array}{c}\mathrm{E} \\
(\mathrm{keV}) \\
(7)\end{array}$ & $\begin{array}{c}\mathrm{N}_{\mathrm{H}} \\
\left(10^{22} \mathrm{~cm}^{-2}\right) \\
(8)\end{array}$ & $\begin{array}{c}\mathrm{kT} \\
(\mathrm{keV}) \\
(9)\end{array}$ & $\begin{array}{c}\mathrm{F}_{\mathrm{x}, \mathrm{abs}} \\
\left(\mathrm{erg} / \mathrm{cm}^{2} / \mathrm{s}\right) \\
(10)\end{array}$ & $\begin{array}{c}\log L_{x} \\
(\mathrm{erg} / \mathrm{s}) \\
(11)\end{array}$ & $\begin{array}{l}\text { Identification } \\
\text { (12) }\end{array}$ \\
\hline $175 * \mathrm{f}$ & J054145.0-015144 & 54145.08 & -15144.3 & $1810 \pm 44$ & 10.1 & 3.03 & $2.3_{-0.2}^{+0.2}$ & $3.7_{-0.5}^{+0.7}$ & $3.70 \mathrm{e}-13$ & 31.26 & HLL-19,05414508-0151443 \\
\hline 176 & J054145.2-015422 & 54145.23 & -15422.7 & $196 \pm 15$ & 1.40 & 3.03 & $3.9_{-0.9}^{+1.2}$ & $1.9_{-0.5}^{+0.8}$ & $3.75 \mathrm{e}-14$ & 30.59 & B-89,M-190 \\
\hline 177 & J054145.3-015502 & 54145.33 & -15502.6 & $16 \pm 5$ & 2.11 & 3.00 & $\ldots$ & $\ldots$ & $5.90 \mathrm{e}-15$ & $\ldots$ & 05414532-0155025,M-193 \\
\hline 178 & J054145.3-015156 & 54145.38 & -15156.6 & $224 \pm 16$ & 0.49 & 3.22 & $3.4_{-0.8}^{+1.1}$ & $2.9_{-0.9}^{+1.8}$ & $4.98 \mathrm{e}-14$ & 30.52 & 05414538-0151566 \\
\hline 179 & J054145.3-015355 & 54145.40 & -15355.7 & $13 \pm 5$ & 0.48 & 2.58 & $\ldots$ & $\ldots$ & $2.17 \mathrm{e}-15$ & $\ldots$ & M-195 \\
\hline 180 & J054145.4-015425 & 54145.42 & -15425.7 & $64 \pm 9$ & 0.76 & 3.97 & $\ldots$ & $\ldots$ & $1.74 \mathrm{e}-14$ & $\ldots$ & $\ldots$ \\
\hline 181 & J054145.4-015616 & 54145.48 & -15617.0 & $8 \pm 4$ & 0.19 & 0.92 & $\ldots$ & $\ldots$ & $3.96 \mathrm{e}-16$ & $\ldots$ & HLL-116,05414548-0156169,M-196,B89-22 \\
\hline $182^{*}$ & J054145.4-015428 & 54145.48 & -15428.6 & $371 \pm 20$ & 1.41 & 3.14 & $3.3_{-0.5}^{+0.9}$ & $2.6_{-0.6}^{+0.7}$ & $7.42 \mathrm{e}-14$ & 30.65 & 05414550-0154286,B-90,M-197,IRS2b,V-15 \\
\hline $183 * \mathrm{f}$ & J054145.5-015426 & 54145.52 & -15426.9 & $220 \pm 16$ & 5.94 & 3.41 & $2.9_{-1.0}^{+0.0}$ & $6.7_{-3.1}^{+\ldots .6}$ & $5.28 \mathrm{e}-14$ & 30.39 & $\mathrm{~V}-16$ \\
\hline 184 & J054145.6-015732 & 54145.61 & -15733.0 & $34 \pm 7$ & 0.95 & 1.60 & $\ldots$ & $\ldots$ & $3.47 \mathrm{e}-15$ & $\ldots$ & $05414562-0157330$ \\
\hline 185 & J054145.6-015504 & 54145.69 & -15504.6 & $14 \pm 5$ & 0.75 & 3.98 & $\ldots$ & $\ldots$ & $6.56 \mathrm{e}-15$ & $\ldots$ & M-200 \\
\hline 186 & J054145.7-015212 & 54145.72 & -15212.8 & $10 \pm 4$ & 0.57 & 1.91 & $\ldots$ & $\ldots$ & $8.49 \mathrm{e}-16$ & $\ldots$ & $05414576-0152130$ \\
\hline $187^{*}$ & J054145.7-015429 & 54145.80 & -15429.8 & $162 \pm 14$ & 1.30 & 3.42 & $3.1_{-0.8}^{+1.0}$ & $4.9_{-2.1}^{+\ldots}$ & $3.74 \mathrm{e}-14$ & 30.37 & HLP-1,B89-2,0541458-0154297,B-92,M-205,V-19 \\
\hline $188^{*} \mathrm{f}$ & J054145.8-015410 & 54145.89 & -15410.9 & $1065 \pm 34$ & 2.53 & 3.31 & $3.9_{-0.3}^{+0.8}$ & $2.8_{-0.4}^{+0.5}$ & $2.47 \mathrm{e}-13$ & 31.25 & $\mathrm{M}-206, \mathrm{~V}-20$ नु \\
\hline $189^{*}$ & J054145.9-015626 & 54145.94 & -15626.4 & $176 \pm 14$ & 1.45 & 3.88 & $5.5_{-1.9}^{+0.6}$ & $\ldots$ & $5.03 \mathrm{e}-14$ & 30.54 & 05414594-0156263,M-207,B89+23 \\
\hline 190 & J054145.9-015501 & 54145.97 & -15501.9 & $65 \pm 9$ & 1.61 & 3.41 & $\ldots$ & $\ldots$ & $2.42 \mathrm{e}-14$ & $\ldots$ & HLL-96,HLP-92,M-208 \\
\hline 191 & J054146.0-015653 & 54146.02 & -15653.8 & $33 \pm 7$ & 1.38 & 3.76 & $\ldots$ & $\ldots$ & $8.40 \mathrm{e}-15$ & $\ldots$ & $05414602-0156538$ \\
\hline $192 * \mathrm{f}$ & J054146.1-015414 & 54146.11 & -15414.7 & $1753 \pm 43$ & 27.4 & 3.89 & $3.7_{-0.5}^{+0.5}$ & $\ldots$ & $5.20 \mathrm{e}-13$ & var. & 05414611-0154147,B-93,M-210 \\
\hline $193^{*}$ & J054146.1-015621 & 54146.15 & -15621.9 & $397 \pm 21$ & 2.96 & 4.24 & $9.6_{-1.8}^{+2.4}$ & $5.0_{-1.9}^{+5.4}$ & $1.27 \mathrm{e}-13$ & 31.07 & $\mathrm{~V}-21$ \\
\hline 194 & J054146.2-015346 & 54146.20 & -15346.7 & $66 \pm 9$ & 0.79 & 2.49 & $\ldots^{-1.8}$ & $\ldots^{-1.9}$ & $2.00 \mathrm{e}-14$ & $\ldots$ & HLL-58,05414621-0153466,M-212,B89-7 \\
\hline 195 & J054146.2-015533 & 54146.23 & -15533.1 & $165 \pm 14$ & 1.89 & 4.15 & $11.8_{-5.3}^{+10.0}$ & $3.3_{-1.9}^{+\ldots .}$ & $4.84 \mathrm{e}-14$ & 30.86 & M-211 \\
\hline $196^{*}$ & J054146.2-015654 & 54146.23 & -15654.6 & $150 \pm 13$ & 1.17 & 4.07 & $5.2_{-1.4}^{+2.5 .3}$ & $\ldots^{-1.9}$ & $4.50 \mathrm{e}-14$ & 30.41 & $\mathrm{~V}-22$ \\
\hline 197 & J054146.2-015554 & 54146.26 & -15554.1 & $32 \pm 7$ & 0.34 & 4.77 & $\ldots$ & $\ldots$ & $1.18 \mathrm{e}-14$ & $\ldots$ & $\ldots$ \\
\hline 198 & J054146.4-015011 & 54146.40 & -15011.3 & $47 \pm 8$ & 0.91 & 2.01 & $\ldots$ & $\ldots$ & $5.64 \mathrm{e}-15$ & $\ldots$ & HLL-5,05414641-0150111 \\
\hline $199 f$ & J054146.5-015148 & 54146.52 & -15148.6 & $91 \pm 11$ & 6.37 & 3.42 & $3.0_{-0.5}^{+1.3}$ & $4.6_{-2.1}^{+\ldots}$ & $2.33 \mathrm{e}-14$ & 30.17 & $05414652-0151487$ \\
\hline 200 & J054146.5-015446 & 54146.57 & -15446.6 & $94 \pm 11$ & 1.24 & 3.27 & $7.0_{-2.2}^{+0.7}$ & $1.8_{-1.1}^{+1.1 .1}$ & $6.68 \mathrm{e}-14$ & 31.06 & 05414655-0154469,B-94,M-214,V-23 \\
\hline 201 & J054146.8-015447 & 54146.86 & -15447.4 & $16 \pm 5$ & 1.26 & 4.14 & $\ldots$ & $\ldots$ & $2.70 \mathrm{e}-14$ & $\ldots$ & $\mathrm{M}-217$ \\
\hline 202 & J054146.8-014957 & 54146.89 & -14957.1 & $370 \pm 20$ & 0.88 & 2.07 & $0.8_{-0.2}^{+0.2}$ & $2.5_{-0.6}^{+0.7}$ & $4.48 \mathrm{e}-14$ & 30.25 & HLL-4,HLP-23,05414690-0149573 \\
\hline 203 & J054147.0-014926 & 54147.01 & -14926.6 & $449 \pm 22$ & 3.14 & 2.47 & $1.5_{-0.4}^{+0.3}$ & $2.9_{-0.7}^{+1.6}$ & $7.13 \mathrm{e}-14$ & 30.55 & HLL-1,05414702-0149265 \\
\hline 204 & J054147.0-015035 & 54147.10 & -15035.4 & $158 \pm 14$ & 0.51 & 1.93 & $1.0_{-0.4}^{+0.4}$ & $1.3_{-0.3}^{+0.4}$ & $1.67 \mathrm{e}-14$ & 29.99 & HLL-8,HLP-90,05414712-0150353 \\
\hline 205 & J054147.1-020018 & 54147.14 & -20018.8 & $29 \pm 7$ & 0.96 & 1.65 & $\ldots$ & $\ldots$ & $2.27 \mathrm{e}-15$ & $\ldots$ & 05414714-0200190 \\
\hline 206 & J054147.2-015819 & 54147.21 & -15819.7 & $12 \pm 5$ & 0.94 & 2.81 & $\ldots$ & $\ldots$ & $1.98 \mathrm{e}-15$ & $\ldots$ & HLL-138,05414723-0158202 \\
\hline $207^{*} \mathrm{f}$ & J054147.4-015526 & 54147.45 & -15526.3 & $899 \pm 31$ & 17.9 & 3.74 & $4.3_{-0.6}^{+0.7}$ & $7.8_{-2.8}^{+\ldots}$ & $2.40 \mathrm{e}-13$ & 31.11 & $\mathrm{M}-220$ \\
\hline 208 & J054147.9-015541 & 54147.93 & -15541.3 & $24 \pm 6$ & 0.55 & 4.06 & $\ldots$ & $\ldots$ & $6.80 \mathrm{e}-15$ & $\ldots$ & 05414793-0155416,M-225 \\
\hline 209 & J054147.9-015358 & 54147.97 & -15358.4 & $17 \pm 5$ & 0.67 & 3.45 & $\ldots$ & $\ldots$ & $4.13 \mathrm{e}-15$ & $\ldots$ & 05414796-0153585,M-226 \\
\hline
\end{tabular}


Table 1-Continued

\begin{tabular}{|c|c|c|c|c|c|c|c|c|c|c|c|}
\hline No. & $\begin{array}{c}\text { Name } \\
(2)\end{array}$ & $\begin{array}{c}\text { R.A. } \\
\text { (J2000) } \\
(3)\end{array}$ & $\begin{array}{c}\text { Decl. } \\
(\mathrm{J} 2000) \\
(4)\end{array}$ & $\begin{array}{c}\text { Net } \\
\text { Counts } \\
(5)\end{array}$ & $\begin{array}{l}\mathrm{KS} \\
(6)\end{array}$ & $\begin{array}{c}\mathrm{E} \\
(\mathrm{keV}) \\
(7)\end{array}$ & $\begin{array}{c}\mathrm{N}_{\mathrm{H}} \\
\left(10^{22} \mathrm{~cm}^{-2}\right) \\
(8)\end{array}$ & $\begin{array}{c}\mathrm{kT} \\
(\mathrm{keV}) \\
(9)\end{array}$ & $\begin{array}{c}\mathrm{F}_{\mathrm{x}, \mathrm{abs}} \\
\left(\mathrm{erg} / \mathrm{cm}^{2} / \mathrm{s}\right) \\
(10)\end{array}$ & $\begin{array}{c}\log \mathrm{L}_{\mathrm{x}} \\
(\mathrm{erg} / \mathrm{s}) \\
(11)\end{array}$ & $\begin{array}{c}\text { Identification } \\
\text { (12) }\end{array}$ \\
\hline $210 * f$ & J054148.2-015601 & 54148.21 & -15601.8 & $1616 \pm 41$ & 15.4 & 3.61 & $4.5_{-0.3}^{+0.5}$ & $4.6_{-0.9}^{+1.3}$ & $4.17 \mathrm{e}-13$ & 31.45 & 05414821-0156020,M-230,V-24 \\
\hline 211 & J054148.6-015349 & 54148.60 & -15349.5 & $95 \pm 11$ & 0.96 & 2.52 & $1.3_{-0.9}^{+0.7}$ & $3.0_{-1.3}^{+. .9}$ & $1.50 \mathrm{e}-14$ & 29.80 & HLL-59,05414861-0153495 \\
\hline 212 & J054148.6-015540 & 54148.63 & -15541.0 & $9 \pm 4$ & 0.99 & 4.65 & $\ldots$ & $\ldots$ & $3.49 \mathrm{e}-15$ & $\ldots$ & $\ldots$ \\
\hline 213 & J054148.6-015415 & 54148.69 & -15415.6 & $32 \pm 7$ & 2.42 & 2.59 & $\ldots$ & $\ldots$ & $5.46 \mathrm{e}-15$ & $\ldots$ & HLL-74,05414868-0154159 \\
\hline 214 & J054149.3-015332 & 54149.31 & -15332.7 & $76 \pm 10$ & 1.94 & 2.66 & $1.4_{-1.0}^{+0.7}$ & $3.1_{-1.6}^{+\ldots}$ & $1.25 \mathrm{e}-14$ & 29.79 & HLL-50,05414934-0153318 \\
\hline 215 & J054149.6-014601 & 54149.63 & -14601.7 & $146 \pm 13$ & 2.34 & 2.03 & $1.1_{-0.4}^{+0.5}$ & $1.6_{-0.5}^{+0.6}$ & $1.70 \mathrm{e}-14$ & 30.05 & $05414961-0146015$ \\
\hline 216 & J054149.6-015326 & 54149.66 & -15326.9 & $89 \pm 10$ & 0.99 & 2.79 & $1.6_{-0.6}^{+0.7}$ & $4.8_{-2.3}^{+\ldots .5}$ & $1.53 \mathrm{e}-14$ & 29.78 & HLL-48,05414966-0153271 \\
\hline 217 & J054149.8-015255 & 54149.81 & -15255.8 & $21 \pm 6$ & 0.98 & 4.37 & $\ldots$ & $\ldots$ & $6.63 \mathrm{e}-15$ & $\ldots$ & HLL-36,05414983-0152560 \\
\hline 218 & J054149.8-015947 & 54149.88 & -15947.4 & $11 \pm 5$ & 0.47 & 3.39 & $\ldots$ & $\ldots$ & $2.76 \mathrm{e}-15$ & $\ldots$ & 05414984-0159475 \\
\hline 219 & J054150.0-015807 & 54150.04 & -15807.8 & $11 \pm 4$ & 1.29 & 1.56 & $\ldots$ & $\ldots$ & $9.11 \mathrm{e}-16$ & $\ldots$ & 05415006-0158083 \\
\hline 220 & J054150.1-015744 & 54150.15 & -15744.7 & $154 \pm 13$ & 0.95 & 3.12 & $2.5_{-1.2}^{+1.0}$ & $4.2_{-1.8}^{+1.8}$ & $3.26 \mathrm{e}-14$ & 30.18 & HLL-135,HLP-98,05415014-0157449 \\
\hline 221 & J054150.5-015315 & 54150.50 & -15315.2 & $558 \pm 25$ & 1.25 & 2.11 & $0.9_{-0.1}^{+1.22}$ & $2.2_{-0.3}^{+0.8}$ & $6.78 \mathrm{e}-14$ & 30.48 & HLL-41,05415050-0153153,B89-6 \\
\hline $222 *_{\mathrm{f}}$ & J054150.5-020256 & 54150.50 & -20256.9 & $242 \pm 17$ & 6.10 & 3.46 & $4.5_{-0.9}^{+0.13}$ & $3.2_{-1.1}^{+2.5}$ & $6.34 \mathrm{e}-14$ & 30.67 & 05415053-0202570 \\
\hline $223^{*}$ & J054150.6-015306 & 54150.69 & -15306.2 & $22 \pm 6$ & 0.68 & 1.86 & $\ldots$ & $\ldots$ & $2.05 \mathrm{e}-15$ & $\ldots$ & see notes \\
\hline 224 & J054150.7-015548 & 54150.74 & -15548.3 & $13 \pm 5$ & 0.75 & 2.76 & $\ldots$ & $\ldots$ & $2.32 \mathrm{e}-15$ & $\ldots$ & $05415074-0155486$ \\
\hline 225 & J054150.8-015312 & 54150.86 & -15312.7 & $22 \pm 6$ & 0.77 & 2.67 & $\ldots$ & $\ldots$ & $3.40 \mathrm{e}-15$ & $\ldots$ & HLL-40,05415087-0153128 \\
\hline 226 & J054150.8-015722 & 54150.87 & -15722.2 & $7 \pm 4$ & 0.62 & 4.03 & $\ldots$ & $\ldots$ & $2.63 \mathrm{e}-15$ & $\ldots$ & $\ldots$ \\
\hline 227 & J054150.9-014801 & 54150.95 & -14801.6 & $6 \pm 4$ & 0.74 & 3.63 & $\ldots$ & $\ldots$ & $2.59 \mathrm{e}-15$ & $\ldots$ & $\ldots$ \\
\hline 228 & J054150.9-015520 & 54151.00 & -15521.0 & $13 \pm 5$ & 0.39 & 1.10 & $\ldots$ & $\ldots$ & $6.24 \mathrm{e}-16$ & $\ldots$ & $05415100-0155213$ \\
\hline 229 & J054151.6-015216 & 54151.60 & -15216.6 & $66 \pm 9$ & 0.50 & 2.34 & $\ldots$ & $\ldots$ & $1.00 \mathrm{e}-14$ & $\ldots$ & 05415161-0152167 \\
\hline 230 & J054152.0-015518 & 54152.08 & -15518.1 & $18 \pm 5$ & 1.16 & 1.58 & $\ldots$ & $\ldots$ & $1.58 \mathrm{e}-15$ & $\ldots$ & $05415209-0155183$ \\
\hline 231 & J054152.1-015717 & 54152.15 & -15717.3 & $30 \pm 7$ & 1.37 & 2.34 & $\ldots$ & $\ldots$ & $1.35 \mathrm{e}-14$ & $\ldots$ & HLL-127,HLP-75,B89-26,05415215-0157174 \\
\hline $232^{*}$ & J054152.1-020121 & 54152.20 & -20121.1 & $171 \pm 14$ & 0.77 & 2.21 & $1.5_{-0.5}^{+0.6}$ & $1.6_{-0.5}^{+0.6}$ & $2.41 \mathrm{e}-14$ & 30.17 & 05415220-0201211 \\
\hline 233 & J054152.9-015629 & 54152.93 & -15629.3 & $55 \pm 8$ & 0.53 & 3.35 & $\ldots$ & $\ldots$ & $1.42 \mathrm{e}-14$ & $\ldots$ & HLL-121,B89-25,05415292-0156297 \\
\hline $234 *_{\mathrm{f}}$ & J054152.9-015634 & 54152.94 & -15634.8 & $783 \pm 29$ & 6.04 & 2.58 & $1.5_{-0.2}^{+0.3}$ & $3.1_{-0.7}^{+1.1}$ & $1.31 \mathrm{e}-13$ & 30.80 & HLL-123,05415294-0156351 \\
\hline 235 & J054153.1-015743 & 54153.19 & -15743.8 & $59 \pm 9$ & 1.91 & 3.57 & $\ldots{ }^{-0.2}$ & $\ldots$ & $2.96 \mathrm{e}-14$ & $\ldots$ & HLL-134,HLP-78,05415319-0157441,B89-27 \\
\hline 236 & J054153.8-015516 & 54153.87 & -15516.1 & $304 \pm 18$ & 1.04 & 2.77 & $2.5_{-0.5}^{+0.7}$ & $2.3_{-0.7}^{+0.8}$ & $5.19 \mathrm{e}-14$ & 30.55 & HLL-101,HLP-88,05415387-0155163,B89-21 \\
\hline 237 & J054154.1-015817 & 54154.12 & -15817.1 & $12 \pm 5$ & 0.59 & 1.50 & $\ldots$ & $\ldots^{-0.8}$ & $1.57 \mathrm{e}-15$ & $\ldots$ & 05415406-0158170 \\
\hline 238 & J054154.1-014801 & 54154.19 & -14801.7 & $77 \pm 10$ & 1.26 & 1.93 & $\ldots$ & $\ldots$ & $1.02 \mathrm{e}-14$ & $\ldots$ & 05415419-0148017 \\
\hline 239 & J054154.3-015556 & 54154.31 & -15556.2 & $25 \pm 6$ & 1.01 & 1.22 & $\ldots$ & $\ldots$ & $1.67 \mathrm{e}-15$ & $\ldots$ & HLL-112,05415430-0155566 \\
\hline 240 & J054154.7-015726 & 54154.70 & -15727.0 & $33 \pm 7$ & 0.49 & 1.61 & $\ldots$ & $\ldots$ & $2.74 \mathrm{e}-15$ & $\ldots$ & HLL-128,05415472-0157273 \\
\hline 241 & J054154.9-015647 & 54154.93 & -15647.9 & $22 \pm 6$ & 0.56 & 2.24 & $\ldots$ & $\ldots$ & $3.16 \mathrm{e}-15$ & $\ldots$ & HLL-125,05415492-0156483 \\
\hline 242 & J054154.9-015630 & 54154.96 & -15630.9 & $18 \pm 5$ & 1.20 & 3.22 & $\ldots$ & $\ldots$ & $3.82 \mathrm{e}-15$ & $\ldots$ & 05415497-0156312 \\
\hline 243 & J054154.9-015957 & 54154.99 & -15957.8 & $19 \pm 6$ & 0.67 & 3.72 & $\ldots$ & $\ldots$ & $5.00 \mathrm{e}-15$ & $\ldots$ & $\ldots$ \\
\hline 244 & J054155.0-020030 & 54155.04 & -20030.9 & $111 \pm 12$ & 3.36 & 3.13 & $4.2_{-0.5}^{+1.6}$ & $2.5_{-0.9}^{+1.4}$ & $2.34 \mathrm{e}-14$ & 30.30 & 05415505-0200309 \\
\hline
\end{tabular}


Table 1-Continued

\begin{tabular}{|c|c|c|c|c|c|c|c|c|c|c|c|}
\hline (1) & $\begin{array}{c}\text { Name } \\
(2)\end{array}$ & $\begin{array}{c}\text { R.A. } \\
\text { (J2000) } \\
(3)\end{array}$ & $\begin{array}{c}\text { Decl. } \\
(\mathrm{J} 2000) \\
(4)\end{array}$ & $\begin{array}{c}\text { Net } \\
\text { Counts } \\
(5)\end{array}$ & $\begin{array}{l}\text { KS } \\
(6)\end{array}$ & $\begin{array}{c}\mathrm{E} \\
(\mathrm{keV}) \\
(7)\end{array}$ & $\begin{array}{c}\mathrm{N}_{\mathrm{H}} \\
\left(10^{22} \mathrm{~cm}^{-2}\right) \\
(8)\end{array}$ & $\begin{array}{c}\mathrm{kT} \\
(\mathrm{keV}) \\
(9)\end{array}$ & $\begin{array}{c}\mathrm{F}_{\mathrm{x}, \mathrm{abs}} \\
\left(\mathrm{erg} / \mathrm{cm}^{2} / \mathrm{s}\right) \\
(10)\end{array}$ & $\begin{array}{c}\log \mathrm{L}_{\mathrm{x}} \\
(\mathrm{erg} / \mathrm{s}) \\
(11)\end{array}$ & $\begin{array}{l}\text { Identification } \\
\text { (12) }\end{array}$ \\
\hline 245 & J054155.2-014847 & 54155.21 & -14847.4 & $15 \pm 6$ & 0.75 & 1.85 & $\ldots$ & $\ldots$ & $1.21 \mathrm{e}-15$ & $\ldots$ & 05415518-0148485 \\
\hline 246 & J054155.7-015508 & 54155.79 & -15508.5 & $135 \pm 13$ & 1.61 & 1.40 & $0.1_{-0.1}^{+0.2}$ & $1.4_{-0.7}^{+0.5}$ & $9.95 \mathrm{e}-15$ & 29.41 & HLL-99,05415579-0155092 \\
\hline 247 & J054156.5-014616 & 54156.57 & -14617.0 & $217 \pm 16$ & 0.82 & 2.11 & $0.6_{-0.2}^{+0.13}$ & $2.3_{-0.7}^{+1.5}$ & $2.78 \mathrm{e}-14$ & 30.17 & $05415656-0146168$ \\
\hline 248 & J054156.6-015552 & 54156.68 & -15552.4 & $170 \pm 14$ & 3.12 & 1.78 & $0.5_{-0.2}^{+0.3}$ & $1.5_{-0.5}^{+0.5}$ & $1.56 \mathrm{e}-14$ & 29.78 & HLL-110,05415668-0155526 \\
\hline 249 & J054156.7-015853 & 54156.74 & -15853.4 & $28 \pm 6$ & 0.49 & 2.29 & $\ldots$ & $\ldots$ & $4.33 \mathrm{e}-15$ & $\ldots$ & 05415674-0158538 \\
\hline 250 & J054156.7-015351 & 54156.79 & -15351.8 & $264 \pm 17$ & 1.92 & 2.77 & $1.7_{-0.4}^{+0.5}$ & $3.6_{-1.2}^{+2.2}$ & $1.18 \mathrm{e}-13$ & 30.71 & 05415680-0153521 \\
\hline 251 & J054157.0-015032 & 54157.02 & -15032.0 & $16 \pm 5$ & 1.47 & 1.86 & $\ldots$ & $\ldots$ & $1.61 \mathrm{e}-15$ & $\ldots$ & HLL-7,05415697-0150331 \\
\hline 252 & J054157.8-015127 & 54157.83 & -15127.7 & $38 \pm 7$ & 1.22 & 1.49 & $\ldots$ & $\ldots$ & $2.64 \mathrm{e}-15$ & $\ldots$ & HLL-14,05415786-0151278 \\
\hline 253 & J054158.0-015957 & 54158.10 & -15957.3 & $39 \pm 7$ & 1.82 & 1.37 & $\ldots$ & $\ldots$ & $3.09 \mathrm{e}-15$ & $\ldots$ & $05415807-0159575$ \\
\hline 254 & J054159.8-014735 & 54159.82 & -14735.3 & $39 \pm 8$ & 1.29 & 2.04 & $\ldots$ & $\ldots$ & $3.23 \mathrm{e}-15$ & $\ldots$ & 05415976-0147363 \\
\hline 255 & J054159.8-015949 & 54159.89 & -15949.6 & $7 \pm 4$ & 0.79 & 3.49 & $\ldots$ & $\ldots$ & $2.20 \mathrm{e}-15$ & $\ldots$ & 05415997-0159496 \\
\hline 256 & J054201.1-015621 & 54201.14 & -15621.3 & $23 \pm 6$ & 0.63 & 1.70 & $\ldots$ & $\ldots$ & $2.66 \mathrm{e}-15$ & $\ldots$ & 05420114-0156214 \\
\hline 257 & J054201.4-020008 & 54201.47 & -20008.3 & $15 \pm 5$ & 0.36 & 3.74 & $\ldots$ & $\ldots$ & $3.77 \mathrm{e}-15$ & $\ldots$ & $\ldots$ \\
\hline $258^{*}$ & J054201.7-015629 & 54201.71 & -15629.6 & $160 \pm 14$ & 1.84 & 2.29 & $0.9_{-0.3}^{+0.3}$ & $3.1_{-1.1}^{+2.4}$ & $2.19 \mathrm{e}-14$ & 29.94 & HLL-120,05420172-0156299 \\
\hline 259 & J054201.9-015629 & 54201.98 & -15629.2 & $19 \pm 5$ & 0.48 & 1.77 & $\ldots$ & $\ldots$ & $1.64 \mathrm{e}-15$ & $\ldots$ & 05420201-0156289 \\
\hline 260 & J054204.2-015201 & 54204.27 & -15201.8 & $16 \pm 5$ & 0.66 & 3.21 & $\ldots$ & $\ldots$ & $3.40 \mathrm{e}-15$ & $\ldots$ & $\ldots$ \\
\hline 261 & J054205.2-015344 & 54205.27 & -15344.4 & $65 \pm 9$ & 2.38 & 1.87 & $\ldots$ & $\ldots$ & $9.36 \mathrm{e}-15$ & $\ldots$ & 05420528-0153447 \\
\hline 262 & J054205.7-015928 & 54205.77 & -15928.7 & $42 \pm 8$ & 1.46 & 2.49 & $\ldots$ & $\ldots$ & $7.67 \mathrm{e}-15$ & $\ldots$ & 05420580-0159289 \\
\hline 263 & J054205.9-015252 & 54205.95 & -15252.0 & $136 \pm 13$ & 1.53 & 1.79 & $0.4_{-0.2}^{+0.2}$ & $2.2_{-0.6}^{+4.1}$ & $3.63 \mathrm{e}-14$ & 30.08 & HLL-35,05420597-0152522 \\
\hline 264 & J054207.5-015335 & 54207.51 & -15335.1 & $22 \pm 6$ & 0.52 & 1.75 & $\ldots$ & $\ldots$ & $1.68 \mathrm{e}-15$ & $\ldots$ & 05420744-0153368 \\
\hline 265 & J054207.5-015738 & 54207.55 & -15738.2 & $18 \pm 6$ & 0.72 & 3.66 & $\ldots$ & $\ldots$ & $6.05 \mathrm{e}-15$ & $\ldots$ & $\ldots$ \\
\hline 266 & J054207.6-015322 & 54207.60 & -15322.1 & $9 \pm 4$ & 0.52 & 3.05 & $\ldots$ & $\ldots$ & $1.73 \mathrm{e}-15$ & $\ldots$ & $\ldots$ \\
\hline 267 & J054208.9-015741 & 54208.98 & -15741.2 & $25 \pm 6$ & 0.77 & 2.83 & $\ldots$ & $\ldots$ & $4.68 \mathrm{e}-15$ & $\ldots$ & $\ldots$ \\
\hline 268 & J054209.3-015804 & 54209.35 & -15804.5 & $528 \pm 24$ & 1.37 & 1.78 & $0.5_{-0.2}^{+0.1}$ & $2.0_{-0.4}^{+0.7}$ & $5.32 \mathrm{e}-14$ & 30.27 & 05420934-0158048 \\
\hline $269^{*}$ & J054209.5-020004 & 54209.57 & -20004.2 & $1207 \pm 36$ & 4.47 & 1.95 & $0.6_{-0.1}^{+0.1}$ & $2.4_{-0.3}^{+0.4}$ & $1.36 \mathrm{e}-13$ & 30.69 & 05420956-0200044 \\
\hline 270 & J054210.2-020116 & 54210.22 & -20116.3 & $10 \pm 5$ & 0.72 & 2.74 & $\ldots$ & $\ldots$ & $1.25 \mathrm{e}-15$ & $\ldots$ & $\ldots$ \\
\hline 271 & J054211.0-014626 & 54211.07 & -14626.2 & $24 \pm 6$ & 0.76 & 2.09 & $\ldots$ & $\ldots$ & $2.99 \mathrm{e}-15$ & $\ldots$ & 05421113-0146255 \\
\hline $272^{*}$ & J054211.2-015943 & 54211.25 & -15943.3 & $1352 \pm 38$ & 1.53 & 1.26 & $0.2_{-0.1}^{+0.2}$ & $\ldots$ & $1.18 \mathrm{e}-13$ & $\ldots$ & 05421125-0159437 \\
\hline 273 & J054211.8-015637 & 54211.89 & -15637.9 & $9 \pm 4$ & 0.98 & 2.86 & $\ldots$ & $\ldots$ & $1.78 \mathrm{e}-15$ & $\ldots$ & $\ldots$ \\
\hline $274^{*}$ & J054213.1-015833 & 54213.19 & -15833.3 & $1251 \pm 36$ & 2.85 & 2.07 & $0.7_{-0.2}^{+0.2}$ & $2.5_{-0.3}^{+0.4}$ & $1.72 \mathrm{e}-13$ & 30.81 & 05421319-0158336 \\
\hline 275 & J054214.1-015141 & 54214.12 & -15141.3 & $13 \pm 6$ & 0.53 & 4.17 & $\ldots$ & $\ldots$ & $6.90 \mathrm{e}-15$ & $\ldots$ & $\ldots$ \\
\hline 276 & J054215.5-015500 & 54215.55 & -15500.1 & $15 \pm 5$ & 0.96 & 3.07 & $\ldots$ & $\ldots$ & $3.03 \mathrm{e}-15$ & $\ldots$ & $\ldots$ \\
\hline 277 & J054216.2-015614 & 54216.24 & -15614.6 & $180 \pm 15$ & 1.45 & 1.40 & $0.2_{-0.2}^{+0.2}$ & $0.8_{-0.2}^{+0.3}$ & $1.44 \mathrm{e}-14$ & 29.80 & Haro 5-53,05421620-0156148 \\
\hline 278 & J054216.7-015308 & 54216.79 & -15308.9 & $19 \pm 6$ & 0.99 & 2.10 & $\ldots$ & $\ldots$ & $2.80 \mathrm{e}-15$ & $\ldots$ & 05421677-0153091 \\
\hline 279 & J054218.0-015127 & 54218.08 & -15127.9 & $20 \pm 6$ & 0.77 & 2.78 & $\ldots$ & $\ldots$ & $4.74 \mathrm{e}-15$ & $\ldots$ & $\ldots$ \\
\hline
\end{tabular}


Table 1-Continued

\begin{tabular}{|c|c|c|c|c|c|c|c|c|c|c|c|}
\hline $\begin{array}{l}\text { No. } \\
\text { (1) }\end{array}$ & $\begin{array}{c}\text { Name } \\
(2)\end{array}$ & $\begin{array}{c}\text { R.A. } \\
\text { (J2000) } \\
(3)\end{array}$ & $\begin{array}{c}\text { Decl. } \\
(\mathrm{J} 2000) \\
(4)\end{array}$ & $\begin{array}{c}\text { Net } \\
\text { Counts } \\
(5)\end{array}$ & $\begin{array}{l}\text { KS } \\
\text { (6) }\end{array}$ & $\begin{array}{c}\mathrm{E} \\
(\mathrm{keV}) \\
(7)\end{array}$ & $\begin{array}{c}\left(10^{22} \mathrm{~cm}^{-2}\right) \\
(8)\end{array}$ & $\begin{array}{c}\mathrm{kT} \\
(\mathrm{keV}) \\
(9)\end{array}$ & $\begin{array}{c}\mathrm{F}_{\mathrm{x}, \mathrm{abs}} \\
\left(\mathrm{erg} / \mathrm{cm}^{2} / \mathrm{s}\right) \\
(10)\end{array}$ & $\begin{array}{c}\log L_{x} \\
(\mathrm{erg} / \mathrm{s}) \\
(11)\end{array}$ & $\begin{array}{l}\text { Identification } \\
\qquad(12)\end{array}$ \\
\hline 280 & J054218.4-015731 & 54218.45 & -15731.7 & $77 \pm 10$ & 0.72 & 3.39 & $\ldots$ & $\ldots$ & $2.31 \mathrm{e}-14$ & $\ldots$ & $\ldots$ \\
\hline 281 & J054218.6-015421 & 54218.63 & -15421.8 & $16 \pm 6$ & 0.60 & 3.24 & $\ldots$ & $\ldots$ & $5.04 \mathrm{e}-15$ & $\ldots$ & $\ldots$ \\
\hline 282 & J054221.2-015910 & 54221.29 & -15910.2 & $88 \pm 11$ & 0.82 & 1.64 & $0.0_{-\ldots}^{+0.1}$ & $1.0_{-0.2}^{+0.3}$ & $1.01 \mathrm{e}-14$ & 29.75 & 05422123-0159104 \\
\hline 283 & J054226.5-015913 & 54226.53 & -15913.2 & $8 \pm 5$ & 1.44 & 2.63 & & $\ldots$ & $2.19 \mathrm{e}-15$ & $\ldots$ & \\
\hline
\end{tabular}

a Col. (1) running source number; an asterisk indicates that additional source notes follow below; an $f$ inidicates that a flare or other obvious variability is seen in the source light curve, (2) J2000 IAU source name, (3)-(4) J2000 Chandra position with astrometric correction applied, (5) net source counts (= total counts - background counts) \pm net counts uncertainty (Gehrels 1986) in the $0.5-7.0 \mathrm{keV}$ range; source counts were measured in PSF-corrected $95 \%$ encircled energy regions except for the brightest sources where $99 \%$ encircled energy regions were used; smaller regions were used for some closely-spaced sources, (6) KS variability statistic, sources with KS $>1.7$ are variable (see text), (7) mean photon energy as determined from source events in the $0.5-7$ keV range, (8)-(9) absorption column density $\mathrm{N}_{H}$ and time-averaged characteristic temperature kT for sources with $\geq 90$ counts as determined from spectral fits with $1 \mathrm{~T}$ VAPEC optically thin plasma model using a fixed iron abundance $\mathrm{Fe}=0.3$ solar (errors are $90 \%$ confidence), (10) absorbed flux (0.5 - 7 keV) as determined from source event list (see text), (11) unabsorbed luminosity $(0.5-7 \mathrm{keV})$ at $\mathrm{d}=415 \mathrm{pc}$ determined from spectral fits of brighter sources where an estimate of $\mathrm{N}_{\mathrm{H}}$ and $\mathrm{kT}$ is available, (12) candidate IR or optical identification from 2MASS, SIMBAD, and B89 = Barnes et al. 1989; B = Beck et al. 2003; HLL = Haisch, Lada \& Lada 2000; HLP = Haisch et al. 2001, M = Meyer 1996. IR identifications found in the all-sky release of the 2MASS Point Source Catalog are listed by their coordinates (e.g. 05411324-0153306). Radio identifications (V-xx) are from $3.6 \mathrm{~cm}$ VLA observations of Rodriguez et al. 2003. 
5. $1 \mathrm{~T}$ model gives a high but uncertain temperature $\mathrm{kT} \geq 4 \mathrm{keV}$.

15 . HLL-131 is a likely IR counterpart, but there is a $\approx 4^{\prime \prime}$ positional offset even after correcting for systematic differences between CXO and HLL coordinates.

16. HLP-54 is a likely IR counterpart, but there is a $\approx 4^{\prime \prime}$ positional offset between CXO and HLP coordinates.

18. Flare spectrum gives $\mathrm{kT}=5.4_{-2.2}^{+\ldots} \mathrm{keV}$ and $\log \mathrm{Lx}=31.58 \mathrm{ergs} \mathrm{s}^{-1}$ (unabsorbed).

27. Flare spectrum gives $\mathrm{kT}=4.2_{-0.7}^{+0.6} \mathrm{keV}$ and $\log \mathrm{Lx}=30.99 \mathrm{ergs} \mathrm{s}^{-1}$ (unabsorbed). (Fig. 7)

36. Variability is present throughout the observation. Complex or rapidly varying temperature structure suspected.

39. Source lies in CCD gap. Flux is uncertain.

41. Weak flare whose spectrum gives $\mathrm{kT}=2.6_{-0.5}^{+1.0} \mathrm{keV}$ and $\log \mathrm{Lx}=30.80 \mathrm{ergs} \mathrm{s}^{-1}$ (unabsorbed).

52 . Weak flare. Insufficient counts to accurately determine flare parameters.

75. $1 \mathrm{~T}$ models give $\chi_{\text {red }}^{2}=1.09$ but $2 \mathrm{~T}$ models give a slightly better fit with $\chi_{\text {red }}^{2}=1.05$ and converge to $\mathrm{N}_{\mathrm{H}}$ $=1.7_{-0.2}^{+0.2} \times 10^{22} \mathrm{~cm}^{-2}, \mathrm{kT}_{1}=0.6_{-0.2}^{+0.3} \mathrm{keV}, \mathrm{kT}_{2}=2.7_{-0.3}^{+0.4} \mathrm{keV}$, and $\log \mathrm{Lx}=31.50 \mathrm{ergs} \mathrm{s}^{-1}$ (unabsorbed) . 81. Flare spectrum gives $\mathrm{kT}=9.8_{-4.5}^{+\ldots} \mathrm{keV}$ and $\log \mathrm{Lx}=31.08 \mathrm{ergs} \mathrm{s}^{-1}$ (unabsorbed). (Fig. 7)

85. Flare spectrum gives $\mathrm{kT}=3.8_{-0.5}^{+0.5} \mathrm{keV}$ and $\log \mathrm{Lx}=31.34 \mathrm{ergs} \mathrm{s}^{-1}$ (unabsorbed). (Figs. $7 \&$ 10)

93. Flare spectrum gives $\mathrm{kT}=3.5_{-1.1}^{+2.1} \mathrm{keV}$ and $\log \mathrm{Lx}=30.98 \mathrm{ergs} \mathrm{s}^{-1}$ (unabsorbed).

94. Slow variability is present throughout the observation. DEM model shows a double-peaked structure with cool and hot components.

99. $1 \mathrm{~T}$ models give $\chi_{\text {red }}^{2}=0.94$ but $2 \mathrm{~T}$ models give a slightly better fit with $\chi_{\text {red }}^{2}=0.92$ and converge to a larger absorption $\mathrm{N}_{H}=2.7_{-0.6}^{+0.4} \times 10^{22} \mathrm{~cm}^{-2}$.

104. Declining count rate may indicate decay phase of a flare.

105. Weak flare. Insufficient counts to accurately determine flare parameters.

108. Flare spectrum gives same temperature $\mathrm{kT}=3.3_{-0.6}^{+1.0} \mathrm{keV}$ and $\log \mathrm{Lx}=31.01 \mathrm{ergs} \mathrm{s}^{-1}$ (unabsorbed).

114. Declining count rate may indicate decay phase of a flare. Possible spectral contamination from nearby source.

120. Source may be affected by moderate pileup $(\approx 10-15 \%)$ during short periods when the count rate fluctuated to levels of $\approx 0.15 \mathrm{c} \mathrm{s}^{-1}$.

123. Count rate increased throughout the observation (Fig. 7).

133. $1 \mathrm{~T}$ models are only marginally acceptable with $\chi_{\text {red }}^{2}=1.35$. DEM model peaks near $1.7 \mathrm{keV}$ but shows substantial emission up to $\sim 4 \mathrm{keV}$. Declining count rate may indicate decay phase of a flare.

139. Slowly rising variability and outburst spectrum gives $\mathrm{kT}=4.2_{-1.6}^{+\ldots} \mathrm{keV}$ and $\log \mathrm{Lx}=30.79 \mathrm{ergs} \mathrm{s}^{-1}$ (unabsorbed).

141. The spectrum used to determine $\mathrm{N}_{\mathrm{H}}, \mathrm{kT}$ excludes data from a weak flare that occurred during the last $5 \mathrm{ksec}$ of the observation.

145. No reliable spectral fit because of contamination from nearby source no. 148. Possible optical counterpart is GSC 04771-00589.

148. No reliable spectral fit because of contamination from nearby source no. 145 .

152. $1 \mathrm{~T}$ models are only marginally acceptable with $\chi_{\text {red }}^{2}=1.38$. DEM model peaks near $1.4 \mathrm{keV}$.

153. $1 \mathrm{~T}$ models are not acceptable with $\chi_{\text {red }}^{2}=1.91$ but $2 \mathrm{~T}$ models are acceptable with $\chi_{\text {red }}^{2}=1.00 . \mathrm{N}_{\mathrm{H}}$ is from $2 \mathrm{~T}$ model $\left(\mathrm{kT}_{1}=0.8 \mathrm{keV}, \mathrm{kT}_{2}=3.4 \mathrm{keV}\right)$.

160. Weak flare. Insufficient counts to accurately determine flare parameters.

174. Flare spectrum gives $\mathrm{kT}=4.5_{-0.7}^{+0.6} \mathrm{keV}$ and $\log \mathrm{Lx}=31.45 \mathrm{ergs} \mathrm{s}^{-1}$ (unabsorbed). Moderate pileup may have occurred near flare peak. (Fig. 7)

175. Long duration flare with a secondary peak during decay phase (Fig. 7). Flare spectrum gives $\mathrm{kT}=$ $5.2_{-1.3}^{+2.6} \mathrm{keV}$ and $\log \mathrm{Lx}=31.47 \operatorname{ergs~s}^{-1}$ (unabsorbed). 
182. The counterpart name IRS 2b is from Nisini et al. (1994). Spectral fit parameters are uncertain because of possible contamination from source 183 located 1.7" to the north (Fig. 2). Some improvement is obtained with $2 \mathrm{~T}$ models which place most of the emission measure in a cool component at $\mathrm{kT}_{1} \approx 0.2 \mathrm{keV}$ and require a larger absorption column $\mathrm{N}_{H}=5.5_{-2.0}^{+2.5} \times 10^{22} \mathrm{~cm}^{-2}$.

183. Weak flare. Insufficient counts to accurately determine flare parameters. Spectral fit parameters are uncertain due to possible contamination from source 182 .

187. $1 \mathrm{~T}$ models give $\chi_{\text {red }}^{2}=1.10$ but $2 \mathrm{~T}$ models give a slightly better fit with $\chi_{\text {red }}^{2}=0.88$ and converge to a higher absorption $\mathrm{N}_{H}=6.1_{-2.7}^{+4.9} \times 10^{22} \mathrm{~cm}^{-2}, \mathrm{kT}_{1}=0.6_{-0.3}^{+1.0} \mathrm{keV}$, and a hotter component with uncertain temperature $\mathrm{kT}_{2} \geq 3 \mathrm{keV}$.

188. Weak flare. Flare spectrum gives $\mathrm{kT}=3.2_{-0.6}^{+0.8} \mathrm{keV}$ and same $\log \mathrm{Lx}=31.25 \mathrm{ergs} \mathrm{s}^{-1}$ (unabsorbed). 189. $1 \mathrm{~T}$ models are not acceptable $\left(\chi_{\text {red }}^{2}=1.52\right)$ nor are $2 \mathrm{~T}$ models $\left(\chi_{\text {red }}^{2}=1.68\right)$. Hard heavily-absorbed spectrum with uncertain temperature.

192. Slowly rising outburst (Fig. 7) with high absorption. The X-ray temperature shortly after onset reached values of at least $\mathrm{kT} \sim 6 \mathrm{keV}$, and possibly as high as $\mathrm{kT} \sim 10 \mathrm{keV}$. The unabsorbed luminosity varied from a pre-outburst value $\log \mathrm{Lx}=30.5 \mathrm{ergs} \mathrm{s}^{-1}$ to at least $\log \mathrm{Lx}=31.8 \mathrm{ergs} \mathrm{s}^{-1}$ during the outburst. Source is affected by moderate pileup $(\approx 27 \%)$ near end of observation.

193. Declining light curve may indicate decay phase of a flare.

196. $1 \mathrm{~T}$ models give a high but uncertain temperature $\mathrm{kT} \geq 3.1 \mathrm{keV}$.

207. Slowly rising outburst (Fig. 7). Very hard source. Time-averaged outburst spectrum gives $\mathrm{kT}=7.8_{-3.3}^{+\ldots}$ $\mathrm{keV}$ and $\log \mathrm{Lx}=31.4 \mathrm{ergs} \mathrm{s}^{-1}$ (unabsorbed). Temperature peaked about 4 hours after onset followed by a general decline, but there is evidence for at least one reheating event. DEM model of outburst spectrum suggests that most of the emission measure comes from plasma above $\sim 4 \mathrm{keV}$. Insufficient counts to analyze pre-outburst spectrum.

210. Declining light curve during first $\sim 20 \mathrm{ksec}$ of observation may indicate decay phase of a flare. Decay spectrum gives $\mathrm{kT}=4.9_{-1.1}^{+1.7} \mathrm{keV}$ and $\log \mathrm{Lx}=31.59 \mathrm{ergs} \mathrm{s}^{-1}$ (unabsorbed). Source may be affected by moderate pileup $(\approx 16 \%)$ at beginning of observation.

222. Weak flare. Insufficient counts to accurately determine flare parameters.

223. A faint IR source is visible at the X-ray position in 2MASS H and K-band images (2MASS J054150680153063), but is flagged as a filter glint artifact. Because of the close positional agreement with Chandra, the IR source flagged as an artifact may be real.

232. $1 \mathrm{~T}$ models are only marginally acceptable with $\chi_{\text {red }}^{2}=1.39$.

234. Declining light curve may indicate decay phase of a flare.

258. Possible double source.

269. Count rate declined throughout the observation (Fig. 7).

272. Soft source (low absorption). $1 \mathrm{~T}$ models underestimate the flux below $1 \mathrm{keV}$ and are not acceptable $\left(\chi_{\text {red }}^{2}=3.2\right)$. $2 \mathrm{~T}$ models give improved fits when the Fe abundance is allowed to vary $\left(\chi_{\text {red }}^{2}=1.24\right)$, converging to $\mathrm{Fe}=0.8$ solar with $\mathrm{kT}_{1}=0.77 \mathrm{keV}$ and $\mathrm{kT}_{2}=1.9 \mathrm{keV}$. DEM model peaks near $0.7 \mathrm{keV}$.

274. $1 \mathrm{~T}$ models are only marginally acceptable with $\chi_{\text {red }}^{2}=1.42$. DEM model peaks near $2 \mathrm{keV}$. Variability is present throughout the observation. 